\title{
Achievable rate region based on coset codes for multiple access channel with states
}

\author{
Arun Padakandla and S. Sandeep Pradhan, Member, IEEE
}

\begin{abstract}
We prove that the ensemble the nested coset codes built on finite fields achieves the capacity of arbitrary discrete memoryless point-to-point channels. Exploiting it's algebraic structure, we develop a coding technique for communication over general discrete multiple access channel with channel state information distributed at the transmitters. We build an algebraic coding framework for this problem using the ensemble of Abelian group codes and thereby derive a new achievable rate region. We identify non-additive and non-symmteric examples for which the proposed achievable rate region is strictly larger than the one achievable using random unstructured codes.
\end{abstract}

\section{INTRODUCTION}

The most common technique of proving achievability of rate regions in information theory is random coding 1 Traditionally, the distribution induced on the ensemble of codes is such that individual codewords are mutually independent. Furthermore, in communication models with multiple terminals, codebooks associated with these terminals are mutually independent of each other. Such an analysis has proved sufficient for single user and particular multi-terminal communication problems.2

The problem of distributed reconstruction of modulo- 2 sum of binary correlated sources studied by Körner and Marton [3] proved to be the first exception. As against to partitioning the source codes independently and uniformly into bins, they propose partitioning using cosets of a common linear code,

Arun Padakandla and S. Sandeep Pradhan are with the Department of Electrical and Computer Engineering, University of Michigan, Ann Arbor 48109-2122, USA.

This work was supported by NSF grant CCF-1116021.

${ }^{1}$ The other known techniques are based on Feinstein's lemma [1] and graph decomposition [2].

${ }^{2}$ However, characterization of optimal performance in many multi-terminal communication problems such as distributed source coding, interference channel, broadcast channel, multiple description coding remain open. 
thus building dependency across codebooks and codewords. Crucially exploiting the property of closure under addition of these cosets, they analyze a coding technique that strictly outperforms the best known strategy based on independent unstructured codes. Recently, a similar phenomenon has been identified by Philosof and Zamir [4] for a particular example of multiple access channel with state information distributed at the transmitters (MAC-DSTx). Restricting their attention to a binary symmetric noiseless additive doubly dirty MAC-DSTx (BDD-MAC), they propose a partition of the two channel codes into bins using cosets of a common linear code $\mathrm{S}^{3}$ They propose a coding technique, henceforth referred to as PZ-technique, that achieves the capacity of BDD-MAC and thereby prove strict sub-optimality of the best known coding technique based on independent unstructured codes. This is in contrast to point-topoint channels with state information at the transmitter (PTP-STx) where unstructured codes achieve the capacity [5].

Nevertheless ingenious, PZ-technique [4] is very specific to the additive and symmetric nature of the BDD-MAC studied therein. This technique being strictly more efficient than the currently known best strategy based on independent unstructured codes raises the following question. Is there a general coding framework for communicating over an arbitrary discrete MAC-DSTx, that reduces to the PZ-technique for the BDD-MAC, and that would yield an achievable rate region strictly larger than the best known achievable rate region using unstructured independent codes even for non-additive and non-symmetric MAC-DSTx?

In this article, we propose an algebraic framework for communication over an arbitrary MAC-DSTx and thereby answer the above questions in the affirmative. Our first step is to generalize the ensemble of linear codes employed in [4]. It is well known that linear codes do not achieve the capacity of point-topoint channels with or without state information available at the transmitter. They achieve only the mutual information of the channel with uniform input distribution. We propose, in section $\mathrm{IV}-\mathrm{A}$ an ensemble of codes, called nested coset codes, composed of two linear codes with one of them being a subset of the other, and prove in section IV-B, that they achieve the capacity of arbitrary PTP-STx, which is the first main result of this article. Using nested coset codes we are able to induce non-uniform single-letter distributions on the input alphabet while retaining much of useful algebraic structure and thus match any input distribution to the channel $4^{4}$ Achieving the capacity of arbitrary PTP-STx relies on employing joint

\footnotetext{
${ }^{3}$ Recall that communicating over a channel with state information at transmitter involves binning of the codebooks of the two transmitters [5].

${ }^{4}$ The technique of Gallager [6] involving a non linear mapping preceded by a linear code does not preserve the algebraic structure of the code.
} 
typical encoding and decoding. The foundation of our framework is therefore a study of codes endowed with an algebraic structure, nested coset codes in this case, using typical set encoding and decoding.

We present our coding scheme for MAC-DSTx in three pedagogical stages. We begin by identifying two key elements of PZ-technique 1) decoding mod-2 sum, instead of the pair of codewords chosen by the two transmitters and 2) choosing the bins of each user's code to be cosets of a common linear code to enable containment of the range of this mod-2 sum. The first stage, presented in section VI-B, captures all of the nontrivial elements of our framework in it's simplest setting. In this stage we employ nested coset codes built on finite fields, to decode the sum of codewords. The analysis of this technique enables us to derive a new achievable rate region for MAC-DSTx. The key elements of the first stage are (i) the use of nested coset codes to induce non-uniform input distributions, (ii) the use of joint typical encoding and decoding that enables us to analyze the probability of error over an arbitrary MAC-DSTx that is not constrained to be additive or symmetric, and (iii) an analysis of decoding of the sum of the pair of transmitted codewords chosen from two dependent codebooks. Indeed, the analysis of joint typical encoding and decoding of correlated codebooks with statistically dependent codewords involves several new elements. The reader is encouraged to peruse these in the proof of theorem 4

The significance of the rate region proved achievable in the first stage is illustrated through examples in section $\mathrm{VI-C}$ In particular, we provide an example for which it is necessary to induce non-uniform input distributions and is more efficient to decode the sum of transmitted codewords. We also randomly perturb the BDD-MAC and demonstrate that coding framework proposed herein can outperform unstructured independent codes. The channels being non-additive, it is significantly harder to provide analytical comparisons, and hence we resort to direct computation of rate regions achievable using unstructured independent and nested coset codes. These examples illustrate that structured-code based strategies do not hinge on the channel being additive but would benefit as long as the optimizing test channel from the auxiliary inputs to the channel output is not far from additive.

Does the rate region proved achievable using nested coset codes subsume the largest known achievable

${ }^{5}$ The coding technique proposed in the first stage reduces to that proposed in [4] for BDD-MAC and moreover Philosof and Zamir have proved strict sub-optimality of unstructured independent coding for BDD-MAC. This in itself establishes significance of theorem 4 Notwithstanding this, it is easy to argue significance of our generalization by appealing to continuity. An additive channel can be perturbed slightly to result in a non-additive channel for which the technique proposed in [4] may not be applicable as is. By continuity of the rate regions as a function of the channel parameters, one can see why the proposed coding scheme must perform strictly better than unstructured independent coding. Example 2 presented in section VI-C corroborates this. 
rate region using unstructured independent codes? It is our belief that strategies based on structured codes are not in lieu of their counterparts based on unstructured codes. In most cases, structured codes enable efficient decoding of a compressive' ${ }^{6}$ function of the two codewords. However, for decoding both the codewords, it turns out the strategy of using a common linear code to effect partition of the two codebooks is not optimal, instead one has to employ two independent linear codes. The rate region achieved using the latter strategy is equivalent to that achieved using unstructured independent codes 7 This leads us to the second stage of our coding scheme which is presented in section VII. Following the approach of Ahlswede and Han [9, Section VI], we glue together structured and unstructured coding techniques to derive an achievable rate region for communicating over a MAC-DSTx that combines structured and unstructured coding techniques. We present an example to illustrate how the gluing of unstructured and structured coding techniques can yield a rate region larger than either one, and their union. We remark that in spite of our inability to compute the achievable rate region proposed in section VII. we are able to demonstrate the significance of the same through an example.

If the channel is far from additive, it may not be efficient to decode the sum, with respect to a finite field, of codewords. For example, if the MAC-DSTx is doubly dirty with field addition replaced by addition of an Abelian group, referred to as group addition or group sum, then it is natural to decode group sum of codewords. In other words, the technique of decoding sum of codewords must be generalized to decoding any arbitrary bivariate function of the auxiliary inputs. In the third stage of our coding scheme, presented in section VIII, we consider decoding the group sum of the codewords. Specifically, codebooks are built over Abelian group alphabets and each encoder is provided with codebooks that possess a certain group structure. Analogous to the first stage, we propose joint typical encoding and decoding of group codes. Though essential elements of this analysis are similar to that of decoding sum of codewords chosen from nested coset codes over an arbitrary MAC-DSTx, the algebraic structure of a Abelian group being looser, leads to several new elements.

The importance of (i) decoding an appropriate bivariate function of codewords, and (ii) endowing codebooks with the appropriate algebraic structure is illustrated through an example discussed in section VIII Specifically, we indicate using numerical computation that for a quaternary doubly dirty MAC-

\footnotetext{
${ }^{6} f\left(U_{1}, U_{2}\right)$ is 'compressive' if $H\left(f\left(U_{1}, U_{2}\right)\right)$ is significantly lower than $H\left(U_{1}, U_{2}\right)$.

${ }^{7}$ Indeed, for the problem of distributed reconstruction of modulo-2 sum of binary sources, Körner Marton strategy [3] based on common linear codes is outperformed by Slepian-Wolf [7] strategy (or equivalently the strategy of Csiszár based on independent linear codes [8].) for the class of source distributions for which the modulo-2 sum is not sufficiently compressive. More precisely, if $H(X \oplus Y)>\frac{H(X, Y)}{2}$, then it is better to reconstruct $X \oplus Y$ using the technique of Slepian-Wolf or Csiszár.
} 
DSTx (QDD-MAC) wherein the operation is mod-4 addition, decoding mod-4 sum, which is the group operation in the quaternary alphabet, of the codewords strictly outperforms both independent unstructured and nested coset codes based strategies. In fact, significant gains for this problem are achievable using Abelian group codes. The reader is encouraged to peruse details in section VIII.

Several findings in the context of multi-terminal communication problems point to efficient strategies based on structured codes. Nazer and Gastpar [10] propose a strategy based on linear codes for computing the sum of sources over additive multiple access channels that outperforms earlier known strategies. Building on this technique, we develop a framework for computing sum of sources over an arbitrary multiple access channel in [11]. Sridharan et. al. [12] propose a coding technique based on lattices for communicating over a $K$-user Gaussian interference channel $(K \geq 3)$ that outperforms a natural extension of Han-Kobayashi technique [13] under the Gaussian input distribution. We propose an analogous coding technique based on nested linear codes [14] for the general discrete 3 -user interference channel and identify an example for which the proposed technique outperforms the natural extension of Han-Kobayashi technique [13]. Krithivasan and Pradhan [15] propose a framework based on structured codes for the distributed source coding problem that outperforms the best known strategy based on unstructured independent codes due to Berger and Tung [16]. We have employed the same ensemble of nested coset codes to strictly enlarge the largest known achievable rate region ${ }^{8}$ for the general $3-$ user discrete broadcast channel in [18].

We summarize by stating the significance of our contribution. Nested coset codes is currently the only ensemble of codes possessing an algebraic structure that has been proven to be optimal for general pointto-point channels. We employ the same to derive the largest known achievable rate region for a general discrete MAC-DSTx. Perhaps more importantly, our findings hint at a general theory of structured codes. Thus far, linear and nested linear codes have been employed to derive communication strategies for particular additive source and channel coding problems that outperform the best known techniques based on independent unstructured codes. Our findings indicate that strategies based on structured codes can be employed to analyze more intelligent encoding and decoding techniques for an arbitrary multi-terminal communication problem. This opens up the possibility of exploiting new degrees of freedom to enlarge achievable rate regions for many multi-terminal communication problems that have resisted a solution.

We begin with remarks on notation and state the form of typicality employed herein.

\footnotetext{
${ }^{8}$ This is obtained by a natural extension of Marton's [17] coding technique proposed for 2 -user broadcast channel.
} 


\section{Preliminaries: Notation AND Typicality}

\section{A. Notation}

We employ notation that is now widely employed in information theory literature supplemented by the following.

- We let $\mathbb{N}, \mathbb{R}$ denote the set of natural numbers and real numbers respectively. Calligraphic letters such as $\mathcal{X}, \mathcal{Y}$ are employed exclusively to denote finite sets. $\mathcal{F}_{q}$ denotes the finite field of cardinality $q$. For any set $A, \operatorname{cl}(A), \operatorname{cocl}(A)$ denote closure of $A$ and closure of the convex hull of $A$ respectively. If $A$ is a finite set, $|A|$ denotes cardinality of $A$.

- For positive integers $i \leq j,[i: j]:=\{i, i+1, \cdots, j\}$. We let $[j]:=[1: j]$.

- While + denotes addition in $\mathbb{R}$, we let $\oplus$ denote addition in a finite field. The particular finite field, which is uniquely determined (up to an isomorphism) by it's cardinality, is clear from context. When ambiguous, or to enhance clarity, we specify addition in $\mathcal{F}_{q}$ using $\oplus_{q}$. For $a, b \in \mathcal{F}_{q}, a \ominus b$ : $=a \oplus(-b)$, where $(-b)$ is the additive inverse of $b$.

- If $f: \mathcal{U} \rightarrow \mathcal{X}$ is a map, the $n$-letter extension of $f$ denoted $f^{n}: \mathcal{U}^{n} \rightarrow \mathcal{X}^{n}$ is defined $f^{n}\left(u^{n}\right):=$ $\left(f\left(u_{i}\right): i=1,2, \cdots, n\right)$.

- We employ the standard notation for probability mass functions (pmf). For example, if $p_{U X S Y}$ is a pmf on $\mathcal{U} \times \mathcal{X} \times \mathcal{S} \times \mathcal{Y}$, then $p_{U Y}$ is the corresponding marginal on $\mathcal{U} \times \mathcal{Y} \cdot p_{U Y}^{n}$ is the pmf on $\mathcal{U}^{n} \times \mathcal{Y}^{n}$ obtained as an $n$-fold product of $p_{U Y}$ i.e., $p_{U Y}^{n}\left(u^{n}, y^{n}\right)=\prod_{i=1}^{n} p_{U Y}\left(u_{i}, y_{i}\right)$. We write $U \sim p_{U}$ if $p_{U}$ is the pmf of $U$.

- The log and exp functions are taken with respect to base 2 .

- For $a \in \mathbb{N}, \pi(a):=\min \{k \in \mathbb{N}: k \geq a, k$ is a prime power $\}$.

- For a pmf $p_{U X S Y}$ defined on $\mathcal{U} \times \mathcal{X} \times \mathcal{S} \times \mathcal{Y}$, let

$$
\mathcal{R}\left(p_{U X S Y}, U\right):=\left\{u \in \mathcal{U}: \exists(x, s, y) \in \mathcal{X} \times \mathcal{S} \times \mathcal{Y}: p_{U X S Y}(u, x, s, y)>0\right\}
$$

denote the essential range of $U$. When clear from context, we omit the underlying pmf and let $\mathcal{R}(U)$ denote $\mathcal{R}\left(p_{U X S Y}, U\right)$.

\section{B. Typicality}

We adopt a slightly modified form [19] of the notion of robust typicality as proposed by Orlitsky and Roche [20] and adopted in [21]. In the sequel, we provide definitions and state the results employed in this article, in their simplest form. Since the following results have been well documented in books such as [22], [21], [23] among others, we omit proofs, and allude to one of the above references for the same. 
Let $\mathcal{X}_{1}, \mathcal{X}_{2}$ be finite sets and $X:=\left(X_{1}, X_{2}\right)$ be a pair of random variables taking values in $\mathcal{X}:=\mathcal{X}_{1} \times$ $\mathcal{X}_{2}$ with pmf $p_{X}:=p_{X_{1} X_{2}}$. Let $X^{n}:=\left(X_{1}^{n}, X_{2}^{n}\right)$ be $n$ independent and identically distributed copies of $X$. For a pair $a=\left(a_{1}, a_{2}\right) \in \mathcal{X}$, and an $n$-tuple $x^{n}:=\left(x_{1}^{n}, x_{2}^{n}\right) \in \mathcal{X}^{n}$, let $N\left(a \mid x^{n}\right)=\sum_{i=1}^{n} 1_{\left\{\left(x_{1 i}, x_{2 i}\right)=a\right\}}$ be the number of occurrences of $a$ in $x^{n}$. Lastly, for $j \in\{1,2\}$, let $j \in\{1,2\} \backslash\{j\}$ denote the element in it's complement. We are now set to define typical set. For any $\delta>0$, let

$$
T_{\delta}:=\left\{x^{n} \in \mathcal{X}^{n}:\left|\frac{N\left(a \mid x^{n}\right)}{n}-p_{X}(a)\right| \leq \frac{\delta p_{X}(a)}{\log |\mathcal{X}|} \text { for all } a \in \mathcal{X}\right\}
$$

be the typical set on $\mathcal{X}$ with respect to $\operatorname{pmf} p_{X}$ and parameter $\delta>0$. For $j=1,2$, the projection

$$
T_{\delta}\left(X_{j}\right):=\left\{x_{j}^{n} \in \mathcal{X}_{j}^{n}: \text { there exists } x_{\dot{j}}^{n} \in \mathcal{X}_{\dot{j}}^{n} \text { such that }\left(x_{1}^{n}, x_{2}^{n}\right) \in T_{\delta}\right\}
$$

is the typical set on $\mathcal{X}_{j}$ with respect to $\operatorname{pmf} p_{X}$ and parameter $\delta>0$. For $j=1,2$ and any $x_{\dot{j}}^{n} \in \mathcal{X}_{\dot{j}}^{n}$,

$$
T_{\delta}\left(X_{j} \mid x_{j}^{n}\right):=\left\{x_{j}^{n} \in \mathcal{X}_{j}^{n} \text { such that }\left(x_{1}^{n}, x_{2}^{n}\right) \in T_{\delta}\right\}
$$

is the typical set on $\mathcal{X}_{j}$ conditioned on $x_{\dot{f}}^{n}$ with respect to distribution $p_{X}$ and parameter $\delta>0$. Before we state the basic results, the following remark is worth noting.

Remark 1: If for any $a \in \mathcal{X}, p_{X}(a)=0$, and $x^{n} \in T_{\delta}$, then $N\left(a \mid x^{n}\right)=0$.

Lemma 1: If $x^{n} \in T_{\delta}$, then for every $n \in \mathbb{N},\left|\frac{1}{n} \log p_{X^{n}}\left(x^{n}\right)+H(X)\right| \leq \delta,\left|\frac{1}{n} \log p_{X_{j}^{n}}\left(x_{j}^{n}\right)+H\left(X_{j}\right)\right| \leq$ $\delta$ for $j \in[2]$ and therefore $\left|\frac{1}{n} \log p_{X_{j}^{n} \mid X_{\dot{f}}^{n}}\left(x_{j}^{n} \mid x_{\dot{f}}^{n}\right)+H\left(X_{j} \mid X_{\dot{f}}\right)\right| \leq 2 \delta$.

Lemma 2: For every $\epsilon>0, \delta>0$, there exists $N(\epsilon, \delta) \in \mathbb{N}$, such that for every $n \geq N(\epsilon, \delta)$, $\operatorname{Pr}\left(X^{n} \in T_{\delta}\right) \geq 1-\epsilon$, and therefore, $\operatorname{Pr}\left(X_{j}^{n} \in T_{\delta}\left(X_{j}\right)\right) \geq 1-\epsilon$, for each $j \in[2]$. Moreover,

$$
\operatorname{Pr}\left(X^{n} \notin T_{\delta}\right) \leq \exp \left\{-n \lambda \delta^{2}\right\}, \text { where } \lambda=\frac{1}{(\log |\mathcal{X}|)^{2}} \min _{a \in \mathcal{X}}\left\{p_{X}^{2}(a): a \in \mathcal{X}, p_{X}(a)>0\right\}
$$

While the first statement of lemma 2 can be proved using Cheybyshev inequality, the second statement, due to Hoeffding [24], Sanov [25], requires a finer analysis. The reader is referred to [22, Problem 11 Pg 43] for an idea of the proof.

Lemma 3: For every $\delta>0$, there exists $N_{1}(\delta), N_{2}(\delta) \in \mathbb{N}$, such that,

1) for every $n \geq N_{1}(\delta)$, $\exp \{n(H(X)-2 \delta)\} \leq\left|T_{\delta}\right| \leq \exp \{n(H(X)+2 \delta)\}$, and

2) for every $n \geq N_{2}(\delta), \exp \left\{n\left(H\left(X_{j}\right)-2 \delta\right)\right\} \leq\left|T_{\delta}\left(X_{j}\right)\right| \leq \exp \left\{n\left(H\left(X_{j}\right)+2 \delta\right)\right\}$.

Lemma 4: For every $\epsilon>0, \delta>0$, there exists $N(\epsilon, \delta) \in \mathbb{N}$, such that for every $n \geq N(\epsilon, \delta), x_{\dot{j}}^{n} \in$ $T_{\delta}\left(X_{\dot{f}}\right)$, implies $\operatorname{Pr}\left(X_{j}^{n} \in T_{2 \delta}\left(X_{j} \mid x_{\dot{j}}^{n}\right) \mid X_{\dot{f}}^{n}=x_{\dot{j}}^{n}\right) \geq 1-\epsilon$ and therefore $\operatorname{Pr}\left(X_{\dot{f}} \in T_{\delta}\left(X_{\dot{f}}\right), X^{n} \notin T_{2 \delta}\right) \leq \epsilon$.

Lemma 5: For every $\delta>0$, there exists $N(\delta) \in \mathbb{N}$, such that, for every $n \geq N(\delta), x_{j}^{n} \in T_{\delta}\left(X_{\dot{j}}\right)$ we have $\exp \left\{n\left(H\left(X_{j} \mid X_{\dot{f}}\right)-3 \delta\right)\right\} \leq\left|T_{2 \delta}\left(X_{j} \mid x_{\dot{f}}^{n}\right)\right| \leq \exp \left\{n\left(H\left(X_{j} \mid X_{\dot{f}}\right)+3 \delta\right)\right\}$. 


\section{PTP-STX: DEFINITIONS AND CAPACITY}

We begin with a description of the PTP-STx [5] and relevant notions in section III-A. In section III-B, we state the capacity region of PTP-STx as derived by Gelfand and Pinsker.

\section{A. Definitions - PTP-STX, achievability and capacity}

Consider a point-to-point channel with knowledge of channel state at transmitter (PTP-STx) studied by Gelfand and Pinsker [5]. Let $\mathcal{X}$ and $\mathcal{Y}$ denote finite input and output alphabet sets respectively. Transition probabilities depend on a random parameter, called state, that takes values in a finite set $\mathcal{S}$. The discrete time channel is (i) time invariant, i.e., pmf of $Y_{i}$, the output at time $i$, conditioned on $\left(X_{i}, S_{i}\right)$, the input and state at time $i$, is invariant with $i$, (ii) memoryless, i.e., $Y_{i}$ is conditionally independent of $\left(X_{t}, S_{t}\right): 1 \leq t<i$ given $\left(X_{i}, S_{i}\right)$, and (iii) used without feedback, i.e., encoder has no knowledge of outputs observed by decoder. Let $W_{Y \mid X S}(y \mid x, s)$ be the probability of observing $y \in \mathcal{Y}$ at the output given $x \in \mathcal{X}$ is input to PTP-STx in state $s \in \mathcal{S}$. The state at time $i, S_{i}$ is (i) independent of $\left(X_{t}, S_{t}, Y_{t}\right): 1 \leq t<i$, and (ii) identically distributed for all $i$. Let $W_{S}(s)$ be probability of PTP-STx being in state $s \in \mathcal{S}$. We assume the sequence of states is non-causally available at the encoder. The input is constrained with respect to a cost function $\kappa: \mathcal{X} \times \mathcal{S} \rightarrow[0, \infty)$. We assume that the cost is time-invariant and additive i.e., cost of input $X^{n}$ to the channel in state $S^{n}$ is $\bar{\kappa}^{n}\left(X^{n}, S^{n}\right):=\frac{1}{n} \sum_{i=1}^{n} \kappa\left(X_{i}, S_{i}\right)$. We refer to this channel as PTP-STx $\left(\mathcal{S}, W_{S}, \mathcal{X}, \kappa, \mathcal{Y}, W_{Y \mid X S}\right)$.

Definition 1: A PTP-STx code $(n, \mathscr{M}, e, d)$ consists of (i) an index set $\mathcal{M}$ of messages, of cardinality $\mathscr{M}$, (ii) an encoder map $e: \mathcal{M} \times \mathcal{S}^{n} \rightarrow \mathcal{X}^{n}$, and (iii) a decoder map $d: \mathcal{Y}^{n} \rightarrow \mathcal{M}$.

Assuming a uniform pmf on the set of messages, we define the average error probability and the cost of a PTP-STx code.

Definition 2: The error probability of PTP-STx code $(n, \mathscr{M}, e, d)$ conditioned on message $m \in \mathcal{M}$ is

$$
\xi(e, d \mid m):=\sum_{s^{n} \in \mathcal{S}^{n}} \sum_{\substack{y^{n}: d\left(y^{n}\right) \\ \neq m}} W_{S^{n}}\left(s^{n}\right) W_{Y^{n} \mid X^{n}, S^{n}}\left(y^{n} \mid e\left(m, s^{n}\right), s^{n}\right) .
$$

The average error probability of PTP-STx code $(n, \mathscr{M}, e, d)$ is $\bar{\xi}(e, d):=\sum_{m=1}^{\mathscr{M}} \frac{1}{\mathscr{M}} \xi(e, d \mid m)$. The average cost of transmitting message $m \in \mathcal{M}$ is $\tau(e \mid m):=\sum_{s^{n} \in \mathcal{S}^{n}} W_{S^{n}}\left(s^{n}\right) \bar{\kappa}^{n}\left(e\left(m, s^{n}\right), s^{n}\right)$ and the average cost of PTP-STx code $(n, \mathscr{M}, e, d)$ is $\tau(e):=\frac{1}{\mathscr{M}} \sum_{m=1}^{\mathscr{M}} \tau(e \mid m)$.

Definition 3: A rate cost pair $(R, \tau) \in[0, \infty)^{2}$ is achievable if for every $\eta>0$, there exists $N(\eta) \in \mathbb{N}$ such that for all $n>N(\eta)$, there exists a PTP-STx code $\left(n, \mathscr{M}^{(n)}, e^{(n)}, d^{(n)}\right)$ such that (i) $\frac{\log \mathscr{M}^{(n)}}{n} \geq$ $R-\eta$, (ii) $\bar{\xi}\left(e^{(n)}, d^{(n)}\right) \leq \eta$, and (iii) average cost $\tau\left(e^{(n)}\right) \leq \tau+\eta$. The capacity region is $\mathbb{C}(\tau)$ : $=\operatorname{cl}\{R \geq 0:(R, \tau)$ is achievable $\}$. 
In a celebrated result, Gelfand and Pinsker [5] derived a single letter characterization of $\mathbb{C}(\tau)$. In the next section, we state this characterization.

\section{B. Capacity of PTP-STx}

Definition 4: Let $\overline{\mathbb{D}}(\tau)$ be the collection of pmfs $p_{V X S Y}$ on $\mathcal{V} \times \mathcal{X} \times \mathcal{S} \times \mathcal{Y}$ such that (i) $\mathcal{V}$ is a finite set, (ii) $p_{S}=W_{S}$, (iii) $p_{Y \mid X S V}=p_{Y \mid X S}=W_{Y \mid X S}$, (iv) $p_{X \mid S V}(x \mid s, v) \in\{0,1\}$ for all $(v, x, s) \in \mathcal{V} \times \mathcal{X} \times \mathcal{S}$ and (v) $\mathbb{E}\{\kappa(X, S)\} \leq \tau$. Let

$$
\mathbb{D}(\tau)=\left\{p_{V X S Y} \in \overline{\mathbb{D}}(\tau):\left|\mathcal{R}\left(p_{V X S Y}, V\right)\right| \leq \min \left\{(|\mathcal{X}| \cdot|\mathcal{S}|)^{2},(|\mathcal{X}|+|\mathcal{S}|+|\mathcal{Y}|-2) \cdot|\mathcal{X}| \cdot|\mathcal{S}|\right\}\right\} .
$$

For any pmf $p_{V X S Y}$ defined on $\mathcal{V} \times \mathcal{X} \times \mathcal{S} \times \mathcal{Y}$, let $\alpha\left(p_{V X S Y}\right):=[0, I(V ; Y)-I(V ; S)]$, and

$$
\bar{\alpha}(\tau):=\operatorname{cocl}\left(\bigcup_{p_{V X S Y} \in \overline{\mathbb{D}}(\tau)} \alpha\left(p_{V X S Y}\right)\right), \alpha(\tau):=\operatorname{cocl}\left(\bigcup_{p_{V X Y} \in \mathbb{D}(\tau)} \alpha\left(p_{V X S Y}\right)\right) .
$$

Theorem 1: $\mathbb{C}(\tau)=\alpha(\tau)=\bar{\alpha}(\tau)$.

Gelfand and Pinsker [5] proved theorem [1 for channels without a cost constraint. While the central elements of their proof can be adopted for cost constrained channels, the sufficiency of restricting to test channels $p_{V S X Y}$ satisfying condition (iv) in definition 4 is established in [26, Lemma 2], which is attributed to Cohen. A cardinality bound on $|\mathcal{V}|$ can be established using Fenchel-Eggleston strengthening of Carathéodory's theorem [21, Appendix C] as done in [18, Lemma 9]. In particular, one can first prove the upper bound $\min \{|\mathcal{X}| \cdot|\mathcal{S}|,|\mathcal{X}|+|\mathcal{S}|+|\mathcal{Y}|-2\}$ on $|\mathcal{V}|$ for test channels $p_{V S X Y}$ that do not satisfy condition (iv) in definition 4. Any such test channel $p_{V S X Y}$ can be mapped to a test channel $p_{\tilde{V} S X Y}$ that satisfies condition (iv) in definition 4 without compromising on the achievable rate for which $|\tilde{\mathcal{V}}| \leq|\mathcal{X}| \cdot|\mathcal{S}| \cdot|\mathcal{V}|$

\section{NESTED COSET CODES ACHIEVE CAPACITY OF POINT TO POINT CHANNELS}

\section{A. Nested coset PTP-STx codes}

Gelfand and Pinsker prove achievability of $\mathbb{C}(\tau)$ by averaging error probability over an ensemble of PTP-STx codes. A code in this ensemble is specified by a corresponding auxiliary code $\lambda_{O}$ built over an auxiliary set and a mapping. An ingenious technique of partitioning (binning) $\lambda_{O}$ into $\mathcal{M}$ bins, one for each message $m \in \mathcal{M}$, is the key feature of the coding technique. In the following, we consider PTP-STx codes which are endowed with a nested coset code structure. The distinguishing feature of a nested coset PTP-STx code is that $\lambda_{O}$ is a coset code built over a finite field $\mathcal{F}_{q}$ and $\lambda_{O}$ is partitioned 
into bins by cosets of a sub coset code $\lambda_{I} \subseteq \lambda_{O}$. In the sequel, we describe nested coset codes and define a nested coset PTP-STx code.

We begin with a brief review of coset and nested coset codes. An $(n, k)$ coset code is a collection of vectors in $\mathcal{F}_{q}^{n}$ obtained by adding a bias vector to a $k$-dimensional subspace of $\mathcal{F}_{q}^{n}$. If $\lambda_{O} \subseteq \mathcal{F}_{q}^{n}$ and $\lambda_{I} \subseteq \lambda_{O}$ are $(n, k+l)$ and $(n, k)$ coset codes respectively, then $q^{l}$ cosets $\lambda_{O} / \lambda_{I}$ that partition $\lambda_{O}$ is a nested coset code. We refer to this as nested coset code $\left(n, k, l, g_{I}, g_{O / I}, b^{n}\right)$ where $b^{n}$ is the bias vector, $g_{I} \in \mathcal{F}_{q}^{k \times n}$ and $g_{O}^{T}=\left[\begin{array}{ll}g_{I}^{T} & g_{O / I}^{T}\end{array}\right] \in \mathcal{F}_{q}^{(k+l) \times n}$ are generator matrices of $\lambda_{I}$ and $\lambda_{O}$ respectively.

An informed reader will begin to see the structure we are after. The bins are cosets of the smaller linear code $\lambda_{I}$. The entire collection of bins forms a coset of the larger linear code $\lambda_{O}$. The message to be sent to the decoder indexes the bins. For this nested coset code, we let $v^{n}\left(a^{k}, m^{l}\right):=a^{k} g_{I} \oplus m^{l} g_{O / I} \oplus b^{n}$ denote a generic codeword in coset $c\left(m^{l}\right):=\left\{v^{n}\left(a^{k}, m^{l}\right) \in \mathcal{F}_{q}^{n}: a^{k} \in \mathcal{F}_{q}^{k}\right\}$. We refer to $c\left(m^{l}\right)$ as the coset corresponding to message $m^{l}$. The following is therefore a natural characterization of a nested coset PTP-STx code.

Definition 5: A nested coset PTP-STx code $(n, \mathscr{M}, e, d)$ over $\mathcal{F}_{q}$ is a PTP-STx code if there exists (i) a nested coset code $\left(n, k, l, g_{I}, g_{O / I}, b^{n}\right)$ over $\mathcal{F}_{q}$, ii) map $f: \mathcal{F}_{q} \times \mathcal{S} \rightarrow \mathcal{X}$ and, (iii) a $1: 1$ map $\iota: \mathcal{M} \rightarrow \mathcal{F}_{q}^{l}$ such that $e\left(m, s^{n}\right) \in\left\{f^{n}\left(a^{k} g_{I} \oplus \iota(m) g_{O / I} \oplus b^{n}, s^{n}\right): a^{k} \in \mathcal{F}_{q}^{k}\right\}$.

\section{B. Achievability}

We now state and prove our first main finding - nested coset PTP-STx codes achieve $\mathbb{C}(\tau)$.

Theorem 2: For a PTP-STx $\left(\mathcal{S}, W_{S}, \mathcal{X}, \kappa, \mathcal{Y}, W_{Y \mid X S}\right)$, if $R \in \mathbb{C}(\tau)$, then there exists a sequence $\left(n, \mathscr{M}^{(n)}, e^{(n)}, d^{(n)}\right): n \geq 1$ of nested coset PTP-STx codes over $\mathcal{F}_{q}$ that achieves $(R, \tau)$, where $q=$ $\pi\left(\min \left\{(|\mathcal{X}| \cdot|\mathcal{S}|)^{2},(|\mathcal{X}|+|\mathcal{S}|+|\mathcal{Y}|-2) \cdot|\mathcal{X}| \cdot|\mathcal{S}|\right\}\right)$.

Proof: Consider any pmf $p_{V X S Y} \in \mathbb{D}(\tau)$ and $\eta>0$. We prove the existence of a nested coset PTPSTx code $\left(n, \mathscr{M}^{(n)}, e^{(n)}, d^{(n)}\right)$ of rate $\frac{\log \mathscr{M}^{(n)}}{n} \geq I(V ; Y)-I(V ; S)-\eta$, average cost $\tau\left(e^{(n)}\right) \leq \tau+\eta$ and average probability of error $\bar{\xi}\left(e^{(n)}, d^{n}\right) \leq \eta$ for every $n \in \mathbb{N}$ sufficiently large. The underlying finite field is of cardinality $\pi\left(\min \left\{(|\mathcal{X}| \cdot|\mathcal{S}|)^{2},(|\mathcal{X}|+|\mathcal{S}|+|\mathcal{Y}|-2) \cdot|\mathcal{X}| \cdot|\mathcal{S}|\right\}\right)$ referred to as $\pi$ for short.

We prove the existence by averaging the error probability over a specific ensemble of nested coset PTP-STx codes. We begin with a description of a generic code in this ensemble.

Consider a nested coset PTP-STx code $\left(n, k, l, g_{I}, g_{O / I}, b^{n}\right)$, denoted $\lambda_{O} / \lambda_{I}$ with parameters

$$
\begin{aligned}
k & :=\left\lceil n\left(1-\frac{H(V \mid S)}{\log \pi}+\frac{\eta}{8 \log \pi}\right)\right\rceil \\
l & :=\left\lfloor n\left(1-\frac{H(V \mid Y)}{\log \pi}-\frac{\eta}{8 \log \pi}\right)\right\rfloor-k .
\end{aligned}
$$


The reader is advised to bear in mind our notation is not reflective of $k$ and $l$ being functions of $n$. This abuse of notation reduces clutter. We specify encoding and decoding rules that map $\lambda_{O} / \lambda_{I}$ into a corresponding nested coset PTP-STx code.

The encoder is provided with nested coset code $\lambda_{O} / \lambda_{I}$. The message is used to index one among $\pi^{l}$ cosets of $\lambda_{O} / \lambda_{I}$. For simplicity, we assume that the set of messages $\mathcal{M}$ is $\mathcal{V}^{l}$, and $M^{l} \in \mathcal{V}^{l}$ to be the uniformly distributed random variable representing user's message. The encoder observes the state sequence $S^{n}$ and populates the list $L\left(M^{l}, S^{n}\right)=\left\{v\left(a^{k}, M^{l}\right):\left(v\left(a^{k}, M^{l}\right), S^{n}\right) \in T_{\frac{\delta}{2}}(V, S), a^{k} \in \mathcal{F}_{q}^{k}\right\}$ of codewords in the coset corresponding to the message that are jointly typical with the state sequence, where $\delta:=\frac{1}{2} \min \left\{\frac{\eta}{48}, \frac{\eta \log (|\mathcal{V}\|\mathcal{X}\| \mathcal{S}||\mathcal{Y}|)}{\kappa_{\max }}\right\}, \kappa_{\max }:=\max \{\kappa(x, s):(x, s) \in \mathcal{X} \times \mathcal{S}\}$. If $L\left(M^{l}, S^{n}\right)$ is empty, it picks a codeword uniformly at random from coset $c\left(M^{l}\right)$. Otherwise, it picks a codeword uniformly at random from $L\left(M^{l}, S^{n}\right)$. Let $V\left(A^{k}, M^{l}\right)$ denote the picked codeword in either case. The encoder computes $X^{n}\left(M^{l}, S^{n}\right):=f^{n}\left(V^{n}\left(A^{k}, M^{l}\right), S^{n}\right)$, where $f: \mathcal{V} \times \mathcal{S} \rightarrow \mathcal{X}$ is any map that satisfies $p_{X \mid V S}(f(v, s) \mid v, s)=1$ for all pairs $(v, s) \in \mathcal{V} \times \mathcal{S} . X^{n}\left(M^{l}, S^{n}\right)$ is fed as input to the channel.

The decoder observes the received vector $Y^{n}$ and populates the list

$$
D\left(Y^{n}\right):=\left\{m^{l} \in \mathcal{V}^{l}: \exists v^{n}\left(a^{k}, m^{l}\right) \text { such that }\left(v^{n}\left(a^{k}, m^{l}\right), Y^{n}\right) \in T_{\delta}(V, Y)\right\} .
$$

If $D\left(Y^{n}\right)$ is a singleton, the decoder declares the content of $D\left(Y^{n}\right)$ as the decoded message pair. Otherwise, it declares an error.

The above encoding and decoding rules map $\lambda_{O} / \lambda_{I}$ into a corresponding nested coset PTP-STx code $\left(n, \mathscr{M}^{n}, e^{(n)}, d^{(n)}\right)$ of rate $\frac{\log \mathscr{M}^{(n)}}{n}=\frac{l \log \pi}{n}$. Observe that, for $n \geq N_{1}(\eta):=\left\lceil\frac{8 \log \pi}{\eta}\right\rceil$, we have

$$
\begin{aligned}
n\left(1-\frac{H(V \mid S)}{\log \pi}+\frac{\eta}{8 \log \pi}\right) \leq k & \leq n\left(1-\frac{H(V \mid S)}{\log \pi}+\frac{\eta}{8 \log \pi}\right)+1 \\
& \leq n\left(1-\frac{H(V \mid S)}{\log \pi}+\frac{\eta}{4 \log \pi}\right),
\end{aligned}
$$

and similarly,

$$
\begin{aligned}
n\left(1-\frac{H(V \mid Y)}{\log \pi}-\frac{\eta}{8 \log \pi}\right) \geq k+l & \geq n\left(1-\frac{H(V \mid Y)}{\log \pi}-\frac{\eta}{8 \log \pi}\right)-1 \\
& \geq n\left(1-\frac{H(V \mid Y)}{\log \pi}-\frac{\eta}{4 \log \pi}\right) .
\end{aligned}
$$

Combining the upper bound for $k$ in (4) and the lower bound for $k+l$ in (6), we get

$$
\frac{l \log \pi}{n} \geq H(V \mid S)-H(V \mid Y)-\frac{\eta}{2}=I(V ; Y)-I(V ; S)-\frac{\eta}{2}
$$

Since $\lambda_{O} / \lambda_{I}$ was a generic nested coset code satisfying (1), 2, we have characterized, through our encoding and decoding maps, an ensemble of nested coset PTP-STx codes, one for each $n \in \mathbb{N}, n \geq N_{1}(\eta)$ 
of rate at least $I(V ; Y)-I(V ; S)-\frac{\eta}{2}$. It suffices to prove existence of a PTP-STx code $\left(n, \mathscr{M}^{(n)}, e^{(n)}, d^{(n)}\right)$ in this ensemble, one for each $n \in \mathbb{N}$ sufficiently large, with average probability of error $\xi\left(e^{(n)}, d^{(n)}\right) \leq \eta$ and average cost constraint $\tau\left(e^{(n)}\right) \leq \tau+\eta$. This is done by averaging $\xi\left(e^{(n)}, d^{(n)}\right)$ over the ensemble.

Consider a random nested coset code $\left(n, k, l, G_{I}, G_{O / I}, B^{n}\right)$, denoted $\Lambda_{O} / \Lambda_{I}$, with parameters $n, k, l$ satisfying [1] and 22. Let $G_{I} \in \mathcal{V}^{k \times n}, G_{O / I} \in \mathcal{V}^{l \times n}$ and bias vector $B^{n} \in \mathcal{V}^{n}$ be mutually independent and uniformly distributed on their respective range spaces. In the sequel, we study the average probability of error $\xi\left(e^{(n)}, d^{(n)}\right)$ of the corresponding random nested coset PTP-STx code. Towards this end, we begin with a few remarks on notation. Let $V^{n}\left(a^{k}, m^{l}\right):=a^{k} G_{I} \oplus m^{l} G_{O / I} \oplus B^{n}$ denote a generic codeword in coset $C\left(m^{l}\right):=\left\{V^{n}\left(a^{k}, m^{l}\right): a^{k} \in \mathcal{V}^{k}\right\}$ corresponding to message $m^{l}$.

In order to study $\xi\left(e^{(n)}, d^{(n)}\right)$, we need to characterize the error events associated with the random nested coset PTP-STx code corresponding to $\Lambda_{O} / \Lambda_{I}$. If $\epsilon_{1}:=\left\{S^{n} \notin T_{\frac{\delta}{4}}(S)\right\}, \epsilon_{2}:=\left\{\phi_{\frac{\delta}{2}}\left(S^{n}, M^{l}\right)=0\right\}$, where $\phi_{\frac{\delta}{2}}\left(s^{n}, m^{l}\right):=\sum_{a^{k} \in \mathcal{V}^{k}} 1_{\left\{\left(V^{n}\left(a^{k}, m^{l}\right), s^{n}\right) \in T_{\frac{\delta}{2}}^{n}(V S)\right\}}$, then the error event at the encoder is contained in $\epsilon_{1} \cup \epsilon_{2}$. The error event at the decoder is contained in $\epsilon_{3}^{c} \cup \epsilon_{4}$, where $\epsilon_{3}:=\cup_{a^{k} \in \mathcal{V}^{k}}\left\{\left(V^{n}\left(a^{k}, M^{l}\right), Y^{n}\right) \in\right.$ $\left.T_{\delta}^{n}(V, Y)\right\}$ and $\epsilon_{4}:=\cup_{\hat{m}^{l} \neq M^{l}} \cup_{a^{k} \in \mathcal{V}^{k}}\left\{\left(V^{n}\left(a^{k}, \hat{m}^{l}\right), Y^{n}\right) \in T_{\delta}^{n}(V, Y)\right\}$. It suffices to derive an upper bound on $P\left(\epsilon_{1}\right)+P\left(\epsilon_{1}^{c} \cap \epsilon_{2}\right)+P\left(\left(\epsilon_{1} \cup \epsilon_{2}\right)^{c} \cap \epsilon_{3}^{c}\right)+P\left(\epsilon_{4}\right)$. In the sequel, we derive an upper bound on each term of the above sum.

Lemma 2 guarantees the existence of $N_{2}(\eta) \in \mathbb{N}^{9}$ such that $\forall n \geq N_{2}(\eta), P\left(\epsilon_{1}\right) \leq \frac{\eta}{16}$. In appendix A. we prove the existence of $N_{3}(\eta) \in \mathbb{N}$, such that $\forall n \geq N_{3}(\eta)$,

$$
P\left(\epsilon_{1}^{c} \cap \epsilon_{2}\right) \leq \exp \left\{-n \log \pi\left(\frac{k}{n}-\left(1-\frac{H(V \mid S)}{\log \pi}+\frac{3 \delta}{4 \log \pi}\right)\right)\right\} .
$$

Substituting the lower bound in (3) for $k$ in (8), for all $n \geq \max \left\{N_{1}(\eta), N_{3}(\eta)\right\}$, we have

$$
P\left(\epsilon_{1}^{c} \cap \epsilon_{2}\right) \leq \exp \left\{-n\left(\frac{\eta}{8}-\frac{3 \delta}{4}\right)\right\} \leq \exp \left\{-n\left(\frac{7 \eta}{64}\right)\right\},
$$

where the last inequality follows from the choice of $\delta$.

We now consider $P\left(\left(\epsilon_{1} \cup \epsilon_{2}\right)^{c} \cap \epsilon_{3}^{c}\right)$. An informed reader will recognize that an upper bound on this term can be derived using a typical application of conditional frequency typicality lemma 4 . For the sake of completeness we state the arguments. The encoding rule ensures, $\left(\epsilon_{1} \cup \epsilon_{2}\right)^{c} \subseteq\left\{\left(V^{n}\left(M^{l}, S^{n}\right), S^{n}\right) \in\right.$

\footnotetext{
${ }^{9}$ Since $\delta$ is a function of $\eta$, the dependence of $N_{2}(\eta)$ on $\delta$ is captured through $\eta$.
} 
$\left.T_{\frac{\delta}{2}}^{n}(V, S)\right\}$, and thus

$$
\begin{aligned}
& P\left(\left(\epsilon_{1} \cup \epsilon_{2}\right)^{c} \cap \epsilon_{3}^{c}\right) \leq P\left(\left\{\left(V^{n}\left(M^{l}, S^{n}\right), S^{n}\right) \in T_{\frac{\delta}{4}}^{n}(V, S)\right\} \cap \epsilon_{3}^{c}\right) \\
& \quad \leq \sum_{\left(v^{n}, s^{n}\right) \in T_{\frac{\delta}{2}}^{n}(V, S)} P\left(\left(V^{n}\left(M^{l}, S^{n}\right), S^{n}\right)=\left(v^{n}, s^{n}\right)\right) P\left(\epsilon_{3}^{c} \mid\left(V^{n}\left(M^{l}, S^{n}\right), S^{n}\right)=\left(v^{n}, s^{n}\right)\right) \\
& \quad \leq \sum_{\left(v^{n}, s^{n}\right) \in T_{\frac{\delta}{2}}^{n}(V, S)} P\left(\left(V^{n}\left(M^{l}, S^{n}\right), S^{n}\right)=\left(v^{n}, s^{n}\right)\right) P\left(Y^{n} \notin T_{\delta}\left(Y \mid v^{n}, s^{n}\right) \mid\left(V^{n}\left(M^{l}, S^{n}\right), S^{n}\right)=\left(v^{n}, s^{n}\right)\right)(10)
\end{aligned}
$$

For any $\left(v^{n}, s^{n}\right) \in T_{\frac{\delta}{2}}^{n}(V, S)$, note that,

$$
P\left(\begin{array}{c}
Y^{n}=y^{n}, \\
X^{n}\left(M^{l}, S^{n}\right)=x^{n}
\end{array} \mid \begin{array}{c}
\left(V^{n}\left(M^{l}, S^{n}\right), S^{n}\right) \\
=\left(v^{n}, s^{n}\right)
\end{array}\right)=\prod_{i=1}^{n} P\left(X_{i}=x_{i}, Y_{i}=y_{i} \mid V_{i}=v_{i}, S_{i}=s_{i}\right)
$$

where the second equality follows from Markov chain $V-(X, S)-Y$. By lemma 4 , there exists $N_{4}(\eta) \in \mathbb{N}$ such that for all $n \geq N_{4}(\eta)$

$$
P\left(\left(Y^{n}, X^{n}\left(M^{l}, S^{n}\right)\right) \notin T_{\delta}^{n}\left(X, Y \mid v^{n}, s^{n}\right) \mid\left(V^{n}\left(M^{l}, S^{n}\right), S^{n}\right)=\left(v^{n}, s^{n}\right)\right) \leq \frac{\eta}{8} .
$$

Substituting (11) in 10 , we have $P\left(\left(\epsilon_{1} \cup \epsilon_{2}\right)^{c} \cap \epsilon_{3}\right) \leq \frac{\eta}{8}$ for all $n \geq N_{4}(\eta)$. It remains to provide an upper bound on $P\left(\epsilon_{4}\right)$. In appendix $\mathbb{B}$, we prove the existence of $N_{5}(\eta) \in \mathbb{N}$ such that $\forall n \geq N_{5}(\eta)$, $P\left(\left(\epsilon_{1} \cup \epsilon_{2} \cup \epsilon_{3}\right)^{c} \cap \epsilon_{4}\right) \leq \exp \left\{-n \log \pi\left(1-\frac{H(V \mid Y)}{\log \pi}-\frac{3 \delta}{2 \log \pi}-\frac{k+l}{n}\right)\right\}$. For $n \geq \max \left\{N_{1}(\eta), N_{5}(\eta)\right\}$, the upper bound for $k+l$ derived in $(6)$ is substituted to yield, $P\left(\left(\epsilon_{1} \cup \epsilon_{2} \cup \epsilon_{3}\right)^{c} \cap \epsilon_{4}\right) \leq \exp \left\{-n\left(\frac{\eta}{8}-\frac{3 \delta}{2}\right)\right\} \leq$ $\exp \left\{-n\left(\frac{3 \eta}{32}\right)\right\}$.

We have therefore proved that for every $n \geq \max \left\{N_{i}(\eta): i \in[5]\right\}$, there exists at least one nested coset PTP-STx code $\left(n, \pi^{l}, e, d\right)$ over $\mathcal{F}_{\pi}$ for which $\bar{\xi}(e, d) \leq \frac{\eta}{8}+\exp \left\{-n \frac{7 \eta}{64}\right\}+\frac{\eta}{8}+\exp \left\{-n \frac{3 \eta}{32}\right\}$. For $n \geq \max \left\{N_{i}(\eta): i \in[6]\right\}$, where $N_{6}(\eta)=\left\lceil\frac{32}{3 \eta} \log \frac{8}{\eta}\right\rceil, \bar{\xi}(e, d) \leq \frac{\eta}{2}$. It only remains to prove this code satisfies the average cost constraint. It can be verified that $\tau(e) \leq \frac{\eta}{2} \kappa_{\max }+\left(1-\frac{\eta}{2}\right)\left(\tau+\frac{\delta \kappa_{\max }}{2 \log (|\mathcal{X}||\mathcal{S}|)}\right)$. The choice of $\delta$ ensures that $\tau(e) \leq \frac{\eta}{2} \kappa_{\max }+\left(\tau+\frac{\eta}{2}\right)$. Since $\kappa_{\max } \in \mathbb{R}$ is bounded, this proves the existence of a sequence $\left(n, \pi^{l(n)}, e^{(n)}, d^{(n)}\right): n \geq 1$ of nested coset PTP-STx codes that achieve $(R, \tau)$ for every $R \in \mathbb{C}(\tau)$.

The codewords of $\Lambda_{O}$ being uniformly distributed over $\mathcal{F}_{\pi}^{n}$ (c.f. Lemma 6 (i)), the probability of it being jointly typical with a typical state sequence $s^{n}$ is $\frac{\left|T_{\delta}(U \mid S)\right|}{\pi^{n}}=\exp \{n(H(U \mid S)-\log \pi)\}$. This indicates that each coset must contain roughly $\frac{q^{n}}{\left|T_{\delta}(U \mid S)\right|}=\frac{q^{n}}{q^{n(H(U \mid S))}}=q^{n(\log \pi-H(U \mid S))}$ codewords. Indeed, it suffices to partition $\Lambda_{O}$ with a coset of rate $\frac{k}{n}>1-\frac{H(U \mid S)}{\log \pi} .1-\frac{H(U \mid S)}{\log \pi}$ being in general larger than $\frac{I(U ; S)}{\log \pi}$, we conclude that the constraint of linearity forces us to increase the rate of the binning code.

However, the sparsity of typical vectors in a random linear code comes to our rescue when we attempt to pack cosets. The decoder looks for all vectors in the auxiliary code that are jointly typical with the 
received vector $Y^{n}$. In unstructured random coding, since each codeword is individually typical with high probability, the rate of auxiliary code is bounded from above by $\frac{I(U ; Y)}{\log \pi}$. The typical vectors being sparse in random linear code, a similar argument as above enables us to enlarge the auxiliary code to a rate $1-\frac{H(U \mid Y)}{\log \pi}$. The rate of the code is thus $\left(1-\frac{H(U \mid Y)}{\log \pi}\right)-\left(1-\frac{H(U \mid S)}{\log \pi}\right)=\frac{I(U ; Y)-I(U ; S)}{\log \pi}$.

We have thus proved nested coset codes achieve the capacity of arbitrary PTP-STx. The interested reader is referred to [27] wherein nested lattice codes are proved to achieve capacity of arbitrary continuous point to point channels. In order to achieve capacity of arbitrary continuous PTP-STx, it is necessary to construct lattices which induce arbitrary test channels when employed for source quantization. In a related work, Gariby and Erez [28] construct lattices for source coding of continuous sources that yield a family of quantization error distributions.

\section{MAC-DSTX: DEFINITIONS, LARGEST KNOWN ACHIEVABLE RATE REGION}

The rest of the article is aimed at deriving achievable rate regions for the MAC-DSTx. In this section, we lay the necessary groundwork. In particular, we describe MAC-DSTx and precisely state relevant notions such as code, achievability in section $\mathrm{V}-\mathrm{A}$. In section $\mathrm{V}-\mathrm{B}$, we provide a characterization of the rate region based on independent unstructured codes. We illustrate this rate region for BDD-MAC in section $\mathrm{V}-\mathrm{C}$ and highlight the reasons for it's sub-optimality. This will set the stage for it's enlargement in subsequent sections.

\section{A. Definitions : MAC-DSTx, code and achievability}

Consider the two user multiple access analogue of PTP-STx [5]. Let $\mathcal{X}_{1}$ and $\mathcal{X}_{2}$ denote finite input alphabet sets and $\mathcal{Y}$, the output alphabet set. Transition probabilities depend on a random vector parameter $S:=\left(S_{1}, S_{2}\right)$, called state, that takes values in a finite set $\mathcal{S}:=\mathcal{S}_{1} \times \mathcal{S}_{2}$. The discrete time channel is (i) time invariant, i.e., pmf of $Y_{i}$, the output at time $i$, conditioned on inputs $\boldsymbol{X}_{\boldsymbol{i}}:=\left(X_{1 i}, X_{2 i}\right)$ and state $\mathbf{S}_{\mathbf{i}}:=\left(S_{1 i}, S_{2 i}\right)$ at time $i$, is invariant with $i$, (ii) memoryless, i.e., $Y_{i}$ is conditionally independent of $\left(\boldsymbol{X}_{\boldsymbol{t}}, \boldsymbol{S}_{\boldsymbol{t}}\right): 1 \leq t<i$ given $\boldsymbol{X}_{\boldsymbol{i}}, \boldsymbol{S}_{\boldsymbol{i}}$, and (iii) used without feedback. Let $W_{Y \mid \boldsymbol{X} \boldsymbol{S}}(y \mid \boldsymbol{x}, \boldsymbol{s})$ be the probability of observing $y \in \mathcal{Y}$ at the output given $\boldsymbol{x}:=\left(x_{1}, x_{2}\right) \in \mathcal{X}:=\mathcal{X}_{1} \times \mathcal{X}_{2}$ is input to the channel in state $s:=\left(s_{1}, s_{2}\right) \in \mathcal{S}$. The state at time $i, \boldsymbol{S}_{\boldsymbol{i}}$ is (i) independent of $\left(\boldsymbol{S}_{\boldsymbol{t}}, \boldsymbol{X}_{\boldsymbol{t}}, Y_{t}\right): 1 \leq t<i$, and (ii) identically distributed for all $i$. Let $W_{\boldsymbol{S}}(\boldsymbol{s})$ be the probability of MAC-DSTx being in state $s \in \mathcal{S}$. We assume $S_{j}^{n}$ is non-causally known to encoder $j$. Input $X_{j}$ is constrained with respect to a cost function $\kappa_{j}: \mathcal{X}_{j} \times \mathcal{S}_{j} \rightarrow[0, \infty)$. We assume that the cost is time-invariant and additive i.e., cost of input $X_{j}^{n}$ at input $j$ to the channel in state $S^{n}$ is $\bar{\kappa}_{j}^{n}\left(X_{j}^{n}, S_{j}^{n}\right):=\frac{1}{n} \sum_{i=1}^{n} \kappa_{j}\left(X_{j i}, S_{j i}\right)$. We refer to this channel 
as MAC-DSTx $\left(\mathcal{S}, W_{\boldsymbol{S}}, \mathcal{X}, \kappa, \mathcal{Y}, W_{Y \mid \boldsymbol{X}, \boldsymbol{S}}\right)$. Towards characterizing a new inner bound for the capacity region of a MAC-DSTx, we begin with definitions of relevant notions such as achievability and capacity.

Definition 6: A MAC-DSTx code $\left(n, \mathscr{M}_{1}, \mathscr{M}_{2}, e_{1}, e_{2}, d\right)$ consists of (i) index sets $\mathcal{M}_{j}$ of messages, of cardinality $\mathscr{M}_{j}$ for $j=1,2$ (ii) encoder maps $e_{j}: \mathcal{M}_{j} \times \mathcal{S}_{j}^{n} \rightarrow \mathcal{X}_{j}^{n}$ for $j=1,2$, and (iii) a decoder $\operatorname{map} d: \mathcal{Y}^{n} \rightarrow \mathcal{M}_{1} \times \mathcal{M}_{2}$.

We let $\mathscr{M}:=\left(\mathscr{M}_{1}, \mathscr{M}_{2}\right), \mathbf{e}:=\left(e_{1}, e_{2}\right)$ and refer to above as MAC-DSTx code $(n, \mathscr{M}, \mathbf{e}, d)$. Assuming the pair of messages to be uniformly distributed, we define the average error probability and the cost of a MAC-DSTx code as follows.

Definition 7: The average error probability of MAC-DSTx code $(n, \mathscr{M}, \mathbf{e}, d)$ conditioned on message $\boldsymbol{m}:=\left(m_{1}, m_{2}\right) \in \mathcal{M}:=\mathcal{M}_{1} \times \mathcal{M}_{2}$ is

$$
\xi(\mathbf{e}, d \mid \boldsymbol{m}):=\sum_{\boldsymbol{s}^{n} \in \mathcal{S}^{n}} W_{\boldsymbol{S}^{n}}\left(\boldsymbol{s}^{n}\right) \sum_{y^{n}: d\left(y^{n}\right) \neq \boldsymbol{m}} W_{Y^{n} \mid \boldsymbol{X}^{n}, \boldsymbol{S}^{n}}\left(y^{n} \mid e_{1}\left(m_{1}, s_{1}^{n}\right), e_{2}\left(m_{2}, s_{2}^{n}\right), \boldsymbol{s}^{n}\right) .
$$

The average error probability is $\bar{\xi}(\mathbf{e}, d):=\sum_{\boldsymbol{m} \in \mathcal{M}} \frac{1}{\mathscr{M}_{1} \mathscr{M}_{2}} \xi(\mathbf{e}, d \mid \boldsymbol{m})$. The average cost of transmitting message pair $\boldsymbol{m}$ is $\tau(\mathbf{e} \mid \boldsymbol{m}):=\left(\tau_{1}\left(e_{1} \mid m_{1}\right), \tau_{2}\left(e_{2} \mid m_{2}\right)\right)$, where

$$
\tau_{j}\left(e_{j} \mid m_{j}\right):=\sum_{s_{j}^{n} \in \mathcal{S}_{j}^{n}} W_{S_{j}^{n}}\left(s_{j}^{n}\right) \bar{\kappa}_{j}^{n}\left(e_{j}\left(m_{j}, s_{j}^{n}\right), s_{j}^{n}\right) .
$$

The average cost of the code is $\tau(\mathbf{e}):=\sum_{\boldsymbol{m} \in \mathcal{M}} \frac{1}{\mathscr{M}_{1} \mathscr{M}_{2}} \tau(\mathbf{e} \mid \boldsymbol{m})$, where $\tau(\mathbf{e})=\left(\tau\left(e_{1}\right), \tau\left(e_{2}\right)\right)$.

Definition 8: A rate cost quadruple $(\mathbf{R}, \boldsymbol{\tau}) \in[0, \infty)^{4}$ is achievable if for every $\eta>0$, there exists $N(\eta) \in \mathbb{N}$ such that for all $n>N(\eta)$, there exists a MAC-DSTx code $\left(n, \mathcal{M}^{(n)}, \mathbf{e}^{(n)}, d^{(n)}\right)$ such that

(i) $\frac{\log \mathcal{M}_{j}^{(n)}}{n} \geq R_{j}-\eta$ for $j=1,2$, (ii) $\bar{\xi}\left(\mathbf{e}^{(n)}, d^{(n)}\right) \leq \eta$, and (iii) $\tau_{j}\left(e_{j}^{(n)}\right) \leq \tau_{j}+\eta$, for $j=1,2$. The capacity region $\mathbb{C}(\boldsymbol{\tau}):=\operatorname{cocl}\left(\left\{\mathbf{R} \in[0, \infty)^{2}:(\mathbf{R}, \boldsymbol{\tau})\right.\right.$ is achievable $\left.\}\right)$.

The coding technique that achieves capacity of PTP-STx [5] can be generalized to obtain an achievable rate region for MAC-DSTx. For a general MAC-DSTx this is the largest known inner bound to $\mathbb{C}(\boldsymbol{\tau})$. We provide a characterization of the same in the following section.

\section{B. Largest known achievable rate region using unstructured codes}

Definition 9: Let $\mathbb{D}(\boldsymbol{\tau})$ be collection of pmfs $p_{\boldsymbol{U} \boldsymbol{X} \boldsymbol{S} Y}$ on $\mathcal{U}^{2} \times \mathcal{X} \times \mathcal{S} \times \mathcal{Y}$, where $\boldsymbol{U}$ denotes $U_{1}, U_{2}$ and $\mathcal{U}^{2}$ is a two fold Cartesian product of a finite set $\mathcal{U}$, such that (i) $p_{\boldsymbol{S}}=W_{\boldsymbol{S}}$, (ii) $p_{Y \mid \boldsymbol{X} S \boldsymbol{U}}=p_{Y \mid \boldsymbol{X} \boldsymbol{S}}=$ $W_{Y \mid \boldsymbol{X} S}$, (iii) $p_{U_{j} \mid S U_{\mathfrak{f}}}=p_{U_{j} \mid \boldsymbol{S}}=p_{U_{j} \mid S_{j}}$ and $p_{X_{j} \mid S \boldsymbol{U} X_{\text {f }}}=p_{X_{j} \mid \boldsymbol{S} \boldsymbol{U}}=p_{X_{j} \mid S_{j} U_{j}}$ for any distinct elements $j, j \in\{1,2\}$, (iv) $p_{X_{j} \mid S_{j} U_{j}}\left(x_{j} \mid s_{j}, u_{j}\right) \in\{0,1\}$ for all $\left(u_{j}, s_{j}, x_{j}\right), j=1,2$ and (v) $\mathbb{E}\left\{\kappa_{j}\left(X_{j}, S_{j}\right)\right\} \leq \tau_{j}$ 
for $j=1,2$. For $p_{\boldsymbol{U} \boldsymbol{X} \boldsymbol{S} Y} \in \mathbb{D}(\boldsymbol{\tau})$, let $\alpha\left(p_{\boldsymbol{U} \boldsymbol{X} \boldsymbol{S} Y}\right)$ be defined as the set

$$
\left\{\begin{array}{cl}
\left(R_{1}, R_{2}\right) \in[0, \infty)^{2}: \quad & R_{1} \leq I\left(U_{1} ; Y U_{2}\right)-I\left(U_{1} ; S_{1}\right), R_{2} \leq I\left(U_{2} ; Y U_{1}\right)-I\left(U_{2} ; S_{2}\right), \\
& R_{1}+R_{2} \leq I(\boldsymbol{U} ; Y)+I\left(U_{1} ; U_{2}\right)-\sum_{j=1}^{2} I\left(U_{j} ; S_{j}\right)
\end{array}\right\}
$$

and

$$
\alpha(\boldsymbol{\tau}):=\operatorname{cocl}\left(\bigcup_{p_{U X S Y} \in \mathbb{D}(\tau)} \alpha\left(p_{\boldsymbol{U} \boldsymbol{X} S Y}\right)\right) .
$$

Theorem 3: $\alpha(\boldsymbol{\tau}) \subseteq \mathbb{C}(\boldsymbol{\tau})$.

Achievability of $\alpha\left(p_{\boldsymbol{U} X \boldsymbol{S} Y}\right)$ can be proved by employing the encoding technique proposed by Gelfand and Pinsker [5] at each encoder and joint decoding proposed by Ahlswede [29], Liao [30]. In the sequel, we provide an illustration of this coding technique for BDD-MAC.

\section{Rate region achievable using unstructured codes for BDD-MAC}

Philosof and Zamir characterize $\mathbb{C}(\boldsymbol{\tau})$ for BDD-MAC using PZ-technique and prove $\alpha(\boldsymbol{\tau}) \subsetneq \mathbb{C}(\boldsymbol{\tau})$ for the same. In order to identify the key elements of PZ-technique, we briefly analyze unstructured coding (this section), PZ-technique (section VI-A and set the stage for a new coding scheme.

BDD-MAC is a MAC-DSTx with binary alphabets $\mathcal{S}_{j}=\mathcal{X}_{j}=\mathcal{Y}=\{0,1\}, j=1,2$. The state sequences are independent Bernoulli- $\frac{1}{2}$ processes, i.e., $W_{\boldsymbol{S}}(\boldsymbol{s})=\frac{1}{4}$ for all $s \in \mathcal{S}$. The channel transition is described by the relation $Y=X_{1} \oplus_{2} S_{1} \oplus_{2} X_{2} \oplus_{2} S_{2}$. An additive Hamming cost is assumed on the input, i.e., $\kappa_{j}\left(1, s_{j}\right)=1$ and $\kappa_{j}\left(0, s_{j}\right)=0$ for any $s_{j} \in \mathcal{S}_{j}, j=1,2$ and the input is subject to a symmetric cost constraint $\boldsymbol{\tau}=(\tau, \tau)$.

We describe the test channel $p_{\boldsymbol{U} \boldsymbol{S} \boldsymbol{X} Y} \in \mathbb{D}(\boldsymbol{\tau})$ that achieves $\alpha(\boldsymbol{\tau})$. For each user $j$, consider the test channel that achieves the Gelfand-Pinsker capacity treating the other user as noise i.e., $p_{U_{j} S_{j} X_{j}}(0,1,1)=$ $p_{U_{j} S_{j} X_{k}}(1,0,1)=\frac{\tau}{2}, p_{U_{j} S_{j} X_{j}}(0,0,0)=p_{U_{j} S_{j} X_{j}}(1,1,0)=\frac{1-\tau}{2}$. Philosof and Zamir prove $p_{\boldsymbol{U} \boldsymbol{S} \boldsymbol{X}}=$ $p_{U_{1} S_{1} X_{1}} p_{U_{2} S_{2} X_{2}}$ achieves $\alpha(\boldsymbol{\tau})=\left\{\mathbf{R}: R_{1}+R_{2} \leq\left|2 h_{b}(\tau)-1\right|^{+}\right\}$, where $|\cdot|^{+}$denotes upper convex envelope.

Let us take a closer look at achievability of the vertex $\left(2 h_{b}(\tau)-1,0\right)$ using the above test channel. Since user 2 has no message to transmit, it picks a single bin with roughly $2^{n I\left(U_{2} ; S_{2}\right)}=2^{n\left(1-h_{b}(\tau)\right)}$ codewords independently and uniformly from the entire space of binary vectors. User 1 picks $2^{n R_{1}}$ bins each with roughly $2^{n I\left(U_{1} ; S_{1}\right)}=2^{n\left(1-h_{b}(\tau)\right)}$ independently and uniformly distributed binary vectors. Encoder 2 observes $S_{2}^{n}$ and chooses a codeword, say $U_{2}^{n}$, that is within a Hamming distance of roughly $n \tau$ from $S_{2}^{n}$ and transmits $X_{2}^{n}=U_{2}^{n} \oplus_{2} S_{2}^{n}$. Encoder 1 performs a similar encoding, except that it restricts the choice of $U_{1}^{n}$ to the bin indexed by user 1's message, and transmits $X_{1}^{n}=U_{1}^{n} \oplus_{2} S_{1}^{n}$. 
What is the maximum rate $R_{1}$ at which user 1 can transmit it's message? Decoder receives $Y^{n}=$ $U_{1}^{n} \oplus_{2} U_{2}^{n}$ and looks for all pairs of codewords that are jointly typical with $Y^{n}$. Since any pair of binary $n$-length vectors are jointly typical ( $U_{1}$ and $U_{2}$ are independent and uniform), the decoding rule reduces to finding all pairs of binary $n$-length vectors in the pair of codebooks that sum to the received vector $Y^{n}$. All bins chosen independently without structure imply that any bin of user 1's codebook when added to the user 2's codebook (a single bin) results in roughly $2^{n\left(2-2 h_{b}(\tau)\right)}$ distinct vectors. Therefore, we cannot hope to pack more than roughly $\frac{2^{n}}{2^{n\left(2-2 h_{b}(q)\right)}}=2^{n\left(2 h_{b}(q)-1\right)}$ bins in user 1's codebook. We remark that an explosion in the range of sum of transmitted codewords severely limits achievable rate.

We make a few observations. Effectively, communication occurs over the $\left(U_{1}, U_{2}\right)-Y$ channel and the test channel induces the Markov chain $\left(U_{1}, U_{2}\right)-U_{1} \oplus_{2} U_{2}-Y$. It would therefore be more efficient to communicate information over the $U_{1} \oplus_{2} U_{2}-Y$ channel which suggests an efficient utilization of $U_{1} \oplus_{2} U_{2}$-space. Having chosen codewords in each bin independently and moreover the two users' bins independently, each message pair utilizes $2^{n\left(2-2 h_{b}(\tau)\right)}$ vectors in the $U_{1} \oplus_{2} U_{2}$-space. In section VI-A, we summarize PZ-technique, wherein the algebraic structure in the codebooks is exploited for more efficient utilization of $U_{1} \oplus_{2} U_{2}$-space.

\section{AN ACHIEVABLE RATE REgION USING NESTED COSET CODES}

\section{A. Nested linear codes for BDD-MAC}

We present PZ-technique proposed for BDD-MAC. The encoding and decoding techniques are similar to that stated in $\mathrm{V}-\mathrm{C}$ except for one key difference. The bins of user 1 and 2's codebooks are cosets of a common linear code. In particular, let $\lambda_{I}$ denote a linear code of rate roughly equal to $1-h_{b}(\tau)$ that can quantize a uniform source, state $S_{j}^{n}$ in our case, within an average Hamming distortion of $\tau$. Since user 2 has no message to transmit, it employs $\lambda_{I}$ as it's only bin. Encoder 1 employs $2^{n R_{1}}$ cosets of $\lambda_{I}$ within a larger linear code, called $\lambda_{O}$, as it's bins. Note that rate of $\lambda_{O}$ is roughly $R_{1}+1-h_{b}(\tau)$. Encoding rule is as described in section $\mathrm{V}-\mathrm{C}$.

The codebook of user 2 when added to any bin of user 1's code results in a coset of $\lambda_{I}$, and therefore contains approximately at most $2^{n\left(1-h_{b}(\tau)\right)}$ codewords. Moreover, since $U_{1}^{n}$ lies in $\lambda_{I}$, user 2's codeword $U_{2}^{n}$ and the received vector $Y^{n}=U_{1}^{n} \oplus_{2} U_{2}^{n}$ lie in the same coset ${ }^{10}$ Since the channel is noiseless, user 1 may employ all cosets of $\lambda_{I}$ and therefore communicate at rate $h_{b}(\tau)$ which is larger than $2 h_{b}(\tau)-1$ for all $\tau \in\left(0, \frac{1}{2}\right)$.

\footnotetext{
${ }^{10}$ This is also because the channel is noiseless.
} 
Let us identify key elements of PZ-technique. Each message pair corresponds to roughly $2^{n\left(1-h_{b}(\tau)\right)}$ vectors in $U_{1} \oplus_{2} U_{2}$-space, resulting in a more efficient utilization of this space. This indeed is the difference in the sum rate achievable using independent unstructured codes and PZ-technique. We also note the decoder does not attempt to disambiguate the pair $\left(U_{1}^{n}, U_{2}^{n}\right)$ and restricts to decoding $U_{1}^{n} \oplus_{2} U_{2}^{n}$. This is motivated by the Markov chain $\left(U_{1}, U_{2}\right)-U_{1} \oplus_{2} U_{2}-Y$ induced by the test channel and the use of structured codebooks that contain the sum.

It is instructive to investigate the efficacy of this technique if users 1 and 2 employ distinct linear codes $\lambda_{I 1}, \lambda_{I 2}$ of rate $1-h_{b}(\tau)$ instead of a common linear code $\lambda_{I}$. In this case, each message of user 1 can result in $2^{2-2 h_{b}(\tau)}$ received vectors which restricts user 1 's rate to $2 h_{b}(q)-1$ and provides no improvement over the unstructured coding technique. We conclude that if the bins of the MAC channel code are nontrivial, as in this case due to the presence of a state, then it maybe beneficial to endow the bins with an algebraic structure that restricts the range of a bivariate function, and enable the decoder decode this function of chosen codewords.

\section{B. Stage I : An achievable rate region for MAC-DSTx using nested coset codes}

In this section, we present the first stage of our coding scheme that uses joint typical encoding and decoding and nested coset codes over an arbitrary MAC-DSTx. The technique proposed by Philosof and Zamir is specific to the binary doubly dirty MAC - Hamming cost constraint that induces additive test channels between the auxiliary and state random variables, and additive and symmetric nature of the channel. Moreover, linear codes only achieve the symmetric capacity, and therefore if the output were obtained by passing $\left(X_{1}^{n} \oplus_{2} S_{1}^{n}, X_{2}^{n} \oplus_{2} S_{2}^{n}\right)$ through an asymmetric MAC, linear codes though applicable, might not be optimal.

We begin with a characterization of test channels followed by achievability.

Definition 10: Let $\mathbb{D}_{f}(\boldsymbol{\tau}) \subseteq \mathbb{D}(\boldsymbol{\tau})$ be the collection of distributions $p_{\boldsymbol{V} \boldsymbol{S} \boldsymbol{X} Y}$ on $\mathcal{V}^{2} \times \mathcal{S} \times \mathcal{X} \times \mathcal{Y}$ where $\mathcal{V}$ is a finite field. For $p_{\boldsymbol{V} \boldsymbol{X} \boldsymbol{S} Y} \in \mathbb{D}_{f}(\boldsymbol{\tau})$, let $\beta_{f}\left(p_{\boldsymbol{V} \boldsymbol{X} \boldsymbol{S} Y}\right)$ be defined as the set

$$
\left\{\left(R_{1}, R_{2}\right) \in[0, \infty)^{2}: R_{1}+R_{2} \leq \min \left\{H\left(V_{1} \mid S_{1}\right), H\left(V_{2} \mid S_{2}\right)\right\}-H\left(V_{1} \oplus V_{2} \mid Y\right)\right\} .
$$

Let

$$
\beta_{f}(\boldsymbol{\tau}):=\operatorname{cocl}\left(\bigcup_{p_{\boldsymbol{V} \boldsymbol{X} S Y} \in \mathbb{D}_{f}(\boldsymbol{\tau})} \beta_{f}\left(p_{\boldsymbol{V} \boldsymbol{X} \boldsymbol{S} Y}\right)\right)
$$

Theorem 4: $\beta_{f}(\boldsymbol{\tau}) \subseteq \mathbb{C}(\boldsymbol{\tau})$.

Before we provide a proof, we state the coding technique and indicate achievability of promised rates. As stated in section VI-A, the key aspect is to employ cosets of a common linear code as bins for quantizing 
the state. We employ three nested coset codes -one each for the two encoders and the decoder- that share a common inner (sparser) code. We begin by describing the encoding rule. The nested coset code provided to encoder $j$ is described through a pair of generator matrices $g_{I} \in \mathcal{V}^{k \times n}$ and $g_{O j / I} \in \mathcal{V}^{l_{j} \times n}$ where (i) $g_{I}$ and $g_{O j}^{T}:=\left[\begin{array}{ll}g_{I}^{T} & g_{O j / I}^{T}\end{array}\right]$ are generator matrices of inner (sparser) and complete (denser) codes respectively, (ii)

$$
\begin{aligned}
\frac{k}{n} & >1-\frac{\min \left\{H\left(V_{1} \mid S_{1}\right), H\left(V_{2} \mid S_{2}\right)\right\}}{\log \pi} \\
\frac{k+l_{1}+l_{2}}{n} & <1-\frac{H\left(V_{1} \oplus V_{2} \mid Y\right)}{\log \pi} .
\end{aligned}
$$

with $\pi:=|\mathcal{V}|$ and (iii) bias vector $b_{j}^{n}$. Let $\lambda_{I}$ and $\lambda_{O j}$ denote linear codes corresponding to generator matrices $g_{I}$ and $g_{O j}$ respectively. User $j$ 's message $M_{j}^{l_{j}} \in \mathcal{V}^{l_{j}}$ indexes the $\operatorname{coset}\left(a^{k} g_{I} \oplus M_{j}^{l_{j}} g_{O j / I} \oplus b_{j}^{n}\right.$ : $a^{k} \in \mathcal{V}^{k}$ ). Encoder $j$ observes state $S_{j}^{n}$ and looks for a codeword in the coset indexed by the message that is jointly typical with the state sequence $S_{j}^{n}$ according to $p_{S_{j} V_{j}}$. If it finds one such codeword, say $V_{j}^{n}$, a vector $X_{j}^{n}$ is generated according $\prod_{t=1}^{n} p_{X_{j} \mid S_{j} V_{j}}\left(\cdot \mid S_{j t} V_{j t}\right)$ and $X_{j}^{n}$ is fed as input to the channel. Otherwise, it declares an error.

Now to the decoding rule. Let $\lambda_{O}$ denote the complete code provided to the decoder, i.e., the coset code whose (i) generator matrix is $g_{O}^{T}:=\left[\begin{array}{ll}g_{I}^{T} & g_{O / I}^{T}\end{array}\right]$, where $g_{O / I}^{T}:=\left[\begin{array}{ll}g_{O 1 / I}^{T} & g_{O 2 / I}^{T}\end{array}\right]$ and (ii) bias vector $b_{1}^{n} \oplus b_{2}^{n}$. Having received $Y^{n}$, it lists all codewords in $\lambda_{O}$ that are jointly typical with $Y^{n}$ with respect to $p_{V_{1} \oplus V_{2}, Y}$. If all such codewords belong to a unique coset (of $\lambda_{I}$ in $\left.\lambda_{O}\right)$ say $\left(a^{k} g_{I} \oplus m_{1}^{l_{1}} g_{O 1 / I} \oplus\right.$ $\left.m_{2}^{l_{2}} g_{O 2 / I} \oplus b_{1}^{n} \oplus b_{2}^{n}: a^{k} \in \mathcal{V}^{k}\right)$, it declares $\left(m_{1}^{l_{1}}, m_{2}^{l_{2}}\right)$ as the pair of decoded messages. Otherwise, it declares an error.

We pick entries of each of the constituent generator matrices $g_{I}, g_{O 1 / I}, g_{O 2 / I}$ independently and uniformly from $\mathcal{V}$. Lower bound (13) enable us to drive down the probability of encoder not finding a jointly typical codeword in the indexed coset. This bound can be interpreted easily. If we picked codewords according to $\prod_{t=1}^{n} p_{V}$, then we need the bin to be of rate roughly $H\left(V_{1}\right)-H\left(V_{1} \mid S_{1}\right)$. Since we average uniformly over the ensemble of coset codes, each codeword of a linear code is uniformly distributed over $\mathcal{V}^{n}$. Hence the bin must of rate at least $\log \pi-H\left(V_{1} \mid S_{1}\right)$. The decoder makes an error with arbitrarily small probability if (14) is satisfied. This bound can also be interpreted intuitively. If the codewords were picked according to $p_{V_{1} \oplus V_{2}}$, the upper bound would have been $H\left(V_{1} \oplus V_{2}\right)-H\left(V_{1} \oplus V_{2} \mid Y\right)$. In this case, the codewords in the sum of nested linear codes are also uniformly distributed over $\mathcal{V}^{n}$, and this explains the bound in (14). From (13), (14) it can be verified that $R_{1}+R_{2}=\frac{l_{1}+l_{2}}{n} \leq \min \left\{H\left(V_{1} \mid S_{1}\right), H\left(V_{2} \mid S_{2}\right)-H\left(V_{1} \oplus V_{2} \mid Y\right)\right\}$ is achievable.

We emphasize that joint typical encoding and decoding enables us to decode the sum over an arbitrary 
MAC-DSTx. The informed reader will recognize the need to prove statistical independence of a codeword in a competing sum coset and the pair of cosets indexed by the messages. The dependence built across the codewords and cosets as a consequence of the algebraic structure exemplifies the interplay of algebra and probability. The following proof details these elements.

Proof: Let pmf $p_{\boldsymbol{V} \boldsymbol{X} \boldsymbol{S} Y} \in \mathbb{D}_{f}(\boldsymbol{\tau})$, rate pair $\mathbf{R} \in \beta_{f}\left(p_{\boldsymbol{V} \boldsymbol{X} \boldsymbol{S} Y}\right)$ and $\eta>0$. We prove existence of a MAC-DSTx code $(n, \mathscr{M}, \mathbf{e}, d)$ whose rate $\frac{\log \mathscr{M}_{j}}{n} \geq R_{j}-\eta$, average error probability $\bar{\xi}(\mathbf{e}, d) \leq \eta$, and average cost $\tau\left(e_{j}\right) \leq \tau_{j}+\eta$ for $j=1,2$.

We begin with a description of the structure of the MAC-DSTx code whose existence we seek to prove. Let $\pi:=|\mathcal{V}|$ and we assume $H\left(V_{1} \mid S_{1}\right) \geq H\left(V_{2} \mid S_{2}\right)$ without loss of generality. Consider a pair of nested coset codes $\left(n, k_{j}, l_{j}, g_{I_{j}}, g_{O_{j} / I_{j}}, b_{j}^{n}\right): j=1,2$ built over $\mathcal{V}$, denoted $\lambda_{O_{j}} / \lambda_{I_{j}}: j=1,2$ with parameters

$$
\begin{aligned}
& k_{1}:=\left\lceil n\left(1-\frac{H\left(V_{1} \mid S_{1}\right)}{\log \pi}+\frac{\eta_{1}(\eta)}{\log \pi}\right)\right\rceil, \\
& k_{2}=k_{1}+k_{+}, \text {where } k_{+}:=\left\lceil n\left(1-\frac{H\left(V_{2} \mid S_{2}\right)}{\log \pi}+\frac{\eta_{1}(\eta)}{\log \pi}\right)\right\rceil-k_{1}, \\
& l_{1}:=\left\lfloor n\left(\frac{R_{1}}{\log \pi}-\frac{\eta_{2}(\eta)}{\log \pi}\right)\right\rfloor \\
& l_{2}:=\left\lfloor n\left(1+\frac{R_{2}}{\log \pi}-\frac{H\left(V_{2} \mid S_{2}\right)}{\log \pi}-\frac{\eta_{3}(\eta)}{\log \pi}\right)\right\rfloor-k_{2}, \text { and, }
\end{aligned}
$$

the first $k_{1}$ rows of $g_{I_{1}}$ and $g_{I_{2}}$ are identical i.e., $g_{I_{1}, t}=g_{I_{2}, t}$ for $t \in\left[k_{1}\right]$.

A few remarks on the structure of $\lambda_{O_{j}} / \lambda_{I_{j}}: j=1,2$ and the relationship between their parameters are in order. For $n \geq N_{1}(\eta):=\max \left\{\frac{\log \pi}{\eta_{1}(\eta)}, \frac{\log \pi}{\eta_{2}(\eta)}, \frac{\log \pi}{\eta_{3}(\eta)}\right\}$, we have

$$
\begin{aligned}
& \frac{n}{\log \pi}\left(\log \pi-H\left(V_{j} \mid S_{j}\right)+\eta_{1}(\eta)\right) \leq \quad k_{j} \quad \leq \frac{n}{\log \pi}\left(\log \pi-H\left(V_{j} \mid S_{j}\right)+2 \eta_{1}(\eta)\right) \\
& \frac{n}{\log \pi}\left(R_{1}-2 \eta_{2}(\eta)\right) \leq \quad l_{1} \quad \leq \frac{n}{\log \pi}\left(R_{1}-\eta_{2}(\eta)\right) \\
& \frac{n}{\log \pi}\left(R_{2}+\log \pi-H\left(V_{2} \mid S_{2}\right)-2 \eta_{3}(\eta)\right) \leq k_{2}+l_{2} \leq \frac{n}{\log \pi}\left(R_{2}+\log \pi-H\left(V_{2} \mid S_{2}\right)-\eta_{3}(\eta)\right)(22)
\end{aligned}
$$

Combining the lower bound in 22) and the upper bound for $k_{2}$ in 20, we have

$$
\frac{l_{2} \log \pi}{n} \geq R_{2}-2 \eta_{3}(\eta)-2 \eta_{1}(\eta)
$$

and similarly, combining the upper bound for $k_{2}+l_{2}$ in 22) and the upper bound for $l_{1}$ in (21), we have

$$
\begin{aligned}
k_{2}+l_{1}+l_{2} & \leq \frac{n}{\log \pi}\left(R_{1}+R_{2}+\log \pi-H\left(V_{2} \mid S_{2}\right)-\eta_{3}(\eta)-\eta_{2}(\eta)\right) \\
& \leq \frac{n}{\log \pi}\left(\log \pi-H\left(V_{1} \oplus V_{2} \mid Y\right)-\eta_{3}(\eta)-\eta_{2}(\eta)\right),
\end{aligned}
$$


where 24) follows from $\mathbf{R} \in \beta_{f}\left(p_{\boldsymbol{V} X \boldsymbol{S} Y}\right)$.

We now specify encoding and decoding rules that map this pair $\lambda_{O_{j}} / \lambda_{I_{j}}: j=1,2$ of nested coset codes into a MAC-DSTx code. User $j$ is provided with the nested coset code $\lambda_{O_{j}} / \lambda_{I_{j}}$. User $j$ 's message is used to index one among $\pi^{l_{j}}$ cosets of $\lambda_{O_{j}} / \lambda_{I_{j}}$. We assume that the set of messages $\mathcal{M}_{j}:=\mathcal{V}^{l_{j}}$, and $M_{j}^{l_{j}} \in \mathcal{V}^{l_{j}}$ to be the uniformly distributed random variable representing user $j$ 's message. We let $v_{j}^{n}\left(a_{j}^{k_{j}}, m_{j}^{l_{j}}\right):=a_{j}^{k_{j}} g_{I_{j}} \oplus m_{j}^{l_{j}} g_{O_{j} / I_{j}} \oplus b_{j}^{n}$ denote a generic codeword in $\lambda_{O_{j}} / \lambda_{I_{j}}$ and $c_{j}\left(m_{j}^{l_{j}}\right)$ : $=\left(v_{j}^{n}\left(a_{j}^{k_{j}}, m_{j}^{l_{j}}\right): a_{j}^{k_{j}} \in \mathcal{V}^{k_{j}}\right)$ denote the coset corresponding to message $m_{j}^{l_{j}}$. Encoder $j$ observes the state sequence $S_{j}^{n}$ and populates the list $L_{j}\left(M_{j}^{l_{j}}, S_{j}^{n}\right)=\left\{v_{j}\left(a_{j}^{k_{j}}, M_{j}^{l_{j}}\right):\left(S_{j}^{n}, v_{j}\left(a_{j}^{k_{j}}, M_{j}^{l_{j}}\right)\right) \in T_{\eta_{4}(\eta)}\left(S_{j}, V_{j}\right)\right\}$ of codewords in the coset corresponding to the message that are jointly typical with the state sequence. If $L_{j}\left(M_{j}^{l_{j}}, S_{j}^{n}\right)$ is empty, it picks a codeword uniformly at random from coset $c_{j}\left(M_{j}^{l_{j}}\right)$. Otherwise, it picks a codeword uniformly at random from $L_{j}\left(M_{j}^{l_{j}}, S_{j}^{n}\right)$. Let $V_{j}\left(A_{j}^{k_{j}}, M_{j}^{l_{j}}\right)$ denote the picked codeword in either case. The encoder computes $X_{j}^{n}\left(M_{j}^{l_{j}}, S_{j}^{n}\right):=f_{j}^{n}\left(V_{j}^{n}\left(A_{j}^{k_{j}}, M_{j}^{l_{j}}\right), S_{j}^{n}\right)$, where $f_{j}: \mathcal{V}_{j} \times \mathcal{S}_{j} \rightarrow \mathcal{X}_{j}$ is any map that satisfies $p_{X_{j} \mid V_{j} S_{j}}\left(f_{j}\left(v_{j}, s_{j}\right) \mid v_{j}, s_{j}\right)=1$ for all pairs $\left(v_{j}, s_{j}\right) \in \mathcal{V}_{j} \times \mathcal{S}_{j} . X_{j}^{n}\left(M_{j}^{l_{j}}, S_{j}^{n}\right)$ is fed as input to the channel.

We now specify the decoding rule. The decoder is provided with nested coset code $\left(n, k, l, g_{I}, g_{O / I}, b^{n}\right)$ denoted $\lambda_{O} / \lambda_{I}$ where $k=k_{2}, l=l_{1}+l_{2}, g_{I}=g_{I_{2}}, g_{O / I}^{T}:=\left[\begin{array}{ll}g_{O_{1} / I_{1}}^{T} & g_{O_{2} / I_{2}}^{T}\end{array}\right]$ and $b^{n}:=b_{1}^{n} \oplus b_{2}^{n}$. With a slight abuse of notation, we let $m^{l}:=\left(m_{1}^{l_{1}}, m_{2}^{l_{2}}\right) \in \mathcal{V}^{l}:=\mathcal{V}^{l_{1}} \times \mathcal{V}^{l_{2}}$ represent a pair of messages and analogously random variable $M^{l}:=\left(M_{1}^{l_{1}}, M_{2}^{l_{2}}\right)$ denote the pair of user messages. For $a^{k} \in \mathcal{V}^{k}$ and $m^{l} \in \mathcal{V}^{l}$, let $v^{n}\left(a^{k}, m^{l}\right):=a^{k} g_{I} \oplus m^{l} g_{O / I} \oplus b^{n}$ and $c\left(m^{l}\right):=\left(v^{n}\left(a^{k}, m^{l}\right): a^{k} \in \mathcal{V}^{k}\right)$ denote a generic codeword in $\lambda_{O} / \lambda_{I}$ and the coset corresponding to the message pair $m^{l}$ respectively. The decoder observes the received vector $Y^{n}$ and populates $D\left(Y^{n}\right):=\left\{m^{l} \in \mathcal{V}^{l}: \exists v^{n}\left(a^{k}, m^{l}\right)\right.$ such that $\left.\left(v^{n}\left(a^{k}, m^{l}\right), Y^{n}\right) \in T_{\eta_{5}(\eta)}\left(V_{1} \oplus V_{2}, Y\right)\right\}$. If $D\left(Y^{n}\right)$ is a singleton, the decoder declares the content of $D\left(Y^{n}\right)$ as the decoded message pair. Otherwise, it declares an error.

The above encoding and decoding rules map every pair $\lambda_{O_{j}} / \lambda_{I_{j}}: j=1,2$ of nested coset codes that satisfy $15-19$ into a corresponding MAC-DSTx code $\left(n, \mathscr{M}^{(n)}, \mathbf{e}^{(n)}, d^{(n)}\right)$ of rate $\frac{\log \mathscr{M}_{j}^{(n)}}{n} \geq$ $R_{j}-2 \eta_{1}(\eta)-2 \eta_{2}(\eta)$, thus characterizing an ensemble, one for each $n$, of MAC-DSTx codes. We average the error probability over this ensemble of MAC-DSTx codes by letting the bias vectors $B_{j}^{n}: j=1,2$ and generator matrices $G_{I_{2}}, G_{O_{j} / I_{j}}: j=1,2$ mutually independent and uniformly distributed over their respective range spaces. Let $\Lambda_{O_{j}} / \Lambda_{I_{j}}: j=1,2$ and $\Lambda_{O} / \Lambda_{I}$ denote the random nested coset codes $\left(n, k_{j}, l_{j}, G_{I_{j}}, G_{O_{j} / I_{j}}, B_{j}^{n}\right): j=1,2$ and $\left(n, k, l, G_{I}, G_{O / I}, B^{n}\right)$ respectively. For $a_{j}^{k_{j}} \in \mathcal{V}^{k_{j}}$, $m_{j}^{l_{j}} \in \mathcal{V}^{l_{j}}, a^{k} \in \mathcal{V}^{k}, m^{l} \in \mathcal{V}^{l}$, let $V_{j}^{n}\left(a_{j}^{k_{j}}, m_{j}^{l_{j}}\right):=a_{j}^{k_{j}} G_{I_{j}} \oplus m_{j}^{l_{j}} G_{O_{j} / I_{j}} \oplus B_{j}^{n}: j=1,2, V^{n}\left(a^{k}, m^{l}\right):$ $=a^{k} G_{I} \oplus m^{l} G_{O / I} \oplus B^{n}$ denote corresponding random codewords in $\Lambda_{O_{j}} / \Lambda_{I_{j}}: j=1,2$ and $\Lambda_{O} / \Lambda_{I}$ 
respectively. Let $C_{j}\left(m_{j}^{l_{j}}\right):=\left(V_{j}^{n}\left(a_{j}^{k_{j}}, m_{j}^{l_{j}}\right): a_{j}^{k_{j}} \in \mathcal{V}^{k_{j}}\right)$ and $C\left(m^{l}\right):=\left(V^{n}\left(a^{k}, m^{l}\right): a^{k} \in \mathcal{V}^{k}\right)$ denote random cosets in $\Lambda_{O_{j}} / \Lambda_{I_{j}}: j=1,2$ and $\Lambda_{O} / \Lambda_{I}$ corresponding to message $m_{j}^{l_{j}}: j=1,2$ and $m^{l}$ respectively.

Our next goal is to derive an upper bound on the probability of error. Towards this end, we begin with a characterization of related events. Let

$$
\begin{aligned}
\epsilon_{1 j} & :=\left\{S_{j}^{n} \notin T_{\frac{\eta_{4}(\eta)}{2}}\left(S_{j}\right)\right\}, \quad \epsilon_{1}:=\left\{\boldsymbol{S}^{n} \notin T_{\frac{\eta_{4}(\eta)}{2}}(\boldsymbol{S})\right\} \\
\epsilon_{2 j} & :=\left\{\phi_{j}\left(S_{j}^{n}, M_{j}^{l_{j}}\right)=0\right\}, \text { where } \phi_{j}\left(s_{j}^{n}, m_{j}^{l_{j}}\right):=\sum_{a_{j}^{k_{j}} \in \mathcal{V}^{k_{j}}} 1_{\left\{\left(V_{j}^{n}\left(a_{j}^{k_{j}}, m_{j}^{l_{j}}\right), s_{j}^{n}\right) \in T_{\eta_{4}(\eta)}\left(V_{j}, S_{j}\right)\right\}} \\
\epsilon_{4} & :=\bigcup_{a^{k} \in \mathcal{V}^{k}}\left\{\left(V^{n}\left(a^{k}, M^{l}\right), Y^{n}\right) \in T_{\eta_{5}(\eta)}\left(V_{1} \oplus V_{2}, Y\right)\right\} \\
\epsilon_{5} & :=\bigcup_{\hat{m}^{l} \neq M^{l} a^{k} \in \mathcal{V}^{k}}\left\{\left(V^{n}\left(a^{k}, \hat{m}^{l}\right), Y^{n}\right) \in T_{\eta_{5}(\eta)}\left(p_{V_{1} \oplus V_{2}, Y}\right)\right\} .
\end{aligned}
$$

Note that $\epsilon_{1} \cup \epsilon_{21} \cup \epsilon_{22} \cup \epsilon_{4}^{c} \cup \epsilon_{5}$ contains the error event and hence $P\left(\epsilon_{1}\right)+P\left(\epsilon_{11}^{c} \cap \epsilon_{21}\right)+P\left(\epsilon_{12}^{c} \cap\right.$ $\left.\epsilon_{22}\right)+P\left(\left(\epsilon_{1} \cup \epsilon_{21} \cup \epsilon_{22}\right)^{c} \cap \epsilon_{4}^{c}\right)+P\left(\epsilon_{5}\right)$ is an upper bound on the probability of error. In the sequel, we provide an upper bound on each of the above terms.

Lemma 2 guarantees the existence of $N_{2}(\eta) \in \mathbb{N}$ such that $P\left(\epsilon_{1}\right) \leq \frac{\eta}{8}$ for all $n \geq N_{1}(\eta)$. Lemma 7 (3) in appendix $\mathrm{A}$ implies the existence of $N_{3}(\eta) \in \mathbb{N}$ such that for all $n \geq N_{3}(\eta)$

$$
P\left(\epsilon_{1 j}^{c} \cap \epsilon_{2 j}\right) \leq \exp \left\{-n \log \pi\left(\frac{k_{j}}{n}-\left(1-\frac{H\left(V_{j} \mid S_{j}\right)}{\log \pi}+\frac{3 \eta_{4}(\eta)}{2 \log \pi}\right)\right)\right\} .
$$

Substituting the lower bound in 20 for $\frac{k_{j}}{n}$, we obtain

$$
P\left(\epsilon_{1 j}^{c} \cap \epsilon_{2 j}\right) \leq \exp \left\{-n\left(\eta_{1}(\eta)-\frac{3 \eta_{4}(\eta)}{2}\right)\right\} .
$$

for all $n \geq \max \left\{N_{1}(\eta), N_{3}(\eta)\right\}$. We now derive an upper bound on $P\left(\left(\epsilon_{1} \cup \epsilon_{21} \cup \epsilon_{22}\right)^{c} \cap \epsilon_{4}^{c}\right)$. The encoding rule ensures $\left(\epsilon_{1} \cup \epsilon_{21} \cup \epsilon_{22}\right)^{c} \subseteq\left(\epsilon_{1} \cup \epsilon_{2}\right)^{c}$, where

$$
\epsilon_{2}=\bigcup_{j=1}^{2}\left\{\left(S_{j}^{n}, V_{j}^{n}\left(A_{j}^{k_{j}}, M_{j}^{l_{j}}\right)\right) \notin T_{\eta_{4}(\eta)}\left(S_{j}, V_{j}\right)\right\},
$$

and $V_{j}^{n}\left(A_{j}^{k_{j}}, M_{j}^{l_{j}}\right)$ denotes codeword in $L_{j}\left(M_{j}^{l_{j}}, S_{j}^{n}\right)$ chosen by encoder $j$. Our first step is to provide an upper bound on $P\left(\left(\epsilon_{1} \cup \epsilon_{2}\right)^{c} \cap \epsilon_{3}\right)$ for sufficiently large $n$, where

$$
\epsilon_{3}=\left\{\left(S_{j}^{n}, V_{j}^{n}\left(A_{j}^{k_{j}}, M_{j}^{l_{j}}\right): j=1,2\right) \notin T_{\frac{\eta_{5}(\eta)}{2}}\left(S_{1}, V_{1}, S_{2}, V_{2}\right)\right\} .
$$

In the second step, we employ the result of conditional frequency typicality to provide an upper bound on $P\left(\left(\epsilon_{1} \cup \epsilon_{2} \cup \epsilon_{3}\right)^{c} \cap \epsilon_{4}^{c}\right)$. 
As an astute reader might have guessed, the proof of first step will employ the Markov chain $V_{1}-$ $S_{1}-S_{2}-V_{2}$. The proof is non-trivial because of statistical dependence of the codebooks. We begin with the definition

$$
\Theta\left(s^{n}\right):=\left\{\boldsymbol{v}^{n} \in \mathcal{V}^{n}:\left(s_{j}^{n}, v_{j}^{n}\right) \in T_{\eta_{4}(\eta)}\left(S_{j}, V_{j}\right): j=1,2,\left(\boldsymbol{s}^{n}, \boldsymbol{v}^{n}\right) \notin T_{\frac{\eta_{5}(\eta)}{2}}(\boldsymbol{S}, \boldsymbol{V})\right\}
$$

for any $s^{n} \in \mathcal{S}^{n}$. Observe that,

$$
\begin{aligned}
& P\left(\left(\epsilon_{1} \cup \epsilon_{2}\right)^{c} \cap \epsilon_{3}\right)=\sum_{\boldsymbol{s}^{n} \in T_{\frac{\eta_{4}(\eta)}{2}}(\boldsymbol{S})} \sum_{\boldsymbol{v}^{n} \in \Theta\left(\boldsymbol{s}^{n}\right)} P\left(\boldsymbol{S}^{n}=s^{n}, V_{j}^{n}\left(A_{j}^{k_{j}}, M_{j}^{l_{j}}\right)=v_{j}^{n}: j=1,2\right) \\
& =\sum_{\boldsymbol{s}^{n} \in T_{\frac{\eta_{4}(\eta)}{2}}(\boldsymbol{S})} \sum_{\boldsymbol{v}^{n} \in \Theta\left(\boldsymbol{s}^{n}\right)} P\left(\bigcup_{a_{1}^{k_{1}} \in \mathcal{V}_{1}^{k_{1}}} \bigcup_{a_{2}^{k_{2}} \in \mathcal{V}_{2}^{k_{2}}}\left\{\boldsymbol{S}^{n}=\boldsymbol{s}^{n}, \begin{array}{l}
V_{j}^{n}\left(A_{j}^{k_{j}}, M_{j}^{l_{j}}\right)=v_{j}^{n}: j=1,2, \\
V_{j}^{n}\left(a_{j}^{k_{j}}, M_{j}^{l_{j}^{j}}\right)=v_{j}^{n}: j=1,2
\end{array}\right\}\right) \\
& \leq \sum_{\boldsymbol{s}^{n} \in T_{\frac{\eta_{4}(\eta)}{2}}} \sum_{\boldsymbol{S})} \sum_{\boldsymbol{v}^{n} \in \Theta\left(\boldsymbol{s}^{n}\right)} \sum_{a_{1}^{k_{1}} \in \mathcal{V}_{1}^{k_{1}}} P\left(\left\{\boldsymbol{S}^{k_{2}} \in \mathcal{V}_{2}^{k_{2}}=\boldsymbol{s}^{n}, \begin{array}{l}
V_{1}^{n}\left(a_{1}^{k_{1}}, M_{1}^{k_{1}}\right)=v_{1}^{n}, \\
V_{2}^{n}\left(a_{2}^{k_{2}}, M_{2}^{l_{2}}\right)=v_{2}^{n}
\end{array}\right\}\right) \\
& =\sum_{\boldsymbol{s}^{n} \in T_{\frac{\eta_{4}(\eta)}{2}}} \sum_{\boldsymbol{S})} \sum_{\boldsymbol{v}^{n} \in \Theta\left(\boldsymbol{s}^{n}\right)} \sum_{a_{1}^{k_{1}} \in \mathcal{V}_{1}^{k_{1}}} \sum_{a_{2}^{k_{2}} \in \mathcal{V}_{2}^{k_{2}}} P\left(\boldsymbol{S}^{n}=\boldsymbol{s}^{n}\right) P\left(\begin{array}{c}
V_{1}^{n}\left(a_{1}^{k_{1}}, M_{1}^{l_{1}}\right)=v_{1}^{n}, \\
V_{2}^{n}\left(a_{2}^{k_{2}}, M_{2}^{l_{2}}\right)=v_{2}^{n}
\end{array}\right) \\
& =\sum_{\boldsymbol{s}^{n} \in T_{\frac{\eta_{4}(\eta)}{2}}} \sum_{\boldsymbol{S}) \boldsymbol{v}^{n} \in \Theta\left(\boldsymbol{s}^{n}\right)} P\left(\boldsymbol{S}^{n}=\boldsymbol{s}^{n}\right) \frac{1}{\pi^{n-k_{1}}} \frac{1}{\pi^{n-k_{2}}}
\end{aligned}
$$

where $V_{j}^{n}\left(A_{j}^{k_{j}}, M_{j}^{l_{j}}\right)$ is defined as the random codeword chosen by the encoder, 26 follows from independence of random variables $\left(M^{l}, G_{I}, G_{O / I}, B_{1}^{n}, B_{2}^{n}\right)$ that characterize $V_{j}^{n}\left(a_{j}^{k_{j}}, M_{j}^{l_{j}}\right)$ and $\boldsymbol{S}^{n}$. We now employ the upper bound on $k_{j}$ in 20 to substitute for $\frac{1}{\pi^{n-k_{j}}}$. For $n \geq N_{1}(\eta)$, we have $k_{j} \leq$ $n-\frac{H\left(V_{j} \mid S_{j}\right)}{\log \pi}+\frac{2 \eta_{1}(\eta)}{\log \pi}$ and hence

$$
\frac{1}{\pi^{n-k_{j}}} \leq \exp \left\{-n\left(H\left(V_{j} \mid S_{j}\right)-2 \eta_{1}(\eta)\right)\right\} .
$$

Furthermore, by Lemma 1 , for every $\boldsymbol{s}^{n} \in T_{\frac{\eta_{4}(\eta)}{2}}(\boldsymbol{S})$ and $\boldsymbol{v}^{n} \in \Theta\left(\boldsymbol{s}^{n}\right)$,

$$
\exp \left\{-n\left(H\left(V_{j} \mid S_{j}\right)-2 \eta_{4}(\eta)\right)\right\} \leq p_{V_{j}^{n} \mid S_{j}^{n}}\left(v_{j}^{n} \mid s_{j}^{n}\right)=p_{V_{j}^{n} \mid \boldsymbol{S}^{n}}\left(v_{j}^{n} \mid s^{n}\right)=p_{V_{j}^{n} \mid \boldsymbol{S}^{n} V_{j}^{n}}\left(v_{j}^{n} \mid s^{n}, v_{\dot{j}}^{n}\right),
$$

where the last equalities is a consequence of Markov chain $V_{1}-S_{1}-S_{2}-V_{2}$. Substituting the upper bounds in 28 and 29 for $\frac{1}{\pi^{n-k_{j}}}$ in 27 , we obtain

$$
\begin{aligned}
P\left(\left(\epsilon_{1} \cup \epsilon_{2}\right)^{c} \cap \epsilon_{3}\right) & \leq \exp \left\{n\left(4 \eta_{1}(\eta)+4 \eta_{4}(\eta)\right)\right\} \cdot \sum_{\boldsymbol{s}^{n} \in T_{\frac{\eta_{4}(\eta)}{2}}(\boldsymbol{S})} \sum_{\boldsymbol{v}^{n} \in \Theta\left(\boldsymbol{s}^{n}\right)} p_{\boldsymbol{S}^{n}} \boldsymbol{V}^{n}\left(\boldsymbol{s}^{n}, \boldsymbol{v}^{n}\right) \\
& \leq \exp \left\{n\left(4 \eta_{1}(\eta)+4 \eta_{4}(\eta)\right)\right\} \cdot \sum_{\left(\boldsymbol{s}^{n}, \boldsymbol{v}^{n}\right) \notin T_{\eta_{5}(\eta)}(\boldsymbol{S}, \boldsymbol{V})} p_{\boldsymbol{S}^{n} \boldsymbol{V}^{n}\left(\boldsymbol{s}^{n}, \boldsymbol{v}^{n}\right)}
\end{aligned}
$$


for all $n \geq N_{1}(\eta)$. We now employ the exponential upper bound provided in Lemma 2. In particular, Lemma 2 guarantees the existence of $N_{4}(\eta) \in \mathbb{N}$ such that for every $n \geq N_{4}(\eta)$,

$$
\sum_{\substack{\left(\boldsymbol{s}^{n}, \boldsymbol{v}^{n}\right) \in \\ T_{\eta_{5}(\eta)}(\boldsymbol{S}, \boldsymbol{V})}} p_{\boldsymbol{S}^{n} \boldsymbol{V}^{n}}\left(\boldsymbol{s}^{n}, \boldsymbol{v}^{n}\right) \leq \exp \left\{-n \lambda \eta_{5}^{2}(\eta)\right\}, \text { where } \lambda:=\frac{\min _{(\boldsymbol{s}, \boldsymbol{v}) \in \mathcal{S} \times \mathcal{V}}\left\{p_{\boldsymbol{S} \boldsymbol{V}}^{2}(\boldsymbol{s}, \boldsymbol{v}): p_{\boldsymbol{S} \boldsymbol{V}}(\boldsymbol{s}, \boldsymbol{v})>0\right\}}{(\log |\mathcal{S} \| \mathcal{V}|)^{2}} .
$$

Substituting (31) in 30, we conclude

$$
P\left(\left(\epsilon_{1} \cup \epsilon_{2}\right)^{c} \cap \epsilon_{3}\right) \leq \exp \left\{-n\left(\lambda \eta_{5}^{2}(\eta)-4 \eta_{1}(\eta)-4 \eta_{4}(\eta)\right)\right\}
$$

for every $n \geq \max \left\{N_{1}(\eta), N_{4}(\eta)\right\}$. This gets us to the second step. We begin with two observations. Firstly, note that $V\left(a_{1}^{k_{1}} 0^{k_{+}} \oplus a_{2}^{k_{2}}, m_{1}^{l_{1}} m_{2}^{l_{2}}\right)=V_{1}\left(a_{1}^{k_{1}}, m_{1}^{l_{1}}\right) \oplus V_{2}\left(a_{2}^{k_{2}}, m_{2}^{l_{2}}\right)$. This follows from the definition of the codewords involved. Secondly,

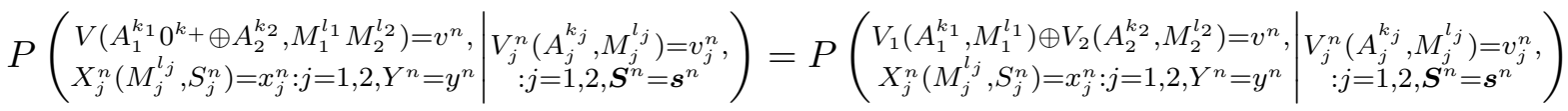

$$
\begin{aligned}
& =\prod_{t=1}^{n}\left[p_{V_{1} \oplus V_{2} \mid V_{1} V_{2}}\left(v_{t} \mid v_{1 t}, v_{2 t}\right)\left(\prod_{j=1}^{2} p_{X_{j} \mid V_{j} S_{j}}\left(x_{j t} \mid v_{j t}, s_{j t}\right)\right) W_{Y \mid \boldsymbol{X} \boldsymbol{S}}\left(y_{t} \mid \boldsymbol{x}_{t}, \boldsymbol{s}_{t}\right)\right] \\
& =\prod_{t=1}^{n} P\left(V_{1} \oplus V_{2}=v_{t}, \boldsymbol{X}=\boldsymbol{x}_{t}, Y_{t}=y_{t} \mid \boldsymbol{S}_{t}=\boldsymbol{s}_{t}, \boldsymbol{V}_{t}=\boldsymbol{v}_{t}\right),
\end{aligned}
$$

where we have employed 1) encoding rule and Markov chains $\boldsymbol{U}-(\boldsymbol{X}, \boldsymbol{S})-Y$ in arriving at 33 and 2) the identity $p_{X_{j} \mid S \boldsymbol{U} X_{\dot{f}}}=p_{X_{j} \mid S \boldsymbol{U}}=p_{X_{j} \mid S_{j} U_{j}}$ for any distinct elements $j, \dot{f} \in\{1,2\}$ in arriving at (34).

Since

$$
\begin{aligned}
& P\left(\left(\epsilon_{1} \cup \epsilon_{2} \cup \epsilon_{3}\right)^{c} \cap \epsilon_{4}^{c}\right) \leq P\left(\left(\epsilon_{1} \cup \epsilon_{2} \cup \epsilon_{3}\right)^{c} \cap\left\{\left(V\left(\begin{array}{c}
A_{1}^{k_{1}} 0^{k_{+}} \\
\oplus A_{2}^{k_{2}}
\end{array}, M_{1}^{l_{1}} M_{2}^{l_{2}}\right), Y^{n}\right) \notin T_{\eta_{5}(\eta)}\left(V_{1} \oplus V_{2}, Y\right)\right\}\right) \\
\leq & \left.P\left(\left(S_{j}^{n}, V_{j}^{n}\left(A_{j}^{k_{j}}, M_{j}^{l_{j}}\right): j=1,2\right) \in T_{\frac{\eta_{5}(\eta)}{2}}(\boldsymbol{S}, \boldsymbol{V}),\left(V \underset{\oplus A_{2}^{k_{2}}}{A_{1}^{k_{1}} 0^{k_{+}}}, M_{1}^{l_{1}} M_{2}^{l_{2}}\right), Y^{n}\right) \notin T_{\eta_{5}(\eta)}\left(V_{1} \oplus V_{2}, Y\right)\right),
\end{aligned}
$$

and the above two observations imply that $\left(V\left(A_{1}^{k_{1}} 0^{k_{+}} \oplus A_{2}^{k_{2}}, M_{1}^{l_{1}} M_{2}^{l_{2}}\right), \boldsymbol{X}^{n}, Y^{n}\right)$ is distributed according to $\prod_{t=1}^{n} P\left(V_{1} \oplus V_{2}=v_{t}, \boldsymbol{X}=\boldsymbol{x}_{t}, Y_{t}=y_{t} \mid \boldsymbol{S}_{t}=\boldsymbol{s}_{t}, \boldsymbol{V}_{t}=\boldsymbol{v}_{t}\right)$. Lemma 4 guarantees the existence of $N_{5}(\eta) \in \mathbb{N}$, such that for all $n \geq N_{5}(\eta)$, the term on the right hand side of 35 is bounded from above by $\frac{\eta}{8}$. Therefore, for all $n \geq N_{5}(\eta)$

$$
P\left(\left(\epsilon_{1} \cup \epsilon_{2} \cup \epsilon_{3}\right)^{c} \cap \epsilon_{4}^{c}\right) \leq \frac{\eta}{8} .
$$

It remains to provide an upper bound on $P\left(\left(\epsilon_{1} \cup \epsilon_{21} \cup \epsilon_{22} \cup \epsilon_{4}^{c}\right)^{c} \cap \epsilon_{5}\right)$. In appendix C, we prove the existence of $N_{6}(\eta) \in \mathbb{N}$ such that $P\left(\epsilon_{5}\right) \leq \exp \left\{-n\left(3 \eta_{5}(\eta)-\eta_{2}(\eta)-\eta_{3}(\eta)\right)\right\}$ for all $n \geq \max \left\{N_{1}(\eta), N_{6}(\eta)\right\}$. The informed reader will recognize that deriving an upper bound on $P\left(\epsilon_{5}\right)$ will involve proving statistical independence of the pair $\left(C_{j}\left(M_{j}^{l_{j}}\right): j=1,2\right)$ of cosets corresponding to the legitimate message pair $M_{j}^{l}$ 
and any codeword $V^{n}\left(\hat{a}^{k}, \hat{m}^{l}\right)$ corresponding to a competing message pair $\hat{m}^{l} \neq M^{l}$. This is considerably simple for a coding technique based on classical unstructured codes wherein codebooks and codewords in every codebook are independent. The coding technique proposed herein involves correlated codebooks - the first $k_{1}$ rows of $G_{I_{j}}: j=1,2$ are identica ${ }^{11}$ - and codewords in each codebook are correlated.

To conclude, we put together the upper bounds derived on the probability of events that comprise the error event. For $n \geq N_{2}(\eta), P\left(\epsilon_{1}\right) \leq \frac{\eta}{8}$. In 25 , we proved $P\left(\epsilon_{1 j}^{c} \cap \epsilon_{2 j}\right) \leq \exp \left\{-n\left(\eta_{1}(\eta)-\frac{3 \eta_{4}(\eta)}{2}\right)\right\}$ for all $n \geq N_{3}(\eta)$. Combining (32) and (35), we have

$$
P\left(\left(\epsilon_{1} \cup \epsilon_{2}\right)^{c} \cap \epsilon_{4}^{c}\right) \leq \exp \left\{-n\left(\lambda \eta_{5}^{2}(\eta)-4 \eta_{1}(\eta)-4 \eta_{4}(\eta)\right)\right\}+\frac{\eta}{8}
$$

for all $n \geq \max \left\{N_{1}(\eta), N_{4}(\eta), N_{5}(\eta)\right\}$. And finally $P\left(\epsilon_{5}\right) \leq \exp \left\{-n\left(\eta_{2}(\eta)+\eta_{3}(\eta)-3 \eta_{5}(\eta)\right)\right\}$ for all $n \geq \max \left\{N_{1}(\eta), N_{6}(\eta)\right\}$ follows from 69. By choosing

$$
\eta_{2}(\eta)=\eta_{3}(\eta)=\frac{\eta}{16}, \eta_{5}(\eta)=\frac{\eta}{48}, \eta_{1}(\eta)=\min \left\{\frac{\eta}{16}, \frac{\lambda \eta_{5}^{2}(\eta)}{10}\right\} \text { and } \eta_{4}(\eta)=\frac{\eta_{1}(\eta)}{4}
$$

it can be verified that for $n \geq \bar{N}(\eta):=\max \left\{N_{i}(\eta): i \in[6]\right\}$,

- $2 \eta_{1}(\eta)+2 \eta_{3}(\eta)<\frac{\eta}{2}$ and thus $\frac{l_{2} \log \pi}{n} \geq R_{2}-\frac{\eta}{2}$ from 23 ,

- $\eta_{2}(\eta)<\frac{\eta}{2}$ and thus $\frac{l_{1} \log \pi}{n}>R_{1}-\frac{\eta}{2}$ from 21 ,

- $\eta_{1}(\eta)-\frac{3 \eta_{4}(\eta)}{2}=\frac{5 \eta_{1}(\eta)}{8}$ and thus $P\left(\epsilon_{1 j}^{c} \cap \epsilon_{2 j}\right) \leq \exp \left\{-n\left(\frac{5 \eta_{1}(\eta)}{8}\right)\right\}$,

- $\lambda \eta_{5}^{2}(\eta)-4 \eta_{1}(\eta)-4 \eta_{4}(\eta) \geq \frac{\lambda \eta_{5}^{2}(\eta)}{2}$ and thus $P\left(\left(\epsilon_{1} \cup \epsilon_{2}\right)^{c} \cap \epsilon_{4}^{c}\right) \leq \exp \left\{-n\left(\frac{\lambda \eta_{5}^{2}(\eta)}{2}\right)\right\}+\frac{\eta}{8}$, and

- $\eta_{2}(\eta)+\eta_{3}(\eta)-3 \eta_{5}(\eta)=\frac{\eta}{16}$ and therefore $P\left(\epsilon_{5}\right) \leq \exp \left\{-n\left(\frac{\eta}{16}\right)\right\}$.

For $n \geq \bar{N}(\eta), P\left(\epsilon_{1}\right)+P\left(\epsilon_{11}^{c} \cap \epsilon_{21}\right)+P\left(\epsilon_{12}^{c} \cap \epsilon_{22}\right)+P\left(\left(\epsilon_{1} \cup \epsilon_{21} \cup \epsilon_{22}\right)^{c} \cap \epsilon_{4}^{c}\right)+P\left(\epsilon_{5}\right) \leq \frac{\eta}{4}+3 \exp \left\{-n\left(\frac{5 \eta_{1}}{8}\right)\right\}$. Thus for $n \geq N(\eta):=\max \left\{\bar{N}(\eta), \frac{1}{\eta_{1}(\eta)} \log \left\lceil\frac{4}{\eta}\right\rceil\right\}$, the error event has probability at most $\eta$.

We conclude this section with two remarks.

Remark 2: For BDD-MAC described in section VI-B, $\beta_{f}(\boldsymbol{\tau})=\mathbb{C}(\boldsymbol{\tau})$. Indeed, the test channel $p_{\boldsymbol{V} \boldsymbol{S} \boldsymbol{X} Y} \in$ $\mathbb{D}_{f}(\boldsymbol{\tau})$ defined as $p_{\boldsymbol{V} \boldsymbol{S} \boldsymbol{X}}=\prod_{j=1}^{2} p_{V_{j} S_{j} X_{j}}$ where $V_{j}$ takes values over $\mathcal{V}_{j}=\{0,1\}$ with

$$
p_{V_{j}, X_{j} \mid S_{j}}\left(x_{j} \oplus_{2} s_{j}, x_{j} \mid s_{j}\right)= \begin{cases}1-\tau & \text { if } x_{j}=0 \\ \tau & \text { otherwise }\end{cases}
$$

for each $j=1,2$ and $s_{j} \in\{0,1\}$ achieves $\mathbb{C}(\boldsymbol{\tau})=\left\{\left(R_{1}, R_{2}\right): R_{1}+R_{2} \leq h_{b}(\tau)\right\}$.

We have thus presented a coding technique based on decoding the sum of codewords chosen by the encoders and analyzed the same to derive an achievable rate region for an arbitrary MAC-DSTx. One

\footnotetext{
${ }^{11}$ If $H\left(V_{1} \mid S_{1}\right)=H\left(V_{2} \mid S_{2}\right)$, users 1 and 2 share the same generator matrix $G_{I}$. Indeed, channel codes of users' 1 and 2 are partitioned into cosets of the same linear code.
} 
might attempt a generalization of PZ-technique along the lines of modulo lattice transformation proposed by Haim, Kochman and Erez [31]. The rate region proposed herein subsumes that achievable through modulo-lattice transformation using test channels identified through the virtual channel in a natural way.

\section{Examples}

A key element of the coding framework proposed herein lies in characterizing achievable rate regions for arbitrary test channels, i.e., test channels that are not restricted to be uniform or additive in nature using structured codes.

A few remarks on our study of the following examples are in order. The examples needing to be nonadditive lends it considerably hard to provide analytical upper bounds for the rate region achievable using unstructured codes ${ }^{12}$ We therefore resort to computation. It can be noted that the problem of computing the sum rate bound achievable using unstructured codes is a non-convex optimization problem. The only approach is direct enumeration, i.e., sampling the probability matrix of the auxiliary random variables ${ }^{13}$ Sampling the probability matrix with any reasonable step size beyond the auxiliary alphabets of size 2 is infeasible with currently available computation resources. The sum rate bound for the unstructured coding technique projected below is therefore obtained through computation involving binary auxiliary alphabet sets followed by convexification (time sharing between different costs). The resulting space of probability distributions that respect the cost constraints is sampled with a step size of 0.015 in each dimension. The resulting bound on the sum rate achievable using unstructured codes (without time sharing) is marked with blue crosses (denoted $\alpha$ in the legend) in the plots. The resulting upper bound is obtained as an upper convex envelope. Similarly, sum rate achievable using nested coset codes is marked with red circles (denoted $\beta$ in the legend) in the plots.

For examples 1 and 2 , we assume the alphabet sets to be binary $\mathcal{S}_{j}=\mathcal{X}_{j}=\{0,1\}, j=1,2$, (ii) uniform and independent states, i.e., $W_{\boldsymbol{S}}(\boldsymbol{s})=\frac{1}{4}$ for all $s \in \mathcal{S}$, (iii) a Hamming cost function $\kappa_{j}\left(1, s_{j}\right)=1$ and $\kappa_{j}\left(0, s_{j}\right)=0$ for any $s_{j} \in \mathcal{S}_{j}, j=1,2$.

Example 1: Let $Y=\left(X_{1} \vee S_{1}\right) \oplus\left(X_{2} \vee S_{2}\right)$, where $\vee$ denotes logical OR operator. Having studied the BDD-MAC it is natural to conjecture that the test channel that optimizes the sum rate achievable using linear codes to be $p_{U_{j} X_{j} \mid S_{j}}(0,0 \mid 0)=1-2 \tau, p_{U_{j} X_{j} \mid S_{j}}(1,1 \mid 0)=2 \tau, p_{U_{j} X_{j} \mid S_{j}}(1,0 \mid 1)=1$, for $j=1,2$

\footnotetext{
${ }^{12}$ We recognize that the analytical upper bound derived in [4] is a key element of the findings therein.

${ }^{13}$ This holds even for the case of multiple access without states for which a computable characterization of the capacity region is known.
} 


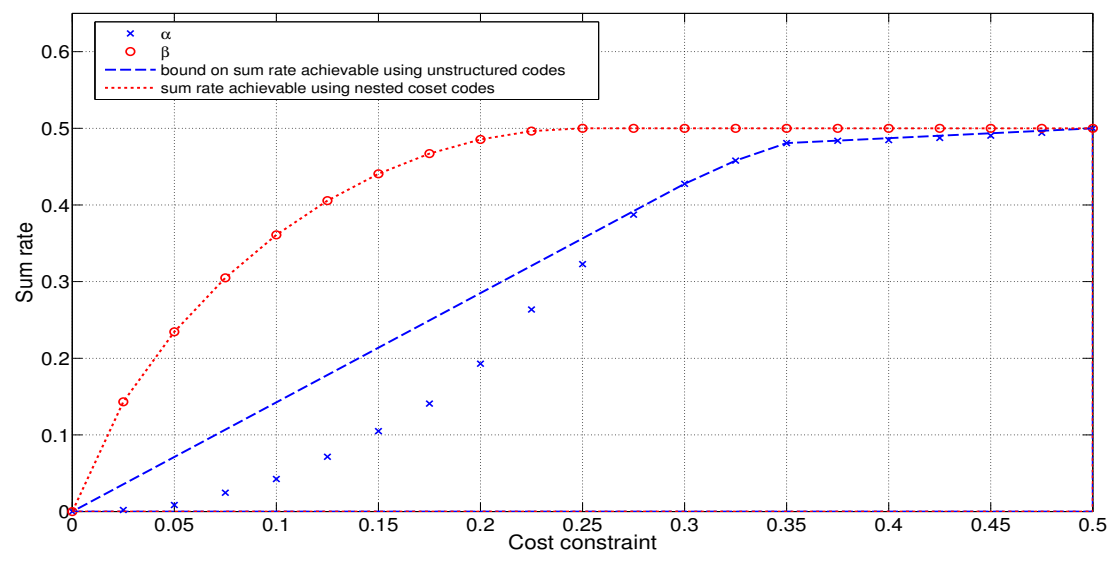

Fig. 1. Bounds on sum rate for example 1

when the cost constraint $\tau \in\left[0, \frac{1}{4}\right]$. Indeed, our numerical computation asserts this. In other words, the sum rate achievable using linear codes for a cost $\tau \in\left(0, \frac{1}{4}\right)$ is $\frac{h_{b}(2 \tau)}{2}$ and 0.5 for $\tau \in[0.25,0.5]$. The sum rate achievable using unstructured codes and nested coset codes are plotted in figure 1. We highlight significant gains achievable using nested coset codes.

A preliminary look at this channel may lead the reader to conclude that PZ-technique appropriately modified can achieve the same sum rate as that achievable using nested coset codes, since the above test channel is additive, i.e., $U_{j}=S_{j} \oplus X_{j}$ for $j=1,2$ and $Y=U_{1} \oplus U_{2}$. However, a careful analysis will reveal the significance of the coding framework proposed herein. The induced pmf on $U_{j}, p_{U_{j}}(1)=\frac{1}{2}+2 \tau$ for $\tau \in\left(0, \frac{1}{4}\right)$ is not uniform, and the PZ-technique of choosing a codeword in the indexed bin with an average Hamming distance of $\tau$ does not yield the sum rate guaranteed by nested coset codes. Nesting of codes enables achieving non-uniform distributions that are necessary as exemplified herein.

Example 2: The channel transition matrix is given in table 1) 1) An upper bound on sum rate achievable using unstructured codes and 2) sum rate achievable using nested coset codes are plotted in figure 2 . This channel is obtained by randomly perturbing the BDD-MAC ${ }^{14}$ In the space of channel transition probability matrices, this channel is in a neighborhood of the BDD-MAC. Since the rate regions are continuous functions over this space of channels, the coding technique proposed herein outperforms unstructured coding technique in this neighborhood. This example validates the same. As in the previous example, we note that the optimizing distribution of the auxiliary random variables is non-uniform

\footnotetext{
${ }^{14}$ The reader is referred to [32. Section VI.C] wherein we have presented results for a few more channels obtained by a random perturbation of the BDD-MAC.
} 


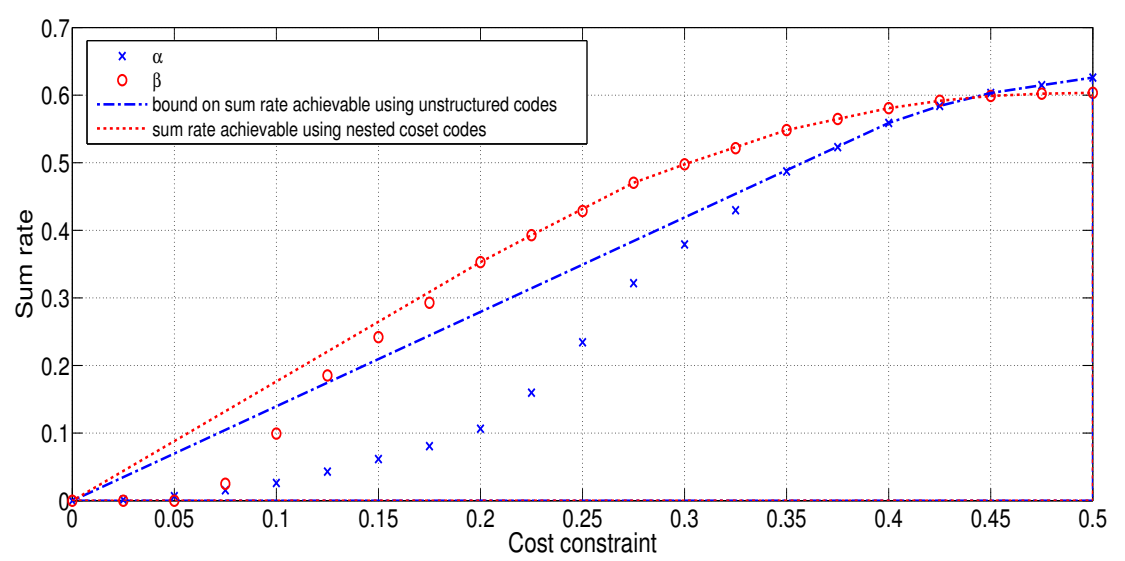

Fig. 2. Bounds on sum rate for example 2

\begin{tabular}{|c|c|c|c|c|c|c|c|}
\hline$S_{2} X_{2} S_{1} X_{1}$ & $W_{Y \mid S X^{(0 \mid \cdot)}}$ & $S_{2} X_{2} S_{1} X_{1}$ & $W_{\left.Y \mid S X^{(0 \mid} \cdot\right)}$ & $S_{2} X_{2} S_{1} X_{1}$ & $W_{\left.Y \mid S X^{(0 \mid} \cdot\right)}$ & $S_{2} X_{2} S_{1} X_{1}$ & $W_{Y \mid S X^{(0 \mid \cdot)}}$ \\
\hline \hline 0000 & 0.92 & 1000 & 0.07 & 0100 & 0.10 & 1100 & 0.88 \\
\hline 0001 & 0.08 & 1001 & 0.92 & 0101 & 0.92 & 1101 & 0.08 \\
\hline 0010 & 0.06 & 1010 & 0.96 & 0110 & 0.95 & 1110 & 0.11 \\
\hline 0011 & 0.94 & 1011 & 0.10 & 0111 & 0.06 & 1111 & 0.91 \\
\hline
\end{tabular}

TABLE I

CHANNEL TRANSITION MATRIX EXAMPLE2

for certain cost values. Furthermore, note that $\beta_{f}(\boldsymbol{\tau})$ does not contain $\alpha(\boldsymbol{\tau})$ and therefore it helps to incorporate both unstructured and structured coding techniques as will be studied in the following section.

Example 3: Consider the channel $Y=\left(S_{1} \oplus X_{1}\right) \vee\left(S_{2} \oplus X_{2}\right)$. Observe that the information available at the encoders is fused through a logical OR operation by the channel. Moreover, $\left(U_{1}, U_{2}\right)-U_{1} \oplus_{3} U_{2}-$ $U_{1} \vee U_{2}$ is a Markov chain and hence, although channel input, state and output alphabets are binary, we expect that for certain choice of auxiliary distributions, the sum rate achievable using codes over $\mathcal{F}_{3}$ is larger than that achievable using unstructured codes. Through an exhaustive search, we have identified such distributions, an example of which is given in table III.

For the distribution in table $\Pi$, the rate achievable using nested coset codes over $\mathcal{F}_{3}$ is 0.0017 , while that achievable using unstructured code is negative. For an appropriate choice of cost function, the above might be the optimizing distribution for the unstructured coding scheme thus resulting in larger sum rate using nested coset codes over $\mathcal{F}_{3}$. We do not as of yet have a precise analytical characterization of such 


\begin{tabular}{|c|c|c|c|c|c|c|c|}
\hline$U_{1} S_{1} X_{1}$ & $p_{U_{1} S_{1} X_{1}}$ & $U_{1} S_{1} X_{1}$ & $p_{U_{1} S_{1} X_{1}}$ & $U_{2} S_{2} X_{2}$ & $p_{U_{2} S_{2} X_{2}}$ & $U_{2} S_{2} X_{2}$ & $p_{U_{2} S_{2} X_{2}}$ \\
\hline \hline 000 & 0.1472 & 101 & 0.3528 & 000 & 0.1472 & 101 & 0.3528 \\
\hline 011 & 0.50 & & & 011 & 0.50 & & \\
\hline
\end{tabular}

TABLE II

TEST CHANNEL FOR EXAMPLE 3 FOR WHICH NESTED COSET CODE OVER $\mathcal{F}_{3}$ PERFORMS BETTER THAN UNSTRUCTURED

CODE

a cost function ${ }^{15}$ and we are in pursuit of the same. Nevertheless, the above lends credence to the use of nested coset codes for arbitrary channels.

\section{Stage II: COMBINING UNSTRUCTURED AND STRUCTURED CODING TECHNIQUES}

In this section, we put together the techniques of unstructured and structured random coding to derive a larger achievable rate region for a general MAC-DSTx. Our approach is similar to that proposed by Ahlswede and Han [9, Section VI] for the problem of reconstructing mod-2 sum of distributed binary sources. We begin with a characterization of valid test channels.

Definition 11: Let $\mathbb{D}_{s f}(\boldsymbol{\tau}) \subseteq \mathbb{D}(\boldsymbol{\tau})$ be the collection of distributions $p_{\boldsymbol{U} V \boldsymbol{S} \boldsymbol{X} Y}$ on $(\mathcal{U} \times \mathcal{V})^{2} \times \mathcal{S} \times \mathcal{X} \times \mathcal{Y}$ where $\mathcal{U}$ is a finite set and $\mathcal{V}$ is a finite field. For $p_{\boldsymbol{U} V \boldsymbol{S} \boldsymbol{X} Y} \in \mathbb{D}_{s f}(\boldsymbol{\tau})$, let $\mathcal{R}_{s f}\left(p_{\boldsymbol{U} \boldsymbol{V} \boldsymbol{X} \boldsymbol{S} Y}\right)$ be defined as the set of rate pairs $\left(R_{1}, R_{2}\right) \in[0, \infty)^{2}$ that satisfy

$$
\begin{gathered}
R_{1} \leq I\left(U_{1} ; U_{2} Y\right)-I\left(U_{1} ; S_{1}\right)+\min \left\{H\left(V_{1} \mid U_{1}, S_{1}\right), H\left(V_{2} \mid U_{2}, S_{2}\right)\right\}-H\left(V_{1} \oplus V_{2} \mid U_{1}, U_{2}, Y\right), \\
R_{2} \leq I\left(U_{2} ; U_{1} Y\right)-I\left(U_{2} ; S_{2}\right)+\min \left\{H\left(V_{1} \mid U_{1}, S_{1}\right), H\left(V_{2} \mid U_{2}, S_{2}\right)\right\}-H\left(V_{1} \oplus V_{2} \mid U_{1}, U_{2}, Y\right), \\
R_{1}+R_{2} \leq I\left(U_{1} U_{2} ; Y\right)+I\left(U_{1} ; U_{2}\right)-\sum_{j=1}^{2} I\left(U_{j} ; S_{j}\right)+\min \left\{H\left(V_{1} \mid U_{1}, S_{1}\right), H\left(V_{2} \mid U_{2}, S_{2}\right)\right\} \\
-H\left(V_{1} \oplus V_{2} \mid U_{1}, U_{2}, Y\right),
\end{gathered}
$$

where $\oplus$ is addition in $\mathcal{V}$. Let

$$
\mathcal{R}_{s f}(\boldsymbol{\tau}):=\operatorname{cocl}\left(\bigcup_{p_{\boldsymbol{U} V \boldsymbol{X} S Y} \in \mathbb{D}_{s f}(\boldsymbol{\tau})} \mathcal{R}_{s f}\left(p_{\boldsymbol{U} \boldsymbol{V} \boldsymbol{X} S Y}\right)\right)
$$

Theorem 5: $\mathcal{R}_{s f}(\boldsymbol{\tau}) \subseteq \mathcal{C}(\boldsymbol{\tau})$.

Remark 3: $\alpha(\boldsymbol{\tau}) \subsetneq \mathcal{R}_{s f}(\boldsymbol{\tau})$.

\footnotetext{
${ }^{15}$ Such a characterization of cost function is available for point-to-point channels with state available at both encoder and decoder [22], [33], [34].
} 
Proof: Achievability of $\mathcal{R}_{s f}(\boldsymbol{\tau})$ is proved by gluing together unstructured and structured coding techniques. Each encoder splits it's message $M_{j}$ into two parts $M_{j, 1}$ and $M_{j}^{l_{j}} . M_{j, 1}$ is communicated to the decoder using an unstructured random code built over $\mathcal{U}^{n} . M_{j}^{l_{j}}$ is communicated to the decoder using a nested coset code identical to that proposed in proof of theorem 4 . With regard to nested coset codes, we employ the notation proposed in the proof of theorem 4 and do not restate the same.

Encoder $j$ is provided a codebook built over $\mathcal{U}^{n}$ that contains $2^{n \bar{R}_{j}}$ bins each with $2^{n B_{j}}$ codewords. For $1 \leq b_{j} \leq 2^{n B_{j}}$, let $u_{j}\left(r_{j}, b_{j}\right)$ denote a generic codeword in bin $r_{j}\left(1 \leq r_{j} \leq 2^{n \bar{R}_{j}}\right)$. Encoder $j$ is also provided with the nested coset code $\lambda_{O_{j} / I}$. Without loss of generality, we assume $M_{j}^{l_{j}} \in$ $\mathcal{V}^{l_{j}}$. Encoder $j$ observes state sequence $S_{j}^{n}$ and declares error if $S_{j}^{n} \notin T_{\frac{\delta}{8}}\left(W_{S_{j}}\right)$. Otherwise it looks for a pair $\left(u_{j}^{n}\left(M_{j, 1}, b_{j}\right), v^{n}\left(a^{k}, M_{j}^{l_{j}}\right)\right) \in T_{\frac{\delta}{4}}\left(U_{j} V_{j} \mid S_{j}^{n}\right)$. If it finds at least one such pair, one of them say, $\left(u_{j}^{n}\left(M_{j, 1}, b_{j}\right), v^{n}\left(a^{k}, M_{j}^{l_{j}}\right)\right)$ is chosen uniformly at random and $e_{j}^{n}\left(M_{j}, S_{j}^{n}\right)$ is transmitted, where $e_{j}^{n}\left(M_{j}, S_{j}^{n}\right)$ is a function of $u_{j}^{n}\left(M_{j, 1}, b_{j}\right), v^{n}\left(a^{k}, M_{j}^{l_{j}}\right), S_{j}^{n}$ that is determined upfront. Otherwise, an error is declared.

We now specify the decoding rule. The decoder receives $Y^{n}$ and declares error if $Y^{n} \notin T_{\frac{\delta}{2}}(Y)$. Otherwise, decoding is performed in two stages. In the first stage it lists all codewords $\left(u_{j}^{n}\left(m_{j, 1}, b_{j}\right): j=\right.$ $1,2) \in T_{\delta}^{n}\left(U_{1}, U_{2} \mid y^{n}\right)$. If it finds exactly one such pair, say $\left(u_{j}^{n}\left(m_{j, 1}, b_{j}\right): j=1,2\right)$, then the decoding proceeds to the next stage. Otherwise, an error is declared and decoding halts. In the second stage, the decoder looks for all codewords $v^{n}\left(a^{k}, \mathbf{m}^{\mathbf{l}}\right) \in \lambda_{O}$ such that $\left(u_{j}^{n}\left(m_{j, 1}, b_{j}\right): j=1,2, v^{n}\left(a^{k}, \mathbf{m}^{\mathbf{l}}\right), Y^{n}\right) \in$ $T_{\delta}^{n}\left(U, V_{1} \oplus V_{2}, Y\right)$. If it finds all such codewords in a unique bin, say corresponding to $\mathbf{m}^{\mathbf{l}}$, then it declares $m_{j, 1}, m_{j}^{l_{j}}: j=1,2$ as the decoded pair of messages. Otherwise, an error is declared. We derive an upper bound on probability of error by averaging the error probability over the ensemble codes. A pmf is induced over the ensemble of codes by letting $U_{j}^{n}\left(r_{j}, b_{j}\right): 1 \leq r_{j \leq 2^{n \bar{R}_{j}}}, 1 \leq b_{j} \leq 2^{n B_{j}}, j=1,2$ be mutually independent and distributed according to $\prod_{t=1}^{n} p_{U_{j}}$. The pmf induced on the ensemble of nested coset codes is identical to that in proof of theorem 4 Moreover, $\left(G_{I}, G_{O_{j} / I}, B_{j}^{n}: j=1,2\right)$ is independent of the unstructured random code on $\mathcal{U}^{n}$. Analyzing the error events, we obtain the following sufficient conditions for the average probability of error to decay exponentially.

$$
\begin{array}{rc}
B_{1} \geq I\left(U_{1} ; S_{1}\right) & B_{2} \geq I\left(U_{2} ; S_{2}\right) \\
\bar{R}_{1}+B_{1} \leq I\left(U_{1} ; U_{2} Y\right) & \bar{R}_{2}+B_{2} \leq I\left(U_{2} ; U_{1} Y\right) \\
\frac{k}{n} \geq 1-H\left(V_{1} \mid U_{1} S_{1}\right) & \frac{k}{n} \geq 1-H\left(V_{2} \mid U_{2} S_{2}\right) \\
\sum_{j=1}^{2} \bar{R}_{j}+B_{j} \leq I(\boldsymbol{U} ; Y)+I\left(U_{1} ; U_{2}\right) & \frac{l_{1}+l_{2}}{n} \leq 1-H\left(V_{1}+V_{2} \mid \boldsymbol{U} Y\right) .
\end{array}
$$


For each $j=1,2$, substituting $R_{j}-\frac{l_{j}}{n}$ for $\bar{R}_{j}$ in the above bounds and eliminating $B_{j}, \frac{k}{n}, \frac{l_{j}}{n}: j=1,2$ using the technique of Fourier-Motzkin [21, Appendix D], $\mathcal{R}_{s f}(\boldsymbol{\tau})$ is proved achievable.

Remark 4: The above rate region is obtained by analyzing sequential typicality encoding and decoding, i.e., encoding and decoding of unstructured codes precedes that of structured codes. The informed reader will recognize that performing joint typicality encoding and decoding of unstructured and structured codes might enlarge the achievable rate region. While this might be true, Fourier-Motzkin elimination of the resulting bounds does not yield a compact description of the resulting achievable rate region. We therefore chose to present the above rate region.

We conclude with an illustrative example.

Example 4: For $j=1,2$, let $\mathcal{S}_{j}=\mathcal{X}_{j}=\mathcal{Y}=\{0,1\}$. The channel transition is described as $W_{Y \mid \boldsymbol{X} \boldsymbol{S}}(y \mid \boldsymbol{x}, \boldsymbol{s})=W_{Y \mid g(\boldsymbol{X}, \boldsymbol{S})}^{*}(y \mid g(\boldsymbol{x}, \boldsymbol{s}))$, where $g(\boldsymbol{x}, \boldsymbol{s})=\left[\left(s_{2} \wedge \overline{x_{2}}\right) \wedge\left(\overline{s_{1}} \vee x_{1}\right)\right] \vee\left[\left(s_{1} \wedge \overline{x_{1}}\right) \wedge\left(\overline{s_{2}} \vee x_{2}\right)\right]$, $\wedge$ denotes logical AND, and $W_{Y \mid g(\boldsymbol{X}, \boldsymbol{S})}^{*}(1 \mid 0)=0.02, W_{Y \mid g(\boldsymbol{X}, \boldsymbol{S})}^{*}(0 \mid 1)=0.04$. The function $g(\cdot, \cdot)$ can be alternatively described as $g(\boldsymbol{X}, \boldsymbol{S})=\left[S_{1} \wedge\left(S_{1} \oplus X_{1}\right)\right] \oplus\left[S_{2} \wedge\left(S_{2} \oplus X_{2}\right)\right]$.

This channel is inspired by Blackwell's broadcast channel and in particular the coding technique proposed by Gelfand [35] ${ }^{16}$ The bounds on the sum rate achievable with unstructured and nested coset codes are plotted in figure 3 . The above plots unequivocally indicate $\mathcal{R}_{s f}(\tau)$ to be strictly larger than $\alpha(\boldsymbol{\tau}) \cup \beta_{f}(\boldsymbol{\tau})$ and in particular either one of $\alpha(\boldsymbol{\tau}), \beta_{f}(\boldsymbol{\tau})$. It is therefore desirable to compute $\mathcal{R}_{s f}(\boldsymbol{\tau})$, however the presence of two additional auxiliary random variables lends computation infeasible with current computational resources. We remark that the structure of this example enables us to argue the strict containment $\alpha(\boldsymbol{\tau}) \cup \beta_{f}(\boldsymbol{\tau}) \subsetneq \mathcal{R}_{s f}(\boldsymbol{\tau})$ in spite of not being able to compute $\mathcal{R}_{s f}(\boldsymbol{\tau})$.

\section{Stage III: Achievable Rate Region using codes over Abelian groups}

Consider a quaternary doubly dirty MAC-DSTx (QDD-MAC), with $\mathcal{S}_{j}=\mathcal{X}_{j}=\mathcal{Y}=\{0,1,2,3\}$, $j=1,2$. The state sequences are independent and uniformly distributed, i.e., $W_{\boldsymbol{S}}(\boldsymbol{s})=\frac{1}{16}$ for all $s \in \mathcal{S}$. The channel transition is described by the relation $Y=X_{1} \oplus S_{1} \nLeftarrow X_{2} \diamond S_{2}$, where $\diamond$ denotes addition mod-4. All nonzero symbols have equal cost, i.e., $\kappa_{j}\left(x, s_{j}\right)=1$ for all $x \in\{1,2,3\}$ and $\kappa_{j}\left(0, s_{j}\right)=0$ for all $s_{j} \in \mathcal{S}_{j}, j=1,2$ and the input is subject to a symmetric cost constraint $\tau=(\tau, \tau)$.

What would be the achievable rate region for QDD-MAC using unstructured codes? It is natural to

\footnotetext{
${ }^{16}$ Analogous to the defect masking the written bits, here the states mask the corresponding channel.
} 


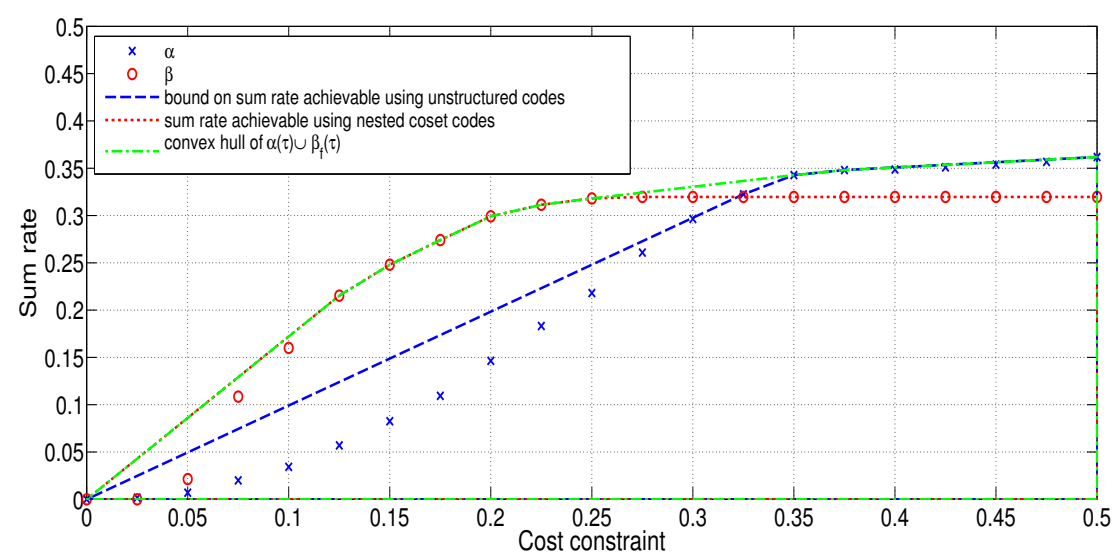

Fig. 3. Bounds on sum rate for example 4

guess the optimizing test channel to be

$$
p_{X_{j} V_{j} \mid S_{j}}\left(x_{j}, x_{j} \uplus s_{j} \mid s_{j}\right)= \begin{cases}1-\tau & \text { for } x_{j}=0 \\ \frac{\tau}{3} & \text { otherwise. }\end{cases}
$$

In appendix D of [32], with the aid of numerical computation, we argue that this is indeed the case. The sum rate achievable using unstructured codes can be evaluated to be the upper convex envelope of the function $\alpha:\left[0, \frac{3}{4}\right] \rightarrow[0, \infty)$ defined as $\left.\alpha(\tau)=\max \left\{-2 \tau \log \left(\frac{\tau}{3}\right)-2(1-\tau) \log (1-\tau)\right)-2,0\right\}$. Since 4 is a prime power, there exists a unique field $\mathcal{F}_{4}$ of cardinality 4 . Do nested coset codes built over $\mathcal{F}_{4}$ achieve a larger sum rate?

We are unable to characterize the sum rate achievable using nested coset codes and the dimensionality of the space of probability distributions lends computation infeasible. We conjecture that the above test channel optimizes the sum rate achievable using nested coset codes. In any case, computing the sum rate achievable using nested coset codes for the above test channel is instructive. It can be verified that the sum rate achievable using the above test channel with nested coset codes is the upper convex envelope of the function $\beta_{f}:\left[0, \frac{3}{4}\right] \rightarrow[0, \infty)$ defined as $\left.\beta_{f}(\tau)=\max \left\{-\tau \log \left(\frac{\tau}{3}\right)-(1-\tau) \log (1-\tau)\right)-\frac{1}{2}, 0\right\}$.

The sum rate achievable for the above test channel using unstructured and nested coset codes are plotted in figure 4. It is no surprise that nested coset codes perform poorly. The channel operation is not the field addition $\oplus_{4}$ in $\mathcal{F}_{4}$. Instead, $\diamond$ is the group addition ${ }^{17}$ in the Abelian group $\mathbb{Z}_{4}$. This suggests that we build codes over Abelian groups that are closed under group addition and decode the group sum

\footnotetext{
${ }^{17}$ We refer to group operation of an Abelian group as group addition.
} 


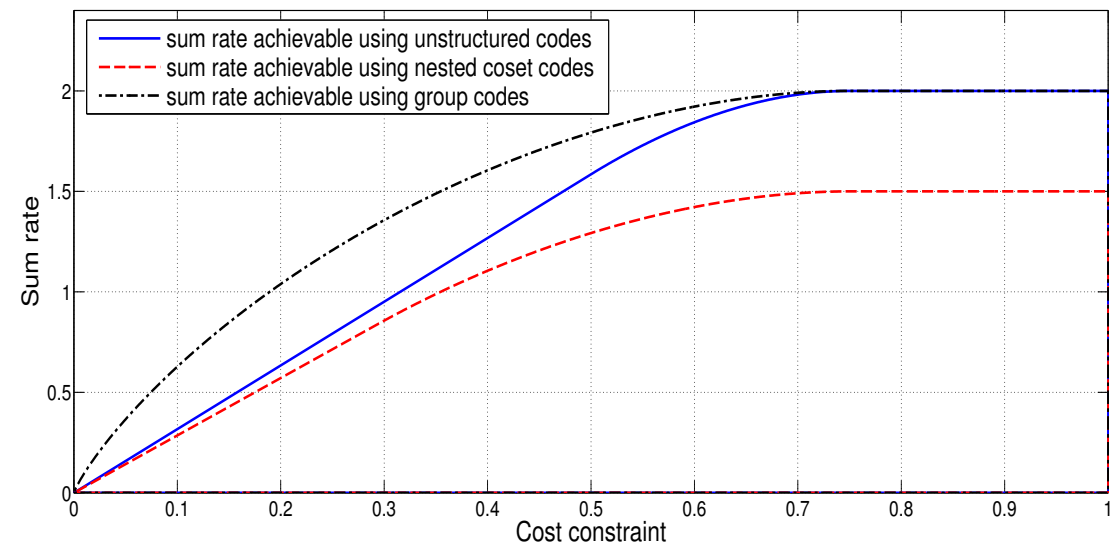

Fig. 4. Sum rate achievable using unstructured, nested coset and Abelian group codes for test channel 38

$\diamond$ of codewords.

Linear codes are kernels of field homomorphisms. This lends them the property of closure under field addition. We build Abelian group codes that are kernels of group homomorphisms. Abelian group codes are closed under group addition. As was proposed with nested coset codes, we employ bins of each user's code to be cosets of a common Abelian group code. The encoder chooses a codeword from the bin indexed by the message and the decoder attempts to localize the group sum of chosen codewords. The bins of each users' codebook is chosen such that the decoder can decode the pair of messages by identifying the group sum of transmitted codewords.

In the interest of brevity, we only describe the results and omit proofs. Recall that any Abelian group $\mathcal{V}$ can be decomposed as sum of $\mathbb{Z}_{p^{r}}$-cyclic groups, i.e.,

$$
\mathcal{V}=\bigoplus_{i=1}^{I} \mathbb{Z}_{p_{i}^{r_{i}}},
$$

where $p_{i}$ is a prime and $r_{i}$ is a positive integer for each $i=1, \cdots, I$. We therefore state our findings in two stages. The first stage, described in section VIII-A describes the coding technique and achievable rate region for a $\mathbb{Z}_{p^{r}}-$ group. This is extended to an arbitrary Abelian group in section VIII-B

\section{A. Achievable rate region for MAC-DSTx using group codes : The $\mathbb{Z}_{p^{r} \text {-case }}$}

In the discussion following proof of theorem 2 , we noted that if the auxiliary alphabet $\mathcal{V}$ is a field and the bins are constrained to be closed under field addition then with respect to a test channel $p_{V \mid S}$, the bins need to be of rate at least $\log |\mathcal{V}|-H(V \mid S)$. This enlargement of the bins was compensated by the ability 
to pack more bins. In particular, the rate of the composite code could be as large as $\log |\mathcal{V}|-H(V \mid Y)$ with respect to the induced distribution $p_{V \mid Y}$, and this enabled us to achieve the capacity of PTP-STx.

If the auxiliary alphabet $\mathcal{V}=\mathbb{Z}_{p^{r}}$ is an Abelian group of order $p^{r}$, and the bins are restricted to be closed under group addition, then with respect to a test channel $p_{V \mid S}$, using the results of [36], the bins have to be of rate at least

$$
\bar{I}_{s}^{\mathcal{V}}(V ; S)=\max _{\theta=1}^{r}\left[r \log p-\frac{r}{\theta} H\left([V]_{\theta} \mid S\right)\right]=\max _{\theta=1}^{r} \frac{r}{\theta} I\left([V]_{\theta} ; S\right),
$$

where $\mathcal{H}_{\theta}$ is the sub-group $p^{\theta} \mathbb{Z}_{p^{r}}$ and $[V]_{\theta}:=V \diamond \mathcal{H}_{\theta}$ is the random variable taking values from cosets of subgroup $\mathcal{H}_{\theta}$ of $\mathcal{V}$, denoted $\mathcal{H}_{\theta} \preceq \mathcal{V}$. We note that $\bar{I}_{s}^{\mathcal{V}}(V ; S) \geq \log q-H(V \mid S) \geq I(V ; S)$. The natural question to ask is whether we can pack sufficient number of bins to achieve capacity of PTP-STx. It turns out that if we constrain the composite code, i.e., the union of bins, to be a coset of a group code, then the rate of this union can be at most

$$
\bar{I}_{c}^{\mathcal{V}}(V ; Y)=\min _{\theta=0}^{r-1}\left[r \log p-\frac{r}{r-\theta} H\left(V \mid Y[V]_{\theta}\right)\right]=\min _{\theta=0}^{r-1} \frac{r}{r-\theta} I\left(V ; Y \mid[V]_{\theta}\right) .
$$

with respect to the induced distribution $p_{V \mid Y}$. Since $\log |\mathcal{V}|-H(V \mid Y)$ corresponds to $\theta=0$ in the above expression, $\bar{I}_{c}^{\mathcal{V}}(V ; Y)$ is in general smaller than $\log |\mathcal{V}|-H(V \mid Y)$. Therefore, $\bar{I}_{c}^{\mathcal{V}}(V ; Y)-\bar{I}_{s}^{\mathcal{V}}(V ; S)$ is in general strictly smaller than the capacity of PTP-STx, implying the constraint of closure under group addition results in a rate penalty. This indicates that the use of group codes will in general result in rate penalties for multi-terminal communication problems ${ }^{18}$

With the objective of increasing $\bar{I}_{c}^{\mathcal{V}}(V ; Y)$ and therefore minimizing the rate penalty, we take a closer look at the coding technique proposed in section VI-B. While we exploited the property of bins being closed under field addition, we did not need the union of bins to be a coset. We therefore relax this and only require the bins to have an algebraic structure, i.e., a coset of a group code, but the composite code of each user is not required to be a coset of a group code. While this relaxation does not yield gains in achievable rate for the field case, we do obtain larger achievable rates while coding over groups. In particular, the rate of the composite code, or the union of bins can be as large as $\log |\mathcal{V}|-H(V \mid Y)$ which is in general larger than $\bar{I}_{c}^{\mathcal{V}}(V ; Y)$. Therefore, if we were to communicate over a PTP-STx $\left(\mathcal{S}, W_{S}, \mathcal{X}, \kappa, \mathcal{Y}, W_{Y \mid X S}\right)$ using codes over an Abelian $\mathbb{Z}_{p^{r}}$-group $\mathcal{V}=\mathbb{Z}_{p^{r}}$ and we constrained the bins to be closed under group addition, then the test channel $p_{V S X Y} \in \overline{\mathbb{D}}(\tau)$ yields an achievable rate $\log |\mathcal{V}|-H(V \mid Y)-\left(\bar{I}_{s}^{\mathcal{V}}(V ; S)\right)=$

\footnotetext{
${ }^{18}$ The interested reader is referred to [37], [38], [39] for early work on rates achievable using group codes for point-to-point channels. [40] provides bounds on rates achievable using Abelian group codes for point-to-point source and channel coding problems.
} 
$\bar{H}_{s}^{\mathcal{V}}(V \mid S)-H(V \mid Y)$, where

$$
\bar{H}_{s}^{\mathcal{V}}(V \mid S)=\log |\mathcal{V}|-\bar{I}_{s}^{\mathcal{V}}(V ; S)
$$

is defined as source coding group entropy of group $\mathcal{V}=\mathbb{Z}_{p^{r}}$ and $\bar{H}_{s}^{\mathcal{V}}(V)=\bar{H}_{s}^{\mathcal{V}}(V \mid 0)$.

The diligent reader will now be able to characterize an achievable rate region for a MAC-DSTx based on group codes. As mentioned earlier, the encoding and decoding techniques are identical to that proposed in section VI-B except for group addition replacing field addition. Consider a distribution $p_{\boldsymbol{V} \boldsymbol{S} \boldsymbol{X} Y} \in \mathbb{D}(\tau)$ defined over $\mathcal{V}^{2} \times \mathcal{S} \times \mathcal{X} \times \mathcal{Y}$ where $\mathcal{V}$ is an Abelian group of order $p^{r}$. Cosets of a common group code is employed as bins of each user's code. Following an analysis similar to that performed in proof of theorem 4, one can prove the probability of the encoders not finding a codeword jointly typical with the state sequence decays exponentially with block length if the bins are of rate at least $\max \left\{\log |\mathcal{V}|-\bar{H}_{s}^{\mathcal{V}}\left(V_{j} \mid S_{j}\right): j=1,2\right\}$. The decoder decodes the group sum of chosen codewords from the group sum of the two users' codebooks. The codebooks of the two users are chosen to be union of arbitrary cosets of a common group code and therefore the the group sum of the two users codebooks will also be a union of arbitrary cosets of this group code. The probability of error at the decoders decays exponentially if the rate of the group sum of the two users' codebooks is at most $\log |\mathcal{V}|-H\left(V_{1} \nLeftarrow V_{2} \mid Y\right)$. We conclude that a rate pair $\left(R_{1}, R_{2}\right)$ is achievable if $R_{1}+R_{2} \leq \min \left\{\bar{H}_{s}^{\mathcal{V}}\left(V_{j} \mid S_{j}\right): j=1,2\right\}-H\left(V_{1} \nLeftarrow\right.$ $\left.V_{2} \mid Y\right)$. The following is a formal characterization of achievable rate region for MAC-DSTx using group codes over a $\mathbb{Z}_{p^{r}}$-group.

Definition 12: Let $\mathbb{D}_{s g}(\boldsymbol{\tau}) \subseteq \mathbb{D}(\boldsymbol{\tau})$ be the collection of distributions $p_{\boldsymbol{U} V \boldsymbol{V} \boldsymbol{X} Y}$ on $(\mathcal{U} \times \mathcal{V})^{2} \times \mathcal{S} \times \mathcal{X} \times \mathcal{Y}$ where $\mathcal{U}$ is a finite set and $\mathcal{V}$ is an Abelian group of order $p^{r}$, where $p$ is a prime. For $p_{\boldsymbol{U} \boldsymbol{V} \boldsymbol{S} \boldsymbol{X} Y} \in \mathbb{D}_{s g}(\boldsymbol{\tau})$, let $\mathcal{R}_{s g}\left(p_{\boldsymbol{U} \boldsymbol{V} \boldsymbol{S} \boldsymbol{X} Y}\right)$ be defined as the set of rate pairs $\left(R_{1}, R_{2}\right) \in[0, \infty)^{2}$ that satisfy

$$
\begin{gathered}
R_{1} \leq I\left(U_{1} ; U_{2} Y\right)-I\left(U_{1} ; S_{1}\right)+\min \left\{\bar{H}_{s}^{\mathcal{V}}\left(V_{1} \mid U_{1}, S_{1}\right), \bar{H}_{s}^{\mathcal{V}}\left(V_{2} \mid U_{2}, S_{2}\right)\right\}-H\left(V_{1} \diamond V_{2} \mid U_{1}, U_{2}, Y\right), \\
R_{2} \leq I\left(U_{2} ; U_{1} Y\right)-I\left(U_{2} ; S_{2}\right)+\min \left\{\bar{H}_{s}^{\mathcal{V}}\left(V_{1} \mid U_{1}, S_{1}\right), \bar{H}_{s}^{\mathcal{V}}\left(V_{2} \mid U_{2}, S_{2}\right)\right\}-H\left(V_{1} \oplus V_{2} \mid U_{1}, U_{2}, Y\right), \\
R_{1}+R_{2} \leq I\left(U_{1} U_{2} ; Y\right)+I\left(U_{1} ; U_{2}\right)-\sum_{j=1}^{2} I\left(U_{j} ; S_{j}\right)+\min \left\{\bar{H}_{s}^{\mathcal{V}}\left(V_{1} \mid U_{1}, S_{1}\right), \bar{H}_{s}^{\mathcal{V}}\left(V_{2} \mid U_{2}, S_{2}\right)\right\} \\
-H\left(V_{1} \nLeftarrow V_{2} \mid U_{1}, U_{2}, Y\right),
\end{gathered}
$$

where $\diamond$ denotes group addition in group $\mathcal{V}=\mathbb{Z}_{p^{r}}$, and

$$
\mathcal{R}_{s g}(\boldsymbol{\tau}):=\operatorname{cocl}\left(\bigcup_{p_{U V X S Y} \in \mathbb{D}_{s g}(\boldsymbol{\tau})} \mathcal{R}_{s g}\left(p_{\boldsymbol{U} \boldsymbol{V} \boldsymbol{X} \boldsymbol{S} Y}\right)\right)
$$

Theorem 6: $\mathcal{R}_{s g}(\boldsymbol{\tau}) \subseteq \mathbb{C}(\boldsymbol{\tau})$.

Example 5: Let us now compute the achievable rate region using group codes for QDD-MAC. We only compute $\mathcal{R}_{s g}\left(p_{\boldsymbol{U} \boldsymbol{V} \boldsymbol{X} \boldsymbol{S} Y}\right)$ where $\mathcal{U}=\phi$, the empty set and $\mathcal{V}=\{0,1,2,3\}$ and $p_{V S X}$ is given in 
38. $\mathcal{V}=\{0,1,2,3\}$ has two sub-groups - the group itself, $\{0,2\}$. It can be verified that

$$
\bar{I}_{s}^{\mathcal{U}}(U ; S)=\max \left\{\log _{2} 4-2 h_{b}\left(\frac{2 \tau}{3}\right), \log _{2} 4+\tau \log _{2}\left(\frac{\tau}{3}\right)+(1-\tau) \log _{2}(1-\tau)\right\}
$$

yielding $\mathcal{R}_{s g}\left(p_{\boldsymbol{U} \boldsymbol{V} \boldsymbol{X} \boldsymbol{S Y}}\right)=\left\{\left(R_{1}, R_{2}\right) \in[0, \infty)^{2}: R_{1}+R_{2} \leq\left|\beta_{g}(\tau)\right|^{+}\right\}$, where

$$
\beta_{g}(\tau)=\max \left\{\min \left\{-\tau \log _{2}\left(\frac{\tau}{3}\right)-(1-\tau) \log _{2}(1-\tau), 2 h_{b}\left(\frac{2 \tau}{3}\right)\right\}, 0\right\} .
$$

In figure 4, the sum rate achievable using group codes for the above test channel is plotted. We highlight significant gains achievable using group codes for QDD-MAC thus emphasizing the need to build codes with appropriate algebraic structure that matches the channel.

\section{B. Achievable rate region for MAC-DSTx using group codes: The general Abelian group}

We now let the auxiliary alphabet $\mathcal{V}$ be a general Abelian group and build group codes over $\mathcal{V}$ to enable the decoder to reconstruct the group sum of chosen codewords. The discussion in section VIII-A indicates that we only need to characterize the minimum rate of a bin in the code with respect to a generic test channel $p_{V \mid S}$ under the constraint that the bin has to be a coset of a group code. Essentially, this will involve characterizing fundamental group information theoretic quantity $\bar{I}_{s}^{\mathcal{V}}(V ; S)$ and the related source coding group entropy $\bar{H}_{s}^{\mathcal{V}}(V \mid S)$ in the context of a general Abelian group $\mathcal{V}$.

Let $\mathcal{V}$ be the Abelian group in 39]. Let $\theta=\left(\theta_{1}, \cdots, \theta_{r}\right)$ be such that $0 \leq \theta_{i} \leq r_{i}$ for $i=1,2, \cdots, I$ and let $\mathcal{H}_{\theta}$ be a subgroup of $\mathcal{V}$ defined as

$$
\mathcal{H}_{\theta}=\bigoplus_{i=1}^{I} p^{\theta_{i}} \mathbb{Z}_{p_{i}^{r_{i}}}
$$

and random variable $[V]_{\theta}$ taking values from cosets of $\mathcal{H}_{\theta}$ in $\mathcal{V}$ as $[V]_{\theta}=V \nLeftarrow \mathcal{H}_{\theta}$. If the state has a pmf $p_{S}$ and the bins over $\mathcal{V}$ are constrained to be cosets of a group code, then for a test channel $p_{V \mid S}$, the rate of a bin has to be at least

$$
\bar{I}_{s}^{\mathcal{V}}(V ; S):=\min _{\substack{w_{1}, \cdots, w_{I} \\ w_{1}+\cdots+w_{I}=1}} \max _{\substack{\mathcal{H} \prec \mathcal{V} \\ \mathcal{H} \neq \mathcal{V}}} \frac{1}{1-w_{\theta}} I\left([V]_{\theta} ; S\right)
$$

where

$$
w_{\theta}=\sum_{i=1}^{I} \frac{r_{i}-\theta_{i}}{r_{i}} w_{i}
$$

Alternatively, one might express the minimum rate of the bin as $\log |\mathcal{V}|-\bar{H}_{s}^{\mathcal{V}}(V \mid S)$, where, as before

$$
\bar{H}_{s}^{\mathcal{V}}(V \mid S)=\log |\mathcal{V}|-\bar{I}_{s}^{\mathcal{V}}(V ; S),
$$


is defined as the source coding group entropy of an Abelian group $\mathcal{V}$ and $\bar{H}_{s}^{\mathcal{V}}(V)=\bar{H}_{s}^{\mathcal{V}}(V \mid 0)$. We note that definitions (44) and (45) defined for an arbitrary Abelian group reduces to that in (40) and (41) for a $\mathbb{Z}_{p^{r}}$-group. This enables us to characterize an achievable rate region for MAC-DSTx based on Abelian group codes using $\mathcal{R}_{s g}(\tau)$.

Definition 13: Let $\mathbb{D}_{s g}(\boldsymbol{\tau}) \subseteq \mathbb{D}(\boldsymbol{\tau})$ be the collection of distributions $p_{\boldsymbol{U} \boldsymbol{V} \boldsymbol{S} \boldsymbol{X} Y}$ on $(\mathcal{U} \times \mathcal{V})^{2} \times \mathcal{S} \times \mathcal{X} \times \mathcal{Y}$ where $\mathcal{U}$ is a finite set and $\mathcal{V}$ is an Abelian group. For $p_{\boldsymbol{U} \boldsymbol{V} \boldsymbol{S} \boldsymbol{X} Y} \in \mathbb{D}_{s g}(\boldsymbol{\tau})$, let $\mathcal{R}_{s g}\left(p_{\boldsymbol{U} \boldsymbol{V} \boldsymbol{S} \boldsymbol{X} Y}\right)$ be defined as the set in 42 and $\mathcal{R}_{s g}(\boldsymbol{\tau})$ as in 43 .

We conclude by stating that $\mathcal{R}_{s g}(\tau)$ is indeed achievable.

Theorem 7: $\mathcal{R}_{s g}(\tau) \subseteq \mathbb{C}(\tau)$.

Remark 5: The persistent reader will recognize that the achievable rate region based on group codes hinges on the characterization of the minimum rate of a bin that is closed under group addition with respect to a test channel $p_{V \mid S}$. For the general Abelian group we stated this to be (44). Recent pursuit has resulted in further reduction of this quantity and is available in [40].

Remark 6: The results in this section point to a rich theory of strategies for multi-terminal communication systems based on structured code ensembles. Gains crucially rely on the compressive nature of the bivariate function and the ability to build efficient codes with rich algebraic structure. It is therefore no surprise that all of earlier findings were based on exploiting modulo-2 sum - the simplest compressive function with binary arguments - using linear codes - an ensemble that has been studied at length from different perspectives.

\section{CONCLUding Remarks}

We provided a single letter characterization of a new achievable rate region for the general MACDSTx. The reader will recognize that our findings are aimed at developing a new framework for obtaining achievable rate region for multi-terminal communication problems based on algebraic tools. We proposed achievable rate regions for an arbitrary MAC-DSTx based on two algebraic structures - fields and Abelian groups. It should now be clear to a persistent reader that a general rate region will involve a closure over all algebraic structures of which fields and Abelian groups are just two of them. Furthermore, this rate region will also incorporate the unstructured coding as indicated in section VII Indeed, a description of this will be involved, and is justified by the presence of additional degrees of freedom in the multi-terminal communication settings. 


\section{APPENDIX A}

\section{AN UPPER BOUND ON $P\left(\epsilon_{1}^{c} \cap \epsilon_{2}\right)$}

Through out this appendix $\pi$ denotes $\pi\left(\min \left\{(|\mathcal{X}| \cdot|\mathcal{S}|)^{2},(|\mathcal{X}|+|\mathcal{S}|+|\mathcal{Y}|-2) \cdot|\mathcal{X}| \cdot|\mathcal{S}|\right\}\right)$ and $\mathcal{V}$ : $=\mathcal{F}_{\pi}$. We begin with a simple lemma. The following lemma holds for any $\mathcal{F}_{q}$ and we state it in this generality.

Lemma 6: Let $\mathcal{F}_{q}$ be the finite field of cardinality $q$. If generator matrices $G_{I} \in \mathcal{F}_{q}^{k \times n}, G_{O / I} \in$ $\mathcal{F}_{q}^{l \times n}$ and bias vector $B^{n} \in \mathcal{F}_{q}^{n}$ of the random nested coset code $\left(n, k, l, G_{I}, G_{O / I}, B^{n}\right)$ are mutually independent and uniformly distributed on their respective range spaces, then codewords $V^{n}\left(a^{k}, m^{l}\right)$ : $=a^{k} G_{I} \oplus m^{l} G_{O / I} \oplus B^{n}$ are (i) uniformly distributed, and (ii) pairwise independent.

The proof follows form a simple counting argument and is omitted for the sake of brevity. The proof for the case $q=2$ is provided in [6, Theorem 6.2.1] and the same argument holds for any field $\mathcal{F}_{q}$.

We derive an upper bound on $P\left(\epsilon_{1}^{c} \cap \epsilon_{2}\right)$ using a second moment method similar to that employed in [41].

$$
\begin{aligned}
P\left(\epsilon_{1}^{c} \cap \epsilon_{2}\right) & =\sum_{s^{n} \in T_{\frac{\delta}{4}}\left(p_{S}\right)} \sum_{m^{l} \in \mathcal{V}^{l}} P\left(\begin{array}{c}
S^{n}=s^{n}, M^{l}=m^{l} \\
\phi_{\frac{\delta}{2}}\left(s^{n}, m^{l}\right)=0
\end{array}\right)=\sum_{s^{n} \in T_{\frac{\delta}{4}}(S)} \sum_{m^{l} \in \mathcal{V}^{l}} P\left(\begin{array}{l}
S^{n}=s^{n} \\
M^{l}=m^{i}
\end{array}\right) P\left(\phi_{\frac{\delta}{2}}\left(s^{n}, m^{l}\right)=0\right)(46) \\
& \leq \sum_{s^{n} \in T_{\frac{\delta}{4}}(S)} \sum_{m^{l} \in \mathcal{V}^{l}} P\left(S^{n}=s^{n}, M^{l}=m^{l}\right) P\left(\left|\phi_{\frac{\delta}{2}}\left(s^{n}, m^{l}\right)-\mathbb{E} \phi_{\frac{\delta}{2}}\left(s^{n}, m^{l}\right)\right| \geq \mathbb{E} \phi_{\frac{\delta}{2}}\left(s^{n}, m^{l}\right)\right) \\
& \leq \sum_{s^{n} \in T_{\frac{\delta}{4}}(S)} \sum_{m^{l} \in \mathcal{V}^{l}} P\left(S^{n}=s^{n}, M^{l}=m^{l}\right) \frac{\operatorname{Var}\left\{\phi_{\frac{\delta}{2}}\left(s^{n}, m^{l}\right)\right\}}{\left\{\mathbb{E}\left\{\phi_{\frac{\delta}{2}}\left(s^{n}, m^{l}\right)\right\}\right\}^{2}}
\end{aligned}
$$

where (46) is true since $\phi_{\frac{\delta}{2}}\left(s^{n}, m^{l}\right)$ is a function of random objects $G_{I}, G_{O / I}$ and $B^{n}$ that are mutually independent of $S^{n}, M^{l}$, and 47 follows from Cheybyshev inequality.

We now evaluate first and second moments of $\phi_{\frac{\delta}{2}}\left(s^{n}, m^{l}\right)$. The expectation of $\phi_{\frac{\delta}{2}}\left(s^{n}, m^{l}\right)$ is

$$
\mathbb{E} \phi_{\frac{\delta}{2}}\left(s^{n}, m^{l}\right)=\sum_{v^{n} \in T_{\frac{\delta}{2}}^{n}\left(V \mid s^{n}\right)} \sum_{a^{k} \in \mathcal{V}^{k}} P\left(V^{n}\left(a^{k}, M^{l}\right)=v^{n}\right)=\frac{\left|T_{\frac{\delta}{2}}^{n}\left(V \mid s^{n}\right)\right|}{\pi^{n-k}},
$$

where the last equality follows from Lemma 6(i). The second moment is

$$
\begin{aligned}
& \mathbb{E} \phi_{\frac{\delta}{2}}^{2}\left(s^{n}, m^{l}\right)=\sum_{v^{n}, \tilde{v}^{n} \in T_{\frac{\delta}{2}}^{n}\left(V \mid s^{n}\right)} \sum_{a^{k}, \tilde{a}^{k} \in \mathcal{V}^{k}} P\left(V^{n}\left(a^{k}, M^{l}\right)=v^{n}, V^{n}\left(\tilde{a}^{k}, M^{l}\right)=\tilde{v}^{n}\right) \\
& =\sum_{\substack{v^{n} \in \\
T_{\frac{\delta}{2}}^{n}\left(V \mid s^{n}\right)}} \sum_{a^{k} \in \mathcal{V}^{k}} P\left(V^{n}\left(a^{k}, M^{l}\right)=v^{n}\right)+\sum_{\substack{v^{n}, \tilde{v}^{n} \in \\
T_{\frac{\delta}{2}}^{n}\left(V \mid s^{n}\right)}} \sum_{\substack{a^{k}, \tilde{\mathcal{V}}^{k} \in a^{k} \neq \tilde{a}^{k} \\
a^{k}}} P\left(V^{n}\left(a^{k}, M^{l}\right)=v^{n}, V^{n}\left(\tilde{a}^{k}, M^{l}\right)=\tilde{v}^{n}\right) \\
& =\frac{\pi^{k}\left|T_{\frac{\delta}{4}}^{n}\left(V \mid s^{n}\right)\right|}{\pi^{n}}+\frac{\left|T_{\frac{\delta}{2}}^{n}\left(V \mid s^{n}\right)\right|^{2} \pi^{k}\left(\pi^{k}-1\right)}{\pi^{2 n}},
\end{aligned}
$$


where second term in (48) follows from Lemma 6(ii). Substituting for first and second moments of $\phi_{\frac{\delta}{2}}\left(s^{n}, m^{l}\right)$, we have

$\operatorname{Var}\left\{\phi_{\frac{\delta}{2}}\left(s^{n}, m^{l}\right)\right\}=\frac{\pi^{k}\left|T_{\frac{\delta}{2}}^{n}\left(V \mid s^{n}\right)\right|}{\pi^{n}}\left(1-\frac{\left|T_{\frac{\delta}{2}}^{n}\left(V \mid s^{n}\right)\right|}{\pi^{n}}\right)$, thus $\frac{\operatorname{Var}\left\{\phi_{\frac{\delta}{2}}\left(s^{n}, m^{l}\right)\right\}}{\mathbb{E}\left\{\phi_{\frac{\delta}{2}}\left(s^{n}, m^{l}\right)\right\}^{2}} \leq \frac{\pi^{n-k}}{\left|T_{\frac{\delta}{2}}^{n}\left(V \mid s^{n}\right)\right|}$.

For $s^{n} \in T_{\frac{\delta}{4}}(S)$ lemma 5 , guarantees existence of $N_{3}(\eta) \in \mathbb{N}$, such that for all $n \geq N_{3}(\eta),\left|T_{\frac{\delta}{2}}\left(V \mid s^{n}\right)\right| \geq$ $\exp \left\{n\left(H(V \mid S)-\frac{3 \delta}{4}\right)\right\}$. Substituting this lower bound in 49 , we note,

$$
\frac{\operatorname{Var}\left\{\phi_{\frac{\delta}{2}}\left(s^{n}, m^{l}\right)\right\}}{\mathbb{E}\left\{\phi_{\frac{\delta}{2}}\left(s^{n}, m^{l}\right)\right\}^{2}} \leq \frac{\pi^{n-k}}{\left|T_{\frac{\delta}{2}}^{n}\left(V \mid s^{n}\right)\right|} \leq \exp \left\{-n \log \pi\left(\frac{k}{n}-\left(1-\frac{H(V \mid S)}{\log \pi}+\frac{3 \delta}{4 \log \pi}\right)\right)\right\}
$$

Substituting (50) in (47), we obtain

$$
P\left(\epsilon_{1}^{c} \cap \epsilon_{2}\right) \leq \exp \left\{-n \log \pi\left(\frac{k}{n}-\left(1-\frac{H(V \mid S)}{\log \pi}+\frac{3 \delta}{8 \log \pi}\right)\right)\right\} .
$$

From (3), we have

$$
\frac{k}{n}-\left(1-\frac{H(V \mid S)}{\log \pi}+\frac{3 \delta}{8 \log \pi}\right) \geq \frac{\frac{\eta}{8}-\frac{3 \delta}{8}}{\log \pi} \geq \frac{\eta}{16 \log \pi}
$$

where the last inequality follows from choice of $\delta$. Combining 51] and 51), we have $P\left(\epsilon_{1}^{c} \cap \epsilon_{2}\right) \leq$ $\exp \left\{-\frac{3 n \delta}{8 \log \pi}\right\} \leq \frac{\eta}{16}$ for all $n \geq N_{4}(\eta)$.

By choosing $\delta>0$ sufficiently small, $\frac{k}{n}$ can be made arbitrarily close to $1-\frac{H(V \mid S)}{\log \pi}$ and probability of encoding error can be made arbitrarily small by choosing a sufficiently large block length. The above findings are summarized in the following lemma.

Lemma 7: Let $\mathcal{S}$ be a finite set, $\mathcal{V}=\mathcal{F}_{q}$ a finite field and $p_{S V}$, a pmf on $\mathcal{S} \times \mathcal{V}$. Consider a random nested coset code $\left(n, k, l, G_{I}, G_{O / I}, B^{n}\right)$ denoted $\Lambda_{O} / \Lambda_{I}$, with bias vector $B^{n} \in \mathcal{V}^{n}$, generator matrices $G_{I} \in \mathcal{V}^{k \times n}$ and $G_{O / I} \in \mathcal{V}^{l \times n}$ mutually independent and uniformly distributed on their respective range spaces. Let $V^{n}\left(a^{k}, m^{l}\right):=a^{k} G_{I} \oplus m^{l} G_{O / I} \oplus B^{n}$ denote generic codeword in $\Lambda_{O} / \Lambda_{I}$. For $s^{n} \in \mathcal{S}^{n}$, $m^{l} \in \mathcal{V}^{l}$ and $\delta>0$, let $\phi_{\delta}\left(s^{n}, m^{l}\right):=\sum_{a^{k} \in \mathcal{V}^{k}} 1_{\left\{\left(s^{n}, V^{n}\left(a^{k}, m^{l}\right)\right) \in T_{\delta}(S, V)\right\}}$. The following are true.

1) The codewords $V^{n}\left(a^{k}, m^{l}\right): a^{k} \in \mathcal{V}^{k}$ are uniformly distributed and pairwise independent.

2) For any $\delta>0, s^{n} \in T_{\frac{\delta}{2}}(S), m^{l} \in \mathcal{V}^{l}$, there exists $N(\delta) \in \mathbb{N}$ such that for all $n \geq N(\delta)$,

$$
P\left(\phi_{\delta}\left(s^{n}, m^{l}\right)=0\right) \leq \exp \left\{-n \log q\left(\frac{k}{n}-\left(1-\frac{H(V \mid S)}{\log q}-\frac{3 \delta}{2 \log q}\right)\right)\right\} .
$$

3) If $\left(S^{n}, M^{l}\right) \in \mathcal{S}^{n} \times \mathcal{V}^{l}$ are independent of $\left(G_{I}, G_{O / I}, B^{n}\right)$, then for all $n \geq N(\delta)$,

$$
P\left(S^{n} \in T_{\frac{\delta}{2}}(S), \phi_{\delta}\left(S^{n}, M^{l}\right)=0\right) \leq \exp \left\{-n \log q\left(\frac{k}{n}-\left(1-\frac{H(V \mid S)}{\log q}-\frac{3 \delta}{2 \log q}\right)\right)\right\} .
$$




\section{APPENDIX B}

\section{AN UPPER BOUND ON $P\left(\left(\epsilon_{1} \cup \epsilon_{2} \cup \epsilon_{3}\right)^{c} \cap \epsilon_{4}\right)$}

As is typical, our achievability proof hinges on independence of transmitted codeword (and hence received vector) and the contending codewords that are not transmitted. Towards this end, we begin with the following.

Lemma 8: Let $\mathcal{V}$ be the finite field of cardinality $q$. If generator matrices $G_{I} \in \mathcal{F}_{q}^{k \times n}, G_{O / I} \in \mathcal{V}^{l \times n}$ and bias vector $B^{n} \in \mathcal{F}_{q}^{n}$ of the random $\left(n, k, l, G_{I}, G_{O / I}, B^{n}\right)$ nested coset code are mutually independent and uniformly distributed on their respective range spaces, then any coset is independent of any codeword in a different coset., i.e., the collection of codewords $\left(V^{n}\left(a^{k}, m^{l}\right): a^{k} \in \mathcal{F}_{q}^{k}\right)$ and $V^{n}\left(\hat{a}^{k}, \hat{m}^{l}\right)$ are independent if $m^{l} \neq \hat{m}^{l}$.

Proof: Let $v_{a^{k}}^{n} \in \mathcal{F}_{q}^{n}$ for each $a^{k} \in \mathcal{F}_{q}^{k}$, and $\hat{v}^{n} \in \mathcal{F}_{q}^{n}$. We need to prove $P\left(V^{n}\left(a^{k}, m^{l}\right)=v_{a^{k}}^{n}: a^{k} \in \mathcal{F}_{q}^{k}, V^{n}\left(\hat{a}^{k}, m^{l}\right)=\hat{v}^{n}\right)=P\left(V^{n}\left(a^{k}, m^{l}\right)=v_{a^{k}}^{n}: a^{k} \in \mathcal{F}_{q}^{k}\right) P\left(V^{n}\left(\hat{a}^{k}, m^{l}\right)=\hat{v}^{n}\right)$. If $\left(v_{a^{k}+\hat{a}^{k}}^{n}-v_{0^{k}}^{n}\right) \neq\left(v_{a^{k}}^{n}-v_{0^{k}}^{n}\right)+\left(v_{\hat{a}^{k}}^{n}-v_{0^{k}}^{n}\right)$ for some pair $a^{k}, \hat{a}^{k} \in \mathcal{F}_{q}^{k}$, the LHS and first term of RHS are zero and equality holds. Else,

$$
\begin{aligned}
P & \left(V^{n}\left(a^{k}, m^{l}\right)=v_{a^{k}}^{n}: a^{k} \in \mathcal{F}_{q}^{k}, V^{n}\left(\hat{a}^{k}, m^{l}\right)=\hat{v}^{n}\right) \\
& =P\left(a^{k} G_{I}=v_{a^{k}}^{n}-v_{0^{k}}^{n}: a^{k} \in \mathcal{F}_{q}^{k}, V^{n}\left(0^{k}, m^{l}\right)=v_{0^{k}}^{n}, V^{n}\left(0^{k}, \hat{m}^{l}\right)=\hat{v}^{n}-v_{\hat{a}^{k}}^{n}\right) \\
& =P\left(a^{k} G_{I}=v_{a^{k}}^{n}-v_{0^{k}}^{n}: a^{k} \in \mathcal{F}_{q}^{k}\right) P\left(V^{n}\left(0^{k}, m^{l}\right)=v_{0^{k}}^{n}, V^{n}\left(0^{k}, \hat{m}^{l}\right)=\hat{v}^{n}-v_{\hat{a}^{k}}^{n}\right) \\
& =P\left(a^{k} G_{I}=v_{a^{k}}^{n}-v_{0^{k}}^{n}: a^{k} \in \mathcal{F}_{q}^{k}\right) P\left(V^{n}\left(0^{k}, m^{l}\right)=v_{0^{k}}^{n}\right) P\left(V^{n}\left(0^{k}, \hat{m}^{l}\right)=\hat{v}^{n}-v_{\hat{a}^{k}}^{n}\right) \\
& =P\left(a^{k} G_{I}=v_{a^{k}}^{n}-v_{0^{k}}^{n}: a^{k} \in \mathcal{F}_{q}^{k}, V^{n}\left(0^{k}, m^{l}\right)=v_{0^{k}}^{n}\right) P\left(\hat{m}^{l} G_{O / I}+B^{n}=\hat{v}^{n}-v_{\hat{a}^{k}}^{n}\right) \\
& =P\left(V^{n}\left(a^{k}, m^{l}\right)=v_{a^{k}}^{n}: a^{k} \in \mathcal{F}_{q}^{k}\right) P\left(V^{n}\left(\hat{a}^{k}, m^{l}\right)=\hat{v}^{n},\right)
\end{aligned}
$$

where (52) and (54) follow from independence of $G_{O / I}, B^{n}$ and $G_{I} \sqrt{53}$ follows from Lemma 6(ii), and the last equality follows from invariance of the pmf of $V^{n}\left(a^{k}, m^{l}\right)$ with respect to $a^{k}$ and $m^{l}$. We emphasize the consequence of Lemma 8 in the following remark.

Remark 7: If transmitted message $M^{l} \neq \hat{m}^{l}$, then $Y^{n}$ is independent of $V^{n}\left(\hat{a}^{k}, \hat{m}^{l}\right)$. Indeed

$$
\begin{aligned}
& P\left(V^{n}\left(\hat{a}^{k}, \hat{m}^{l}\right)=\hat{v}^{n}, Y^{n}=y^{n}\right)=\sum_{\left(v_{a^{n}}^{n} \in \mathcal{V}^{n}: a^{k} \in \mathcal{V}^{k}\right)} \sum_{x^{n} \in \mathcal{X}^{n}} P\left(\begin{array}{c}
C\left(M^{l}\right)=\left(v_{a^{k}}^{n} \in \mathcal{V}^{n}: a^{k} \in \mathcal{V}^{k}\right), \\
V^{n}\left(\hat{a}^{k}, \hat{m}^{l}\right)=\hat{v}^{n}, E\left(S^{n}, M^{l}\right)=x^{n}, Y^{n}=y^{n}
\end{array}\right) \\
& =\sum_{\left(v_{a^{n}}^{n} \in \mathcal{V}^{n}: a^{k} \in \mathcal{V}^{k}\right) x^{n} \in \mathcal{X}^{n}} P\left(\begin{array}{l}
C\left(M^{l}\right)=\left(v_{a^{k}}^{n} \in \mathcal{V}^{n}: a^{k} \in \mathcal{V}^{k}\right), \\
E\left(S^{n}, M^{l}\right)=x^{n}, Y^{n}=y^{n}
\end{array}\right) P\left(V^{n}\left(\hat{a}^{k}, \hat{m}^{l}\right)=\hat{v}^{n}\right) \\
& =P\left(V^{n}\left(\hat{a}^{k}, \hat{m}^{l}\right)=\hat{v}^{n}\right) P\left(Y^{n}=y^{n}\right)=\frac{P\left(Y^{n}=y^{n}\right)}{q^{n}} .
\end{aligned}
$$


We have used (1) independence of $V^{n}\left(\hat{a}^{k}, \hat{m}^{l}\right)$ and $C\left(M^{l}\right)$ (lemma 8), (2) $E\left(S^{n}, M^{l}\right)$ being a function of $C\left(M^{l}\right)$ and $S^{n}$ is conditionally independent of $V^{n}\left(\hat{a}^{k}, \hat{m}^{l}\right)$ given $C\left(M^{l}\right)$, and (3) $Y^{n}$ is conditionally independent of $V^{n}\left(\hat{a}^{k}, \hat{m}^{l}\right)$ given $E\left(S^{n}, M^{l}\right)$ in arriving at 55), and lemma 6(i) in arriving at the last equality in (56).

We now provide an upper bound on $P\left(\left(\epsilon_{1} \cup \epsilon_{2} \cup \epsilon_{3}\right)^{c} \cap \epsilon_{4}\right)$. Observe that

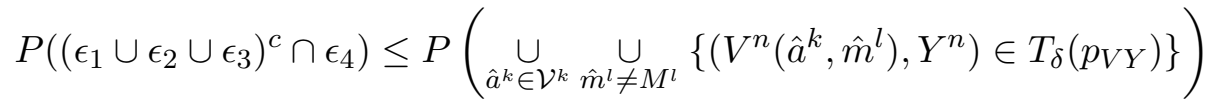

$$
\begin{aligned}
& \leq \sum_{\substack{\hat{h}^{l} \in \mathcal{V}^{l} \\
\hat{m}^{l} \neq M^{l}}} \sum_{\hat{a}^{k} \in \mathcal{V}^{k}} \sum_{\substack{y^{n} \\
\in T_{\frac{\delta}{2}}}} \sum_{\substack{v_{\delta} \\
T^{n} \in \\
\left(V \mid y^{n}\right)}} P\left(V^{n}\left(\hat{a}^{k}, \hat{m}^{l}\right)=v^{n}, Y^{n}=y^{n}\right) \\
& =\sum_{\substack{\hat{m}^{l} \in \mathcal{V}^{l} \\
\hat{m}^{l} \neq M^{l}}} \sum_{\hat{a}^{k} \in \mathcal{V}^{k}} \sum_{\substack{y^{n} \\
\in T_{\frac{\delta}{2}}}} \sum_{\substack{v^{n} \in \\
T_{\delta}\left(V \mid y^{n}\right)}} P\left(V^{n}\left(\hat{a}^{k}, \hat{m}^{l}\right)=v^{n}\right) P\left(Y^{n}=y^{n}\right)=\sum_{\substack{\hat{m}^{l} \in \mathcal{V}^{l} \\
\hat{m}^{l} \neq M^{l}}} \sum_{\hat{a}^{k} \in \mathcal{V}^{k}} \sum_{\substack{y^{n} \\
\in T_{\frac{\delta}{2}}}} \sum_{\substack{v_{\delta}^{n} \in\left(V \mid y^{n}\right) \\
T^{n}}} \frac{P\left(Y^{n}=y^{n}\right)}{\pi^{n}}(57) \\
& \leq \sum_{y^{n} \in T_{\frac{\delta}{2}}} \frac{\pi^{k+l}\left|T_{\delta}\left(p_{V \mid Y} \mid y^{n}\right)\right| P\left(Y^{n}=y^{n}\right)}{\pi^{n}},
\end{aligned}
$$

where, the two equalities in 57 follow from (56). Lemma 5 guarantees existence of $N_{5}(\eta) \in \mathbb{N}$ such that for all $n \geq N_{5}(\eta)$ and $y^{n} \in T_{\frac{\delta}{2}}\left(p_{Y}\right),\left|T_{\delta}\left(V \mid y^{n}\right)\right| \leq \exp \left\{n\left(H(V \mid Y)+\frac{3 \delta}{2}\right)\right\}$. Substituting this upper bound in (58), we conclude

$$
P\left(\left(\epsilon_{1} \cup \epsilon_{2} \cup \epsilon_{3}\right)^{c} \cap \epsilon_{4}\right) \leq \exp \left\{-n \log \pi\left(1-\frac{H(V \mid Y)}{\log \pi}-\frac{3 \delta}{2 \log \pi}-\frac{k+l}{n}\right)\right\}
$$

for all $n \geq N_{5}(\eta)$.

\section{APPENDIX C}

\section{AN UPPER BOUND ON $P\left(\epsilon_{5}\right)$}

In this appendix, we derive an upper bound on $P\left(\epsilon_{5}\right)$. As is typical in proofs of channel coding theorems, this step involves establishing statistical independence of cosets $C_{j}\left(M_{j}^{l_{j}}\right): j=1,2$ corresponding to the message pair and any codeword $V^{n}\left(\hat{a}^{k}, \hat{m}^{l}\right)$ in a competing coset. We establish this in lemma 10. We begin with the necessary spadework. Throughout this appendix, we employ the notation introduced in proof of theorem 4

Lemma 9: If $m^{l} \neq \hat{m}^{l}$, then for any triple $\nu_{1}, \nu_{2}, \hat{\nu} \in \mathcal{V}^{n}$,

$$
P\left(\begin{array}{c}
V_{j}^{n}\left(0^{k_{j}}, m_{j}^{l_{j}}\right)=\nu_{j}^{n}: j=1,2, \\
V^{n}\left(0^{k}, \hat{m}^{l}\right)=\hat{\nu}^{n}
\end{array}\right)=P\left(V_{j}^{n}\left(0^{k_{j}}, m_{j}^{l_{j}}\right)=\nu_{j}^{n}: j=1,2\right) P\left(V^{n}\left(0^{k}, \hat{m}^{l}\right)=\hat{\nu}^{n}\right)
$$


Proof: By definition of $V_{j}\left(0_{k_{j}}, m_{j}^{l_{j}}\right): j=1,2$ and $V\left(0^{k}, m^{l}\right)$,

$$
\begin{aligned}
& P\left(\begin{array}{c}
V_{j}^{n}\left(0^{k_{j}}, m_{j}^{l_{j}}\right)=\nu_{j}^{n}: j=1,2, \\
V^{n}\left(0^{k}, \hat{m}^{l}\right)=\hat{\nu}^{n}
\end{array}\right)=P\left(\begin{array}{cc}
{\left[\begin{array}{cc}
m_{1}^{l_{1}} & 0^{l_{2}}
\end{array}\right] G_{O / I} \oplus B_{1}^{n}=\nu_{1}^{n},\left[\begin{array}{ll}
0_{1}^{l_{1}} & m_{2}^{l_{2}}
\end{array}\right] G_{O / I} \oplus B_{2}^{n}=\nu_{2}^{n}} \\
{\left[\begin{array}{cc}
\hat{m}_{1}^{l_{1}} & \hat{m}_{2}^{l_{2}}
\end{array}\right] G_{O / I} \oplus B_{1}^{n} \oplus B_{2}^{n}=\hat{\nu}^{n}}
\end{array}\right)
\end{aligned}
$$

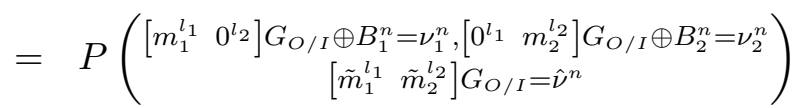

where $\tilde{m}_{j}^{l_{j}}=\hat{m}_{j}^{l_{j}}-m_{j}^{l_{j}}$. We now prove, using a counting argument similar to that employed in proof of lemma 6 , the term on right hand side of $\sqrt[60]{ }$ is $\frac{1}{\pi^{3 n}}$. Since $\hat{m}^{l} \neq m^{l}$, there exists $t \in[l]$ such that $\hat{m}_{t} \neq m_{t}$. Given any $(l-1)$ vectors $g_{O / I, j} \in \mathcal{V}^{n}: j \in[l] \backslash\{t\}$, there exists a unique triple of vectors $\left(g_{O / I, t}, b_{1}^{n}, b_{2}^{n}\right) \in \mathcal{V}^{n} \times \mathcal{V}^{n} \times \mathcal{V}^{n}$ such that $\left[\begin{array}{ll}m_{1}^{l_{1}} & 0^{l_{2}}\end{array}\right] g_{O / I} \oplus b_{1}^{n}=\nu_{1}^{n},\left[\begin{array}{ll}0^{l_{1}} & m_{2}^{l_{2}}\end{array}\right] g_{O / I} \oplus b_{2}^{n}=\nu_{2}^{n}$ and $\left[\begin{array}{cc}\tilde{m}_{1}^{l_{1}} & \tilde{m}_{2}^{l_{2}}\end{array}\right] g_{O / I}=\hat{\nu}^{n}$, where row $j$ of $g_{O / I}$ is $g_{O / I, j}$. Hence

$$
\mid\left\{\left(g_{O / I}, b_{1}^{n}, b_{2}^{n}\right) \in \mathcal{V}^{k \times n} \times \mathcal{V}^{n} \times \mathcal{V}^{n}:\left[\begin{array}{cc}
m_{1}^{l_{1}} & \left.0^{l_{2}}\right] g_{O / I} \oplus B_{1}^{n}=\nu_{1},\left[\begin{array}{ll}
0^{l_{1}} & m_{2}^{l_{2}}
\end{array}\right] g_{O / I} \oplus B_{2}^{n}=\nu_{2} \\
{\left[\begin{array}{cc}
\tilde{m}_{1}^{l_{1}} & \tilde{m}_{2}^{l_{2}}
\end{array}\right] g_{O / I}=\hat{\nu}^{n}}
\end{array}\right\} \mid=\pi^{(l-1) n} .\right.
$$

The mutual independence and uniform distribution of $G_{O / I}, B_{1}, B_{2}^{n}$ implies the term on RHS of (60) is indeed $\frac{1}{\pi^{3 n}}$. It remains to prove

$$
P\left(V_{j}^{n}\left(0^{k_{j}}, m_{j}^{l_{j}}\right)=\nu_{j}^{n}: j=1,2\right) P\left(V^{n}\left(0^{k}, \hat{m}^{l}\right)=\hat{\nu}^{n}\right)=\frac{1}{\pi^{3 n}} .
$$

It follows from lemma 6 that $P\left(V^{n}\left(0^{k}, \hat{m}^{l}\right)=\hat{\nu}^{n}\right)=\frac{1}{\pi^{n}}$. Using the definition of $V^{n}\left(0^{k}, \hat{m}^{l}\right)$, we only need to prove

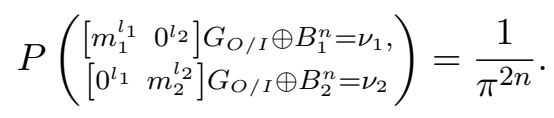

This follows again from a counting argument. For every matrix $g_{O / I} \in \mathcal{V}^{l \times n}$, there exists a unique pair of vectors $b_{1}^{n}, b_{2}^{n} \in \mathcal{V}^{n}$ such that $\left[\begin{array}{ll}m_{1}^{l_{1}} & 0^{l_{2}}\end{array}\right] G_{O / I} \oplus B_{1}^{n}=\nu_{1}$, and $\left[\begin{array}{ll}0^{l_{1}} & m_{2}^{l_{2}}\end{array}\right] G_{O / I} \oplus B_{2}^{n}=\nu_{2}$ thus yielding

$$
\mid\left\{\left(g_{O / I}, b_{1}^{n}, b_{2}^{n}\right) \in \mathcal{V}^{k \times n} \times \mathcal{V}^{n} \times \mathcal{V}^{n}:\left[\begin{array}{cc}
{\left[\begin{array}{cc}
m_{1}^{l_{1}} & 0^{l_{2}}
\end{array}\right] G_{O / I} \oplus B_{1}^{n}=\nu_{1}} \\
{\left[\begin{array}{ll}
0^{l_{1}} & m_{2}^{l_{2}}
\end{array}\right] G_{O / I} \oplus B_{2}^{n}=\nu_{2}}
\end{array}\right\} \mid=\pi^{l n},\right.
$$

and the proof is completed using the mutual independence and uniform distribution of $G_{O / I}, B_{1}^{n}, B_{2}^{n}$.

Lemma 10: For any $\hat{m}^{l} \neq m^{l}$, and any $\hat{a}^{k} \in \mathcal{V}^{k}$, the pair of cosets $C_{j}\left(m_{j}^{l_{j}}\right): j=1,2$ is statistically independent of $V^{n}\left(\hat{a}^{k}, \hat{m}^{l}\right)$.

Proof: For $j=1,2$, let $\nu_{j}^{n}\left(a_{j}^{k_{j}}\right) \in \mathcal{V}^{n}$ for each $a_{j}^{k_{j}} \in \mathcal{V}^{k_{j}}$, and $\hat{\nu}^{n} \in \mathcal{V}^{n}$. We need to prove $P\left(\begin{array}{l}C_{1}^{n}\left(m_{1}^{l_{1}}\right)=\left(\nu_{1}\left(a_{1}^{k_{1}}\right): a_{1}^{k_{1}} \in \mathcal{V}^{k_{1}}\right) \\ C_{2}^{n}\left(m_{2}^{l_{2}}\right)=\left(\nu_{2}\left(a_{2}^{k_{2}}\right): a_{2}^{k_{2}} \in \mathcal{V}^{k_{2}}\right) \\ V^{n}\left(\hat{a}^{k}, \hat{m}^{l}\right)=\hat{\nu}^{n}\end{array}\right)=P\left(\begin{array}{l}C_{1}^{n}\left(m_{1}^{l_{1}}\right)=\left(\nu_{1}\left(a_{1}^{k_{1}}\right): a_{1}^{k_{1}} \in \mathcal{V}^{k_{1}}\right) \\ C_{2}^{n}\left(m_{2}^{l_{2}}\right)=\left(\nu_{2}\left(a_{2}^{k_{2}}\right): a_{2}^{k_{2}} \in \mathcal{V}^{k_{2}}\right)\end{array}\right) P\left(\begin{array}{c}V^{n}\left(\hat{a}^{k}, \hat{m}^{l}\right) \\ =\hat{\nu}^{n}\end{array}\right)$ for every choice of $\nu_{j}\left(a_{j}^{k_{j}}\right) \in \mathcal{V}^{n}: a_{j}^{k_{j}} \in \mathcal{V}^{k_{j}}, j=1,2$ and $\hat{\nu}^{n} \in \mathcal{V}^{n}$. 
If (i) for some $j=1$ or $j=2,\left(\nu_{j}\left(a_{j}^{k_{j}} \oplus \tilde{a}_{j}^{k_{j}}\right)-\nu_{j}\left(0^{k_{j}}\right)\right) \neq\left(\nu_{j}\left(a_{j}^{k_{j}}\right)-\nu_{j}\left(0^{k_{j}}\right)\right) \oplus\left(\nu_{j}\left(\tilde{a}_{j}^{k_{j}}\right)-\nu_{j}\left(0^{k_{j}}\right)\right)$ for any pair $a_{j}^{k_{j}}, \tilde{a}_{j}^{k_{j}} \in \mathcal{V}^{k_{j}}$, or (ii) $\nu_{1}\left(a^{k_{1}}\right)-v_{1}\left(0^{k_{1}}\right) \neq \nu_{2}\left(a_{1}^{k_{1}} 0^{k_{+}}\right)-v_{2}\left(0^{k_{2}}\right)$ for some $a_{1}^{k_{1}} \in \mathcal{V}^{k_{1}}$, then LHS and first term of RHS are zero and equality holds. Otherwise,

$$
\begin{aligned}
& P\left(C_{j}^{n}\left(m_{j}^{l_{j}}\right)=\left(\nu_{j}\left(a_{j}^{k_{j}}\right): a_{j}^{k_{j}} \in \mathcal{V}^{k_{j}}\right): j=1,2, V^{n}\left(\hat{a}^{k}, \hat{m}^{l}\right)=\hat{\nu}^{n}\right) \\
& =P\left(\begin{array}{c}
a_{2}^{k_{2}} G_{I_{2}}=\nu_{2}\left(a^{k_{2}}\right)-\nu_{2}\left(0^{k_{2}}\right): a_{2}^{k_{2}} \in \mathcal{V}^{k_{2}}, V_{j}^{n}\left(0^{k_{j}}, m_{j}^{l_{j}}\right)=\nu_{j}\left(0^{k_{j}}\right): j=1,2, \\
V^{n}\left(0^{k}, \hat{m}^{l}\right)=\hat{\nu}^{n}-\left(\nu_{2}\left(\hat{a}^{k}\right)-\nu_{2}\left(0^{k_{2}}\right)\right)
\end{array}\right) \\
& =P\left(\begin{array}{c}
a_{2}^{k_{2}} G_{I_{2}}=\nu_{2}\left(a^{k_{2}}\right)- \\
\nu_{2}\left(0^{k_{2}}\right): a_{2}^{k_{2}} \in \mathcal{V}^{k_{2}}
\end{array}\right) P\left(\begin{array}{c}
V_{j}^{n}\left(0^{k_{j}}, m_{j}^{l_{j}}\right)=\nu_{j}\left(0^{k_{j}}\right): j=1,2, \\
V^{n}\left(0^{k}, \hat{m}^{l}\right)=\hat{\nu}^{n}-\left(\nu_{2}\left(\hat{a}^{k}\right)-\nu_{2}\left(0^{k_{2}}\right)\right)
\end{array}\right) \\
& =P\left(\begin{array}{c}
a_{2}^{k_{2}} G_{I_{2}}=\nu_{2}\left(a^{k_{2}}\right)- \\
\nu_{2}\left(0^{k_{2}}\right): a_{2}^{k_{2}} \in \mathcal{V}^{k_{2}}
\end{array}\right) P\left(\begin{array}{cc}
{\left[m_{1}^{l_{1}}\right.} & \left.0^{l_{2}}\right] G_{O / I} \oplus B_{1}^{n}=\nu_{1}\left(0^{k_{1}}\right), \\
{\left[0^{l_{1}}\right.} & \left.m_{2}^{l_{2}}\right] G_{O / I} \oplus B_{2}^{n}=\nu_{2}\left(0^{k_{2}}\right)
\end{array}\right) P\left(V^{n}\left(\hat{a}^{k}, \hat{m}^{l}\right)=\hat{\nu}^{n}\right) \\
& =P\left(\begin{array}{c}
a_{2}^{k_{2}} G_{I_{2}}=\nu_{2}\left(a^{k_{2}}\right)-\nu_{2}\left(0^{k_{2}}\right): a_{2}^{k_{2}} \in \mathcal{V}^{k_{2}} \\
{\left[\begin{array}{cc}
m_{1}^{l_{1}} & 0^{l_{2}}
\end{array}\right] G_{O / I} \oplus B_{1}^{n}=\nu_{1}\left(0^{k_{1}}\right),\left[\begin{array}{ll}
0^{l_{1}} & m_{2}^{l_{2}}
\end{array}\right] G_{O / I} \oplus B_{2}^{n}=\nu_{2}\left(0^{k_{2}}\right)}
\end{array}\right) P\left(V^{n}\left(\hat{a}^{k}, \hat{m}^{l}\right)=\hat{\nu}^{n}\right) \\
& =P\left(C_{j}^{n}\left(m_{j}^{l_{j}}\right)=\left(\nu_{j}\left(a_{j}^{k_{j}}\right): a_{j}^{k_{j}} \in \mathcal{V}^{k_{j}}\right): j=1,2\right) P\left(V^{n}\left(\hat{a}^{k}, \hat{m}^{l}\right)=\hat{\nu}^{n}\right)
\end{aligned}
$$

where i) 64 and 66 follow from definition of cosets $C_{j}\left(m_{j}^{l_{j}}\right)$, (ii) 63 and 65 follow from independence of $G_{I_{2}}$ and the collection $\left(G_{O / I}, B_{1}^{n}, B_{2}^{n}\right)$ and (iii) 62 follows from lemma 9 .

We emphasize consequence of lemma 10 in the following remark.

Remark 8: If $m^{l} \neq \hat{m}^{l}$, then conditioned on event $\left\{M^{l}=m^{l}\right\}$, received vector $Y^{n}$ is statistically independent of $V^{n}\left(\hat{a}^{k}, \hat{m}^{l}\right)$ for any $\hat{a}^{k} \in \mathcal{V}^{k}$. We establish truth of this statement in the sequel. Let $\mathcal{C}_{j}$ denote the set of all ordered $\pi^{k_{j}}$-tuples of vectors in $\mathcal{V}^{n}$. Observe that

$$
\begin{aligned}
P & \left(\begin{array}{c}
M^{l}=m^{l}, Y^{n}=y^{n}, \\
V^{n}\left(\hat{a}^{k}, \hat{m}^{l}\right)=\hat{\nu}^{n}
\end{array}\right)=\sum_{C_{1} \in \mathcal{C}_{1}} \sum_{C_{2} \in \mathcal{C}_{2}} \sum_{s^{n} \in \mathcal{S}^{n}} P\left(\begin{array}{c}
M^{l}=m^{l}, C_{j}\left(m_{j}^{l_{j}}\right)=C_{j}: j=1,2, S^{n}=s^{n} \\
V^{n}\left(\hat{a}^{k}, \hat{m}^{l}\right)=\hat{v}^{n}, Y^{n}=y^{n}
\end{array}\right) \\
& =\sum_{C_{1} \in \mathcal{C}_{1}} \sum_{C_{2} \in \mathcal{C}_{2}} \sum_{s^{n} \in \mathcal{S}^{n}} P\left(\begin{array}{c}
\left.M^{l}=m^{l}\right) \\
S^{n}=s^{n}
\end{array}\right) P\left(\begin{array}{c}
C_{1}\left(m_{1}^{l_{1}}\right)=C_{1} \\
C_{2}\left(m_{2}^{l_{2}}\right)=C_{2}
\end{array}\right) P\left(V^{n}\left(\hat{a}^{k}, \hat{m}^{l}\right)=\hat{v}^{n}\right) P\left(Y^{n}=y^{n} \mid \begin{array}{c}
C_{j}\left(m_{j}^{l_{j}}\right)=C_{j}: j=1,2 \\
S^{n}=s^{n}, M^{l}=m^{l}
\end{array}\right)(67) \\
& =\sum_{C_{1} \in \mathcal{C}_{1}} \sum_{C_{2} \in \mathcal{C}_{2}} \sum_{s^{n} \in \mathcal{S}^{n}} P\left(\begin{array}{c}
M^{l}=m^{l}, Y^{n}=y^{n}, S^{n}=s^{n} \\
C_{j}\left(m_{j}^{l_{j}}\right)=C_{j}: j=1,2
\end{array}\right) P\left(V^{n}\left(\hat{a}^{k}, \hat{m}^{l}\right)=\hat{v}^{n}\right) \\
& =P\left(M^{l}=m^{l}, Y^{n}=y^{n}\right) P\left(V^{n}\left(\hat{a}^{k}, \hat{m}^{l}\right)=\hat{\nu}^{n}\right)
\end{aligned}
$$

where 67) follows from (i) independence of random objects that characterize codebook and $\left(S^{n}, M^{l}\right)$, (ii) lemma 10 and (iii) statistical independence of the inputs $X_{j}\left(M_{j}^{l_{j}}, S_{j}^{n}\right): j=1,2$ to the channel and the codeword $V^{n}\left(\hat{a}^{k}, \hat{m}^{l}\right)$ conditioned on the specific realization of cosets $\left(C_{j}\left(M_{j}^{l_{j}}\right): j=1,2\right)$ and the event $\left\{M^{l}=m^{l}\right\}$. Moreover, since $P\left(V^{n}\left(\hat{a}^{k}, \hat{m}^{l}\right)=\hat{\nu}^{n}\right)=\frac{1}{\pi^{n}}$, we have $P\left(M^{l}=m^{l}, Y^{n}=\right.$ $\left.y^{n}, V^{n}\left(\hat{a}^{k}, \hat{m}^{l}\right)=\hat{\nu}^{n}\right)=\frac{1}{\pi^{n}} P\left(M^{l}=m^{l}, Y^{n}=y^{n}\right)$. 
We are now equipped to derive an upper bound on $P\left(\epsilon_{5}\right)$. Observe that

$$
\begin{aligned}
& P\left(\epsilon_{5}\right) \leq P\left(\bigcup_{\hat{a}^{k} \in \mathcal{V}^{k}} \bigcup_{\substack{m^{l}, \hat{m}^{l} \\
m^{l} \neq \hat{m}^{l}}}\left\{\begin{array}{c}
\left(V^{n}\left(\hat{a}^{k}, \hat{m}^{l}\right), Y^{n}\right) \in T_{\eta_{5}(\eta)}\left(p_{V_{1} \oplus V_{2}, Y}\right) \\
M^{l}=m^{l}
\end{array}\right\}\right) \\
& \leq \sum_{\hat{a}^{k} \in \mathcal{V}^{k}} \sum_{\substack{m^{l}, \hat{m}^{l} \\
m^{l} \neq \hat{m}^{l} \in T_{\eta_{5}(\eta)}(Y)}} \sum_{\substack{y^{n} \\
T_{\eta_{5}(\eta)}\left(V_{1} \in V_{2} \mid y^{n}\right)}} P\left(\begin{array}{c}
V^{n}\left(a^{k}, \hat{m}^{l}\right)=v^{n} \\
M^{l}=m^{l}, Y^{n}=y^{n}
\end{array}\right) \\
& \leq \sum_{\substack{\hat{a}^{k} \in \mathcal{V}^{k} \\
m^{l}, \hat{m}^{l} \\
m^{l} \neq \hat{m}^{l} \in T_{\eta_{5}(\eta)}(Y)}} \sum_{\substack{y^{n} \\
T_{\eta_{5}(\eta)}\left(V_{1} \oplus V_{2} \mid y^{n}\right)}} P\left(V^{n}\left(a^{k}, \hat{m}^{l}\right)=v^{n}\right) P\left(M^{l}=m^{l}, Y^{n}=y^{n}\right) \\
& \leq \sum_{\hat{a}^{k} \in \mathcal{V}^{k}} \sum_{\hat{m}^{l} \in \mathcal{V}^{l}} \sum_{\substack{y^{n} \\
\in T_{\eta_{5}(\eta)}(Y)}} \sum_{\substack{v^{n} \in \\
\left(V_{1} \oplus V_{2} \mid y^{n}\right)}} \frac{P\left(Y^{n}=y^{n}\right)}{\pi^{n}} \\
& \leq \sum_{\substack{y^{n} \\
\in T_{\eta_{5}(\eta)}(Y)}} \frac{\pi^{k+l}\left|T_{2 \eta_{5}(\eta)}\left(V_{1} \oplus V_{2} \mid y^{n}\right)\right|}{\pi^{n}} \leq \exp \left\{-n \log \pi\left(1-\frac{H\left(V_{1} \oplus V_{2} \mid Y\right)+3 \eta_{5}(\eta)}{\log \pi}-\frac{k+l}{n}\right)\right\}(68)
\end{aligned}
$$

where (68) follows from the uniform bound of $\exp \left\{n\left(H\left(V_{1} \oplus V_{2} \mid Y\right)+3 \eta_{5}(\eta)\right)\right\}$ on $\left|T_{2 \eta_{5}(\eta)}\left(V_{1} \oplus V_{2} \mid y^{n}\right)\right|$ for any $y^{n} \in T_{\eta_{5}(\eta)}(Y), n \geq N_{6}(\eta)$ provided by lemma 5 for $n \geq N_{6}(\eta)$. Substituting the upper bound for $\frac{k+l}{n}$ in 24 , we have

$$
P\left(\epsilon_{5}\right) \leq \exp \left\{-n\left(\eta_{2}(\eta)+\eta_{3}(\eta)-3 \eta_{5}(\eta)\right)\right\} \text { for all } n \geq \max \left\{N_{1}(\eta), N_{6}(\eta)\right\} .
$$

\section{ACKNOWLEDGMENT}

The first author thanks (i) Raj Tejas Suryaprakash for lending his expertise with regard to computing and plotting results for examples 1 - 4, (ii) Aria Sahebi for useful discussions with respect to material presented in section VIII, and (iii) Deepanshu Vasal for general discussions.

\section{REFERENCES}

[1] A. Feinstein, "A new basic theorem of information theory," Information Theory, Transactions of the IRE Professional Group on, vol. 4, no. 4, pp. 2-22, 1954.

[2] I. Csiszar and J. Korner, "Graph decomposition: A new key to coding theorems," Information Theory, IEEE Transactions on, vol. 27, no. 1, pp. 5-12, 1981.

[3] J. Körner and K. Marton, "How to encode the modulo-two sum of binary sources (corresp.)," IEEE Trans. Inform. Theory, vol. 25, no. 2, pp. 219 - 221, Mar 1979.

[4] T. Philosof and R. Zamir, "On the loss of single-letter characterization: The dirty multiple access channel," IEEE Trans. Inform. Theory, vol. 55, pp. 2442-2454, June 2009. 
[5] S. I. Gel'fand and M. S. Pinsker, "Coding for channel with random parameters,” Problems of Ctrl. and Info. Th., vol. 19, no. 1, pp. 19-31, 1980.

[6] R. G. Gallager, Information Theory and Reliable Communication. New York: John Wiley \& Sons, 1968.

[7] D. Slepian and J. Wolf, "Noiseless coding of correlated information sources," Information Theory, IEEE Transactions on, vol. 19, no. 4, pp. 471 - 480, July 1973.

[8] I. Csiszar, "Linear codes for sources and source networks: Error exponents, universal coding," Information Theory, IEEE Transactions on, vol. 28, no. 4, pp. 585-592, 1982.

[9] R. Ahlswede and T. Han, "On source coding with side information via a multiple-access channel and related problems in multi-user information theory,” IEEE Trans. on Info. Th., vol. 29, no. 3, pp. 396 - 412, may 1983.

[10] B. Nazer and M. Gastpar, "Computation over multiple-access channels," IEEE Trans. on Info. Th., vol. 53, no. 10, pp. $3498-3516$, oct. 2007.

[11] A. Padakandla and S. S. Pradhan, "Computing sum of sources over an arbitrary multiple access channel," to appear in proceedings of 2013 IEEE International Symposium on Information Theory Proceedings (ISIT), 2013.

[12] S. Sridharan, A. Jafarian, S. Vishwanath, S. Jafar, and S. Shamai, "A layered lattice coding scheme for a class of three user gaussian interference channels," in 2008 46th Annual Allerton Conference Proceedings on, sept. 2008, pp. 531 -538.

[13] T. Han and K. Kobayashi, "A new achievable rate region for the interference channel," Information Theory, IEEE Transactions on, vol. 27, no. 1, pp. 49 - 60, jan 1981.

[14] A. Padakandla, A. Sahebi, and S. Pradhan, "A new achievable rate region for the 3-user discrete memoryless interference channel," in 2012 IEEE ISIT Proceedings, july 2012, pp. 2256 -2260.

[15] D. Krithivasan and S. Pradhan, "Distributed source coding using abelian group codes: A new achievable rate-distortion region,” IEEE Trans. Inform. Theory, vol. 57, no. 3, pp. 1495 -1519, march 2011.

[16] T. Berger, Multiterminal Source Coding. In: The Information Theory Approach to Communications (ed. G. Longo), CISM Courses and Lecture Notes No. 229. Springer, Wien-New York, 1977.

[17] K. Marton, "A coding theorem for the discrete memoryless broadcast channel," IEEE Trans. Inform. Theory, vol. IT-25, no. 3, pp. 306-311, May 1979.

[18] A. Padakandla and S. Pradhan, "Achievable rate region for three user discrete broadcast channel based on coset codes," available at http://arxiv.org/abs/1207.3146.

[19] R. Sundaresan, "Lecture notes for E2-301 Topics in multi-user communications," Jan-Apr 2008, available at http://ece.iisc.ernet.in/ kprem/e2301.tar.gz.

[20] A. Orlitsky and J. Roche, "Coding for computing," Information Theory, IEEE Transactions on, vol. 47, no. 3, pp. 903 -917, mar 2001.

[21] A. E. Gamal and Y.-H. Kim, Network Information Theory, 1st ed. New York: Cambridge University Press, 2012.

[22] I. Csiszár and J. Körner, Information Theory: Coding Theorems for Discrete Memoryless Systems, 2nd ed. Budapest: Cambridge University Press, June 2011.

[23] T. M. Cover and J. A. Thomas, Elements of Information Theory, 2nd ed. New York: John Wiley \& Sons, 2006.

[24] W. Hoeffding, "Asymptotically optimal tests for multinomial distributions," Annals of Mathematical Statistics, vol. 36, no. 2, pp. 369-401, 1965.

[25] I. Sanov, "On the probability of large deviations of random variables," Matematicheskii Sbornik, vol. 42(84), pp. 11-44, 1957, translated by Dana E. A. Quade, Institute of Statistics, Mimeograph Series No. 192, March 1958, available at. 
[26] R. Barron, B. Chen, and G. W. Wornell, "The duality between information embedding and source coding with side information and some applications," Information Theory, IEEE Transactions on, vol. 49, no. 5, pp. 1159-1180, 2003.

[27] A. Sahebi and S. Pradhan, "Nested lattice codes for arbitrary continuous sources and channels," in Information Theory Proceedings (ISIT), 2012 IEEE International Symposium on, 2012, pp. 626-630.

[28] T. Gariby and U. Erez, "On general lattice quantization noise," in Information Theory, 2008. ISIT 2008. IEEE International Symposium on, 2008, pp. 2717-2721.

[29] R. Ahlswede, "Multi-way communication channels," in Proceedings of 2nd ISIT, Thakadsor, Armenian SSR, Sept 1971, pp. 23-52.

[30] H. Liao, "A coding theorem for multiple access communications," in Proceedings of 2nd ISIT, Thakadsor, Armenian SSR, 1972.

[31] E. Haim, Y. Kochman, and U. Erez, "Expurgation for discrete multiple-access channels via linear codes," in Information Theory Proceedings (ISIT), 2012 IEEE International Symposium on, 2012, pp. 31-35.

[32] A. Padakandla and S. Pradhan, "Achievable rate region based on coset codes for multiple access channel with states," available at http://arxiv.org/abs/1301.5655.

[33] M. Gastpar, B. Rimoldi, and M. Vetterli, “To code, or not to code: Lossy source-channel communication revisited," IEEE Trans. Inform. Theory, vol. 49, no. 3, pp. 1147-1158, May 2003.

[34] S. Pradhan and K. Ramchandran, "On functional duality in multiuser source and channel coding problems with one-sided collaboration," Information Theory, IEEE Transactions on, vol. 52, no. 7, pp. 2986-3002, 2006.

[35] S. I. Gelfand, "Capacity of one broadcast channel,” Probl. Pered. Inform., vol. 13, no. 3, pp. 106108, JulySept. 1977; translated in Probl. Inform. Transm., pp. 240242, JulySept. 1977.

[36] A. Sahebi and S. Pradhan, "On distributed source coding using Abelian group codes," in Proceedings of 2012 50th Annual Allerton Conference on Communication, Control, and Computing (Allerton), oct. 2012.

[37] R. Ahlswede and J. Gemma, "Bounds on algebraic code capacities for noisy channels. I," Information and Control, vol. 19, no. 2, pp. 124 - 145, 1971. [Online]. Available: http://www.sciencedirect.com/science/article/pii/S0019995871907753

[38] — - "Bounds on algebraic code capacities for noisy channels. II," Information and Control, vol. 19, no. 2, pp. 146 158, 1971. [Online]. Available: http://www.sciencedirect.com/science/article/pii/S0019995871907832

[39] R. Ahlswede, "Group codes do not achieve shannon's channel capacity for general discrete channels," The Annals of Mathematical Statistics, vol. 42, no. 1, pp. 224-240, February 1971.

[40] A. Sahebi and S. Pradhan, "Abelian group codes for source coding and channel coding," submitted to IEEE Trans. of Information theory, April 2013, available at http://arxiv.org/abs/1305.1598.

[41] A. El Gamal and E. Van der Meulen, "A proof of Marton's coding theorem for the discrete memoryless broadcast channel," IEEE Trans. Inform. Theory, vol. IT-27, no. 1, pp. 120-122, Jan. 1981. 